\title{
Reflexão de funções cardinais
}

\author{
Alberto Marcelino Efigênio Levi
}

\author{
TESE APRESENTADA \\ $\mathrm{AO}$ \\ Instituto DE Matemática e Estatística \\ DA \\ Universidade de SÃo Paulo \\ PARA \\ OBTENÇÃO DO TÍTULO \\ $\mathrm{DE}$ \\ Doutor EM CIÊNCIAS
}

Programa: Matemática
Orientadora: Profa. Dra. Lúcia Renato Junqueira

Durante o desenvolvimento deste trabalho o autor recebeu auxílio financeiro da CAPES e do CNPq

São Paulo, agosto de 2012 



\section{Reflexão de funções cardinais}

Este exemplar corresponde à redação final da tese devidamente corrigida e defendida por Alberto Marcelino Efigênio Levi e aprovada pela Comissão Julgadora.

Banca Examinadora:

- Profa. Dra. Lúcia Renato Junqueira (orientadora) - IME-USP.

- Prof. Dr. Ricardo Bianconi - IME-USP.

- Prof. Dr. Marcelo Coniglio - UNICAMP.

- Prof. Dr. Marcelo Dias Passos - UFBA.

- Prof. Dr. Leandro Fiorini Aurichi - ICMC-USP. 



\section{Agradecimentos}

São muitas as pessoas, que de uma forma ou de outra, me apoiaram nestes anos de estudos: familiares, grandes amigos, além de professores e colegas, tanto no Bacharelado na UFPR quanto no Doutorado na USP. Agradeço particularmente à Profa. Ofélia Alas, pelos valorosos conselhos; à Profa. Lúcia Junqueira, por ter me orientado no Doutorado, sempre de uma forma muito presente e atenciosa, e também com discernimento e objetividade que considero admiráveis; e à minha mãe, Maria do Rocio, pela convivência e apoio inestimáveis ao longo destes anos. 


\section{Resumo}

Neste trabalho investigamos problemas sobre reflexão de funções cardinais, fazendo uso de técnicas como submodelos elementares e Teoria PCF. Mostramos que o grau de Lindelöf reflete todos os cardinais fortemente inacessíveis e que um exemplo de espaço onde a mesma função cardinal não reflita um cardinal fracamente inacessível requer a existência de $0^{\sharp}$. Além disso, estendemos um resultado de reflexão do caráter, de espaços Lindelöf para espaços linearmente Lindelöf, obtendo novas equivalências com a Hipótese do Contínuo $(\mathrm{CH})$. Obtivemos ainda várias respostas parciais para problemas clássicos deste tópico de pesquisa. 


\section{Abstract}

This work investigates problems about reflection of cardinal functions, using techniques such as elementary submodels and PCF Theory. We show that the Lindelöf degree reflects all the strongly inaccessible cardinals and that a example of a space in which the same cardinal function does not reflect a weakly inaccessible cardinal requires " $0^{\sharp}$ exists". Furthermore, we extend a result of reflection of the character from Lindelöf spaces to linearly Lindelöf spaces, obtaining new equivalences with the Continuum Hypothesis $(\mathrm{CH})$. We also obtained several partial answers to classic problems of this research topic. 


\section{Sumário}

Introdução $\quad$ xiii

1 Preliminares 1

1.1 Teoria dos conjuntos . . . . . . . . . . . . . . 1

1.2 Topologia Geral . . . . . . . . . . . . . . . . . . . . 6

1.3 Submodelos elementares . . . . . . . . . . . . . . . 15

1.4 Reflexão de funções cardinais . . . . . . . . . . . . . . . . . . . 17

2 Reflexões em funções locais $\quad 19$

2.1 Introdução . . . . . . . . . . . . . . . . . . . . 19

2.2 Espaços diádicos . . . . . . . . . . . . . . . . . . . 20

2.3 Espectros de caráter e convergência . . . . . . . . . . . . . . 24

2.4 Classificação dos possíveis contra-exemplos . . . . . . . . . . . . . 28

2.5 Resultados com submodelos elementares . . . . . . . . . . . . . 31

3 CH e reflexão do caráter $\quad 35$

3.1 Introdução . . . . . . . . . . . . . . . . . . . . 35

3.2 Resultados auxiliares . . . . . . . . . . . . . . . . 36

3.3 Reflexão do caráter . . . . . . . . . . . . . . . . . . . . . . . 40

4 Funções globais $\quad 47$

4.1 Introdução . . . . . . . . . . . . . . . . . . . . . . . . . . . . 47

4.2 Resultados relacionados ao spread . . . . . . . . . . . . 48

4.3 L e cardinais fortemente inacessíveis . . . . . . . . . . . . . . . . 50

4.4 Reflexão em cardinais fracamente inacessíveis . . . . . . . . . . . 55

4.5 Reflexão de ll . . . . . . . . . . . . . . . . . . . . . 63

A Resultados combinatórios $\quad \mathbf{6 5}$

A.1 Propriedades gerais . . . . . . . . . . . . . . . . 65

A.2 Propriedades de SSH $\ldots \ldots \ldots$. . . . . . . . . . . . 68

$\begin{array}{ll}\text { Referências Bibliográficas } & 71\end{array}$ 


\section{Introdução}

O conceito de reflexão que estudaremos neste trabalho é similar ao encontrado em outros contextos da Matemática. Por exemplo, em Teoria dos Conjuntos existem os "Princípios de Reflexão" (ver [23] ou [34]), onde dada uma lista finita de sentenças válidas no universo dos conjuntos, há um conjunto (um modelo) onde estas mesmas sentenças permanecem válidas.

Uma idéia semelhante existe em Topologia Geral. Neste caso, o conceito de reflexão é de que se alguma propriedade $P$ é verificada em um espaço topológico $X$, também é verificada em algum de seus subespaços "pequenos". Podemos reformular este conceito, se considerarmos mais conveniente, substituindo $P$ pela propriedade complementar $Q=\neg P$, e então teremos que $X$ satisfaz $Q$ desde que todos os seus subespaços "pequenos" satisfaçam $Q$.

Existem diferentes noções de reflexões topológicas, cada uma associada a um conceito diferente de subespaço "pequeno". Considere os seguintes resultados:

Teorema (Tkachuk, [44]). Seja X um espaço $T_{2}$ tal que $\bar{D}$ é compacto para todo $D \subset X$ discreto. Então $X$ é compacto.

Teorema (Hajnal e Juhász, [20]). Sejam $X$ um espaço topológico e $\kappa$ um cardinal não enumerável. Se todo subespaço $Y$ de $X$ com $|Y| \leq \kappa$ admitir uma base de abertos de cardinalidade menor que $\kappa$, então $X$ admite uma base de abertos de cardinalidade menor que $\kappa$.

Estes dois resultados diferem entre si, tanto pela propriedade que está sendo "refletida", quanto pelo conceito adotado de subespaço "pequeno". No Teorema de Tkachuk acima, a propriedade que está sendo refletida é o de ser um espaço compacto, e os subespaços considerados pequenos são os fechos de subespaços discretos.

Por outro lado, o Teorema de Hajnal e Juhász, citado acima, é um exemplo do que chamamos de teoremas de reflexão para funções cardinais. Este tipo de resultado de reflexão constitui o escopo do presente trabalho. Em resultados de 
reflexão desta natureza, a propriedade refletida está relacionada ao valor que alguma função cardinal assume em um espaço topológico, e os subespaços pequenos são aqueles de cardinalidade limitada por um determinado cardinal fixado. Mais precisamente, adotamos a seguinte definição:

Definição (Hodel e Vaughan, [22]). Sejam $\phi$ uma função cardinal, $\kappa$ um cardinal infinito e $S$ uma classe de espaços topológicos. Então " $\phi$ reflete $\kappa$ para $S^{\prime \prime}$ significa:

se $X \in S$ e $\phi(X) \geq \kappa$, então existe $Y \subset X$ tal que $|Y| \leq \kappa$ e $\phi(Y) \geq \kappa$.

Ou de forma equivalente:

se $X \in S$ e $\phi(Y)<\kappa$ para todo $Y \subset X$ com $|Y| \leq \kappa$, então $\phi(X)<\kappa$.

Quando $S$ for a classe de todos os espaços topológicos, podemos dizer simplesmente " $\phi$ reflete $\kappa "$.

De acordo com esta definição, o Teorema de Hajnal e Juhász pode ser reescrito como: "dado qualquer cardinal não-enumerável $\kappa, w$ reflete $\kappa$ ", onde $w$ é a função cardinal peso, definida como sendo a cardinalidade mínima de uma base do espaço. Iremos seguir neste trabalho a convenção estabelecida em [21] e em outros textos de referência, de que toda função cardinal assume apenas valores infinitos. Com esta consideração, é imediato que qualquer função cardinal reflete $\omega$, e portanto $w$ reflete todos os cardinais infinitos.

Como veremos neste trabalho, há outras funções cardinais que se comportam como o peso, ou seja, refletem todos os cardinais infinitos. No entanto, isto não ocorrerá com todas. Para outras funções cardinais, a reflexão se verificará ou não dependendo da classe de espaços considerada, ou do tipo de cardinal infinito, como ilustram os seguintes resultados, onde $\chi$ e $d$ são, respectivamente, as funções cardinais caráter e densidade (ver [22]):

Teorema. Seja $\kappa$ um cardinal não enumerável. Existe um espaço $T_{2}$, paracompacto e completamente normal $X$ tal que $\chi(X)=\kappa^{+}$mas $\chi(Y)<\kappa$ para todo $Y \subset X$ com $|Y| \leq \kappa$. Este espaço é portanto um contra-exemplo para o enunciado " $\chi$ reflete todos os cardinais infinitos".

Teorema. $\chi$ reflete todos os cardinais infinitos para a classe dos espaços compactos $T_{2}$.

Teorema. $d$ reflete todos os cardinais regulares. 
Teorema. Existe um contra-exemplo para o enunciado "d reflete todos os cardinais singulares para a classe dos espaços $T_{1}{ }^{\prime \prime}$.

Os primeiros estudos sobre reflexão de funções cardinais e conceitos relacionados foram realizados por Tkačenko [43] e Juhász [24]. Um marco importante neste tópico foi o Teorema de Hajnal e Juhász de reflexão do peso [20]. Posteriormente, outra contribuição importante foi o Teorema de Dow de reflexão da metrizabilidade [11][10], não apenas pelo resultado, mas pela introdução da técnica de submodelos elementares em problemas de Topologia Geral.

O artigo de Hodel e Vaughan [22] foi o primeiro estudo sistemático de reflexão de funções cardinais. Nele, são apresentadas definições relacionadas ao tópico e os principais resultados até então conhecidos sobre reflexão de funções cardinais, além de resultados novos. Este artigo salienta que vários problemas permaneceram em aberto. Alguns artigos posteriores, como [7], procuraram fornecer respostas completas ou parciais a algumas destas questões. Uma outra referência importante para este trabalho foi a Dissertação de Mestrado de Rodrigo R. Dias [9], sob o título "Reflexão de funções cardinais e da metrizabilidade".

Neste trabalho, investigamos alguns dos principais problemas relativos a reflexão de funções cardinais, e apresentamos algumas respostas, completas ou parciais. Alguns dos problemas aqui considerados podem também ser situados no contexto de outros tópicos de pesquisa da Topologia Geral - como os problemas sobre espaços linearmente Lindelöf (ver [39]). No desenvolvimento deste trabalho, fizemos uso de várias ferramentas que hoje são importantes ou tem ganhado importância em Topologia Geral - como os submodelos elementares e a Teoria PCF de Shelah.

Esta tese é composta de quatro capítulos e um apêndice. No capítulo 1, apresentamos os conceitos, notações e resultados utilizados ao longo do trabalho, relativos à Teoria dos Conjuntos, Topologia Geral (incluindo todas as funções cardinais estudadas neste trabalho), submodelos elementares e reflexão de funções cardinais. O objetivo deste capítulo é facilitar a leitura dos capítulos posteriores, evitando excessivas consultas a outras fontes.

O capítulo 2 é dedicado a estudar reflexão de funções cardinais locais, como caráter $(\chi)$ e pseudocaráter $(\psi)$. Sob $\mathrm{GCH}, \psi$ reflete todos os cardinais infinitos para a classe dos espaços compactos $T_{2}$, e um dos principais problemas em aberto neste tópico é se podemos mostrar o mesmo resultado sem assumir GCH. Uma resposta parcial foi fornecida por [7]: pode-se mostrar em ZFC que $\psi$ reflete todos os cardinais infinitos para a classe dos compactos diádicos. Nós apresentamos uma 
demonstração mais simples para o mesmo resultado, além de obter outros resultados parciais, que ampliam a classe de espaços na qual se verifica este resultado de reflexão. Ao fazer isso, encontramos conexões deste problema com problemas em aberto de outros tópicos de pesquisa em Topologia Geral. Muitos dos resultados que obtivemos para $\psi$ são válidos também para a função cardinal psw (point separating weight), que apresenta comportamento semelhante com relação à reflexão. Ainda no capítulo 2, obtemos mais alguns resultados de reflexão para $\chi$ e $\psi$ usando submodelos elementares.

O estudo de funções cardinais locais prossegue no capítulo 3. Dado um cardinal infinito $\kappa, \chi$ reflete $\kappa^{+}$para a classe dos espaços compactos $T_{2}$. Quando se considera ampliar a classe de espaços, de "compactos $T_{2}$ " para "espaços Lindelöf $T_{2}$ ", encontra-se uma inesperada equivalência entre um enunciado topólogico e outro puramente conjuntista: " $2^{\kappa}=\kappa^{+}$" é equivalente em ZFC a " $\chi$ reflete $\kappa^{+}$para a classe dos espaços Lindelöf $T_{2}$ " (ver [26] ou [5]). Podemos considerar uma ampliação ainda maior na classe de espaços, por exemplo, de "espaços Lindelöf $T_{2}$ " para "espaços linearmente Lindelöf $T_{2}$ ", e investigar o que acontece. Tal questionamento se insere no contexto de problemas sobre espaços linearmente Lindelöf, onde se investiga quais propriedades dos espaços Lindelöf são ainda válidas na classe dos espaços linearmente Lindelöf. Os resultados que obtivemos nos permitem enunciar novas equivalências topológicas com a hipótese " $2^{\kappa}=\kappa^{+}$", a qual corresponde à Hipótese do Contínuo $(\mathrm{CH})$ quando $\kappa=\omega$.

No capítulo 4 estudamos a reflexão de funções cardinais globais, como a densidade $(d)$ e o grau de Lindelöf $(L)$. Hajnal e Juhász mostraram em [19] que $d$ reflete todo cardinal regular e todo cardinal de cofinalidade enumerável para a classe dos espaços $T_{2}$, e perguntaram se o mesmo ocorre nos cardinais singulares de cofinalidade não-enumerável. Obtivemos respostas parciais, para as classes dos espaços compactos $T_{2}$ e dos espaços $d$-separáveis. Quanto ao grau de Lindelöf, um dos resultados em [22] estabelece que:

Teorema. ( $G C H+$ não existem cardinais inacessiveis) L reflete todos os cardinais para a classe dos espaços $T_{2}$.

Nós investigamos se a hipótese de não existir cardinais inacessíveis era realmente necessária. Usando submodelos elementares, e propondo uma definição alternativa (mas equivalente) para o grau de Lindelöf, mostramos que $L$ reflete todos os cardinais fortemente inacessíveis, e portanto a hipótese referida acima pode ser retirada do enunciado do teorema. Analisamos ainda a questão: $L$ reflete os cardinais fracamente inacessíveis? Obtivemos algumas respostas parciais, utilizando conceitos combinatórios de aritmética cardinal e Teoria PCF, e estabelecemos que um eventual contra-exemplo a essa questão requer algum axioma 
de grandes cardinais pelo menos tão forte quanto "existe $0^{\sharp " . ~ E s t u d a m o s ~ a i n d a ~}$ a função cardinal $l l$ (grau de Lindelöf linear), fazendo uma análise semelhante à realizada para $L$.

Vários resultados puramente combinatórios necessários ao capítulo 4 foram separados em um apêndice, mantendo-se no capítulo 4 os resultados principais sobre reflexão.

Os resultados que são apresentados sem autoria mencionada, são do autor. 


\section{Capítulo 1}

\section{Preliminares}

\subsection{Teoria dos conjuntos}

Neste trabalho a teoria de conjuntos adotada será ZFC (Zermelo-Fraenkel mais o Axioma da Escolha). Nossas principais referências serão [23] e [34], nas quais se encontram as definições dos conceitos básicos, como boa ordem, ordinais, tipo de ordem, cardinais e cofinalidades, bem como as notações, definições e resultados que apresentaremos nesta seção, salvo indicação em contrário. Usualmente nomearemos ordinais com as letras $\alpha, \beta, \gamma$ e $\delta$, e cardinais com as letras $\kappa, \lambda$ e $\mu$. Com frequência expressaremos um cardinal $\aleph_{\alpha} \operatorname{como} \omega_{\alpha}$, e $\aleph_{0} \operatorname{como} \omega=\omega_{0}$. Dado um conjunto $X$, denotaremos por $|X|$ sua cardinalidade, e por $\mathcal{P}(X)$ o conjunto de todos os seus subconjuntos.

A cofinalidade de um ordinal $\alpha$, que denotaremos por $c f(\alpha)$, é o menor ordinal limite $\beta$ tal que exista uma sequência crescente $\left\{\alpha_{\xi}: \xi<\beta\right\}$ de ordinais com $\lim _{\xi \rightarrow \beta} \alpha_{\xi}=\alpha$. Se $(W, \leq)$ é um conjunto bem ordenado, denotaremos por ot $(W, \leq)$ ou ot $(W)$ seu tipo de ordem (order-type), que é o ordinal isomorfo a $(W, \leq)$. Um cardinal $\kappa$ é regular se $c f(\kappa)=\kappa$ e singular se $c f(\kappa)<\kappa$. Para qualquer ordinal $\alpha, c f(\alpha)$ é sempre um cardinal regular. Dado qualquer cardinal $\kappa, \kappa^{+}$denotará o cardinal sucessor de $\kappa$, e $2^{\kappa}$ a cardinalidade de $\mathcal{P}(\kappa)$. Um cardinal $\kappa>\omega$ é limite, se $\lambda^{+}<\kappa$ para todo $\lambda<\kappa$, e é limite forte se $2^{\lambda}<\kappa$ para todo $\lambda<\kappa$. Todo cardinal sucessor é regular. Denotaremos por ORD a classe de todos os ordinais, CARD a classe de todos os cardinais infinitos, e por REG a classe de todos os cardinais infinitos regulares. Quando $S$ for uma classe própria e escrevermos $X \in S$, isto deve ser entendido como $X$ satisfazendo uma fórmula que define a classe $S$.

Definição 1.1.1 Sejam $\lambda$ e $\mu$ cardinais. 
- $\lambda+\mu=|X \cup Y|$, onde $X$ e $Y$ são conjuntos com $X \cap Y=\emptyset,|X|=\lambda e$ $|Y|=\mu$

- $\lambda \cdot \mu=|\lambda \times \mu|$.

Proposição 1.1.2 Dados cardinais infinitos $\lambda e \mu$,

$$
\lambda+\mu=\lambda \cdot \mu=\max \{\lambda, \mu\} .
$$

Definição 1.1.3 Dada uma família $\left\{\kappa_{i}: i \in I\right\}$ de cardinais, definimos

$$
\sum_{i \in I} \kappa_{i}=\left|\bigcup_{i \in I} X_{i}\right|,
$$

onde $\left\{X_{i}: i \in I\right\}$ é uma família disjunta de conjuntos, com $\left|X_{i}\right|=\kappa_{i}$ para todo $i \in I$.

Definição 1.1.4 Dados cardinais $\kappa$ e $\lambda, \kappa^{\lambda}$ será a cardinalidade do conjunto de todas as funções de $\lambda \mathrm{em} \kappa$. Se $\lambda$ for um cardinal limite, definimos

$$
\kappa^{<\lambda}=\sup \left\{\kappa^{\mu}: \mu \text { é cardinal e } \mu<\lambda\right\} .
$$

Definição 1.1.5 A função beth $^{1} \beth: O R D \rightarrow C A R D$ é definida por indução transfinita:

- $\beth_{0}=\aleph_{0}$;

- $\beth_{\alpha+1}=2^{\beth_{\alpha}}$;

- se $\alpha$ for um ordinal limite, $\beth_{\alpha}=\sup \left\{\beth_{\beta}: \beta<\alpha\right\}$.

Definição 1.1.6 Dado um conjunto $X$ e um cardinal $\kappa$, definimos:

\footnotetext{
${ }^{1}$ Não é propriamente uma função, pois seu "domínio" é a classe de todos os ordinais, e portanto, uma classe própria. A mesma observação valerá para outras "funções" que definiremos em classes próprias, como as funções potência e gimel.
} 
- $[X]^{\kappa}=\{Y \subset X:|Y|=\kappa\}$;

- $[X]^{<\kappa}=\{Y \subset X:|Y|<\kappa\}$;

- $[X]^{\leq \kappa}=\{Y \subset X:|Y| \leq \kappa\}$.

Proposição 1.1.7 Dados cardinais infinitos $\kappa \geq \lambda,\left|[\kappa]^{\lambda}\right|=\kappa^{\lambda}$.

Definição 1.1.8 Se $\lambda$ e $\mu$ são cardinais com $\lambda \leq \mu$, definimos os seguintes intervalos de cardinais:

- $[\lambda, \mu]=\{\kappa: \kappa$ é cardinal e $\lambda \leq \kappa \leq \mu\}$;

- $[\lambda, \mu[=\{\kappa: \kappa$ é cardinal e $\lambda \leq \kappa<\mu\}$;

- $] \lambda, \mu]=\{\kappa: \kappa$ é cardinal e $\lambda<\kappa \leq \mu\}$;

- $] \lambda, \mu[=\{\kappa: \kappa$ é cardinal e $\lambda<\kappa<\mu\}$.

Teorema 1.1.9 A função potência que leva cada cardinal $\kappa$ em $2^{\kappa}$ satisfaz as seguintes propriedades:

1. se $\kappa<\lambda$ então $2^{\kappa} \leq 2^{\lambda}$;

2. $c f\left(2^{\kappa}\right)>\kappa$.

Definição 1.1.10 A função gimel I : CARD $\rightarrow$ CARD é a que leva cada cardinal $\kappa$ em $\beth(\kappa)=\kappa^{c f(\kappa)}$.

Teorema 1.1.11 A função potência pode ser definida em termos da função gimel:

1. se $\kappa$ for um cardinal regular, $2^{\kappa}=\beth(\kappa)$;

2. se $\kappa$ for um cardinal limite, e a função potência abaixo de $\kappa$ for eventualmente constante (ou seja, se existir um cardinal $\lambda<\kappa$ tal que $2^{\mu}=2^{\lambda}$ sempre que $\lambda \leq \mu<\kappa)$, então $\left.2^{\kappa}=2^{<\kappa} \cdot\right](\kappa) ;$ 
3. se $\kappa$ for um cardinal limite, e a função potência abaixo de $\kappa$ não for eventualmente constante, então $2^{\kappa}=\Xi\left(2^{<\kappa}\right)$.

O próximo teorema é devido a Easton:

Teorema 1.1.12 Seja uma função $F: R E G \rightarrow C A R D$ satisfazendo as seguintes propriedades:

1. se $\kappa<\lambda$ então $F(\kappa) \leq F(\lambda)$;

2. $c f(F(\kappa))>\kappa$ para todo $\kappa$ regular.

Então, é consistente com ZFC que tenhamos $2^{\kappa}=F(\kappa)$ para todo cardinal regular $\kappa$.

Em contraste com esta grande "liberdade" com que se pode fixar as potências de cardinais regulares, as potências de cardinais singulares estão sujeitas à restrições mais severas, como as expressas pelos seguintes teoremas:

Teorema 1.1.13 (Silver) Seja $\kappa$ um cardinal singular com $\mathrm{cf}(\kappa)>\omega$. Se $2^{\lambda}=$ $\lambda^{+}$para todo cardinal $\lambda<\kappa$, então $2^{\kappa}=\kappa^{+}$.

Teorema 1.1.14 [1] (Galvin-Hajnal, Shelah para cofinalidade enumerável) Se $\kappa=$ $\aleph_{\alpha}$ for um cardinal singular e limite forte, então

$$
2^{\kappa}<\aleph_{\left(2^{|\alpha|}\right)^{+}}
$$

Teorema 1.1.15 [1] (Shelah) Se $\kappa=\aleph_{\alpha}<\aleph_{\kappa}$ for um cardinal singular e limite forte, então

$$
2^{\kappa}<\aleph_{|\delta|^{++++}}
$$

Denotaremos por FIX a classe de todos os cardinais que são pontos fixos da função aleph, ou seja, os cardinais $\kappa$ tais que $\aleph_{\kappa}=\kappa$. Note que o último teorema acima se aplica apenas a cardinais que não sejam pontos fixos. Dado qualquer cardinal $\kappa$, há pontos fixos maiores que $\kappa$ e o menor destes é

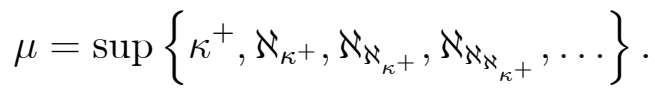


Definição 1.1.16 A Hipótese do Contínuo (CH) é a afirmação "2 $2^{\aleph_{0}}=\aleph_{1}{ }^{\prime \prime}$, e a Hipótese do Continuo Generalizada (GCH) é a afirmação $" 2^{\kappa}=\kappa^{+}$para todo cardinal infinito $\kappa "$.

Definição 1.1.17 A Hipótese para Cardinais Singulares (SCH) é a afirmação "para cada cardinal singular $\kappa$, se $2^{c f(\kappa)}<\kappa$ então $\beth(\kappa)=\kappa^{+}$".

Tanto CH quanto GCH são independentes de ZFC. Sob GCH, todo cardinal limite é limite forte. É fácil ver que $\mathrm{GCH}$ implica $\mathrm{SCH}$, logo $\mathrm{SCH}$ é consistente com ZFC.

Ao longo deste trabalho, quando se colocar no início do enunciado de um teorema uma hipótese como $(\mathrm{CH})$ ou $(\mathrm{GCH})$, isso significa que o restante do enunciado é teorema na Teoria de Conjuntos "ZFC + Hipótese".

Definição 1.1.18 Um cardinal fracamente inacessível é um cardinal limite e regular. Um cardinal fortemente inacessivel é um cardinal limite forte e regular.

Todo cardinal fracamente inacessível é um ponto fixo da função aleph. É consistente com ZFC que não existam cardinais fracamente inacessíveis, mas não se pode demonstrar que a existência destes cardinais seja consistente com ZFC. Outro tipo de "grande cardinal" que aparecerá neste trabalho são os cardinais fracamente compactos (weakly compact cardinals). Se $\kappa$ for fracamente compacto, então $\kappa$ é fortemente inacessível, e há $\kappa$ cardinais fortemente inacessíveis menores que $\kappa$. Portanto, o primeiro cardinal fortemente inacessível não é fracamente compacto.

Um outro axioma de grandes cardinais que citaremos é "existe $0^{\sharp} "$. $0^{\sharp}$ (zero sharp) não é um cardinal (ver [23] ou [32]), mas o enunciado "existe $0^{\sharp " ~ i m p l i c a ~}$ a existência de alguns tipos de grandes cardinais, inclusive cardinais fracamente compactos. Se a Teoria "ZFC + existe um cardinal fortemente inacessível" for consistente, também será a Teoria "ZFC + existe um cardinal fortemente inacessível $+0^{\sharp}$ não existe".

A negação de SCH é equiconsistente com a existência de um cardinal mensurável $\kappa$ de Ordem de Mitchell $\kappa^{++}$(ver [23]). A existência de um cardinal mensurável implica a existência de $0^{\sharp}$ (ver [32]), e portanto "não existe $0^{\sharp}$ " implica $\mathrm{SCH}$. 
Definição 1.1.19 Uma árvore é um conjunto parcialmente ordenado $(T,<)$ tal que, para todo $x \in T$, o conjunto $\{y \in T: y<x\}$ é bem ordenado por $<$. Para cada $x \in T$, definimos $l(x)$ como o ordinal isomorfo a $\{y \in T: y<x\}$. Um subconjunto $S$ de $T$ é um ramo em $T$ se for uma cadeia maximal. Uma raiz de $T$ é um elemento minimal em $T$. Dados $x, y \in T$, y é sucessor de $x$ se $x<y e$ $l(y)=l(x)+1$. Uma folha de $T$ é um elemento maximal em $T$.

\subsection{Topologia Geral}

Nossas principais referências serão [14] para os conceitos básicos de Topologia Geral e [21] para definições de várias funções cardinais, bem como algumas de suas propriedades.

Definição 1.2.1 [19] Um espaço $X$ é separado à direita se existe uma boa ordenação $\left\{x_{\alpha}: \alpha<\delta\right\}$ de $X$ e uma sequência $\left\{U_{\alpha}: \alpha<\delta\right\}$ de abertos de $X$ tais que $x_{\alpha} \in U_{\alpha}$ e $x_{\beta} \notin U_{\alpha}$ sempre que $\alpha<\beta<\delta$. Um espaço $X$ separado $\grave{a}$ esquerda é definido de forma análoga, com $x_{\alpha} \in U_{\alpha}$ e $x_{\beta} \notin U_{\alpha}$ sempre que $\beta<\alpha<\delta$.

Definição 1.2.2 Um espaço $X$ é compacto, se toda cobertura aberta tiver alguma subcobertura finita.

Na definição acima não assumimos nenhum axioma de separação.

Definição 1.2.3 [18] Um compactum (compacta no plural) é um espaço compacto $T_{2}$ infinito.

Definição 1.2.4 Dado um subconjunto $A$ de um espaço topológico $X$, dizemos que $x \in X$ é um ponto de acumulação completo de $A$ se $|A \cap U|=|A|$ para toda vizinhança aberta $U$ de $x$.

Um espaço $T_{2}$ é compacto se e somente se todo conjunto infinito tiver ponto de acumulação completo. 
Definição 1.2.5 Dado um cardinal infinito $\kappa$, o Cubo de Cantor $2^{\kappa}$ é o produto $\prod_{\alpha \in \kappa} D_{\alpha}$, munido da topologia produto, onde cada $D_{\alpha}$ é o espaço discreto $2=$ $\{0,1\}$. Para cada $\delta \in \kappa$, definimos a projeção $\pi_{\delta}: \prod_{\alpha \in \kappa} D_{\alpha} \rightarrow D_{\delta}$, e para cada $S \subset \kappa$, a projeção $\pi_{S}: \prod_{\alpha \in \kappa} D_{\alpha} \rightarrow \prod_{\alpha \in S} D_{\alpha}$.

Pelo Teorema de Tychonoff, o Cubo de Cantor é um espaço compacto $T_{2}$. Quando $\kappa=\omega$, chamamos o Cubo de Cantor $2^{\omega}$ de Conjunto de Cantor, por ser homeomorfo ao Conjunto de Cantor usualmente definido no intervalo $[0,1]$ da reta real.

Definição 1.2.6 Se $X$ for um espaço topológico, $Y$ um espaço compacto $T_{2}$, e $c: X \rightarrow Y$ um homeomorfismo entre $X$ e $c[X]$, com $\overline{c[X]}=Y$, dizemos que o par $(Y, c)$ é uma compactificação de $X$.

Um espaço $X$ possui alguma compactificação se e somente se for um espaço Tychonoff.

Definição 1.2.7 Se X for um espaço Tychonoff, localmente compacto mas não compacto, definimos a compactificação de Alexandroff da seguinte forma: escolha um ponto $p \notin X$ e defina $Y=X \cup\{p\}$, munido da seguinte topologia: os subespaços abertos de $X$ serão abertos em $Y$ e as vizinhanças abertas de $p$ serão exatamente os conjuntos $Y \backslash K$, para os subespaços compactos $K \subset X$.

Definição 1.2.8 Dado um espaço Tychonoff $X$, a compactificação de StoneČech de $X$, denotada por $\beta X$, é a compactificação de $X$ tal que toda função continua $f: X \rightarrow[0,1]$ pode ser estendida continuamente à uma função $F$ : $\beta X \rightarrow[0,1]$.

Definição 1.2.9 Um espaço diádico é um compacto $T_{2}$ que seja imagem contínua de algum Cubo de Cantor $2^{\kappa}$.

Considerando o ordinal $\omega$ munido da topologia discreta, $\beta \omega$ é um exemplo de espaço compacto $T_{2}$ que não é diádico. 
Definição 1.2.10 [42] Dados cardinais infinitos $\lambda$ e $\kappa$, com $\lambda \leq \kappa$, um espaço $X$ é $[\lambda, \kappa]$-compacto se toda cobertura aberta de cardinalidade $\leq \kappa$ tiver alguma subcobertura de cardinalidade $<\lambda$. Se $\lambda=\omega$, dizemos que o espaço é inicialmente $\kappa$-compacto, e se $\kappa \geq|X|$, dizemos que o espaço é finalmente $\lambda$-compacto.

Os espaços Lindelöf são exatamente os espaços finalmente $\omega_{1}$-compactos. Uma terminologia que aparece em [4], [6] e [39] é o de espaços $\omega_{1}$-Lindelöf, ou mais geralmente, $\kappa$-Lindelöf, que são os espaços $\left[\omega_{1}, \kappa\right]$-compactos. Outra notação que usaremos em alguns momentos, quando $\kappa$ for um cardinal regular, é o de $L(\kappa)$ espaço, que corresponde a um espaço $[\kappa, \kappa]$-compacto.

Definição 1.2.11 [39] Um espaço X é linearmente Lindelöf, se toda cobertura aberta, linearmente ordenada pela inclusão, tiver alguma subcobertura enumerável.

Caracterizações equivalentes dessa propriedade serão apresentadas de forma generalizada após definirmos a função cardinal $l l$. Todo espaço Lindelöf é linearmente Lindelöf, mas a recíproca não é verdadeira, alguns dos contra-exemplos conhecidos estão descritos em [39], [35] e [15].

Definição 1.2.12 Uma função cardinal é uma função $\phi: T \rightarrow C A R D$, onde $T$ é uma classe de espaços topológicos (usualmente a classe de todos os espaços topológicos), tal que $\phi(X)=\phi(Y)$ sempre que $X$ e $Y$ sejam espaços homeomorfos.

Apresentaremos agora as definições das funções cardinais utilizadas neste trabalho (ver [21]). Em todas as definições a seguir, $X$ é um espaço topológico.

Definição 1.2.13 O peso de $X$ é definido como:

$$
w(X)=\min \{|\mathcal{B}|: \mathcal{B} \text { é uma base de } X\}+\omega .
$$

Definição 1.2.14 A densidade de $X$ é definida como:

$$
d(X)=\min \{|S|: S \subset X, \bar{S}=X\}+\omega .
$$


Definição 1.2.15 A celularidade de X é definida como:

$$
c(X)=\sup \{|\mathcal{V}|: \mathcal{V} \text { é uma família celular em } X\}+\omega ;
$$

uma família celular é uma família de abertos de $X$, dois a dois disjuntos.

Definição 1.2.16 O spread de $X$ é definido como:

$$
s(X)=\sup \{|D|: D \subset X, D \text { é discreto }\}+\omega .
$$

Definição 1.2.17 $O$ extent de $X$ é definido como:

$$
e(X)=\sup \{|D|: D \subset X, D \text { é fechado e discreto }\}+\omega .
$$

Definição 1.2.18 O freeness de $X$ é definido como:

$$
F(X)=\sup \{\kappa: X \text { tem uma sequência livre de comprimento } \kappa\}+\omega ;
$$

uma sequência livre de comprimento $\kappa$, é uma sequência $\left\{x_{\alpha}: \alpha<\kappa\right\}$ tal que

$$
\overline{\left\{x_{\alpha}: \alpha<\beta\right\}} \cap \overline{\left\{x_{\alpha}: \alpha \geq \beta\right\}}=\emptyset
$$

para todo $\beta<\kappa$.

Definição 1.2.19 O grau de Lindelöf de $X$ é definido como: $L(X)$ é o menor cardinal infinito $\kappa$ tal que toda cobertura aberta de $X$ tenha alguma subcobertura de cardinalidade $\leq \kappa$.

Definição 1.2.20 [12] O grau de Lindelöf linear de $X$ é definido como: $l l(X)$ é o menor cardinal infinito $\kappa$ tal que toda cobertura aberta de $X$, linearmente ordenada pela inclusão, tenha alguma subcobertura de cardinalidade $\leq \kappa$. 
Definição 1.2.21 A função cardinal "point separating weight" é definida como (apenas para espaços $T_{1}$ ): psw $(X)$ é o menor cardinal infinito $\kappa$ tal que $X$ tenha uma cobertura aberta separante $\mathcal{V}$ com ord $(x, \mathcal{V}) \leq \kappa$ para todo $x \in X$.

Uma cobertura aberta $\mathcal{V}$ de $X$ é separante, se para todo $x \in X$,

$$
\cap\{A: A \in \mathcal{V}, x \in A\}=\{x\} \text {. }
$$

Dada uma cobertura $\mathcal{V}$ e $x \in X$

$$
\operatorname{ord}(x, \mathcal{V})=|\{A: A \in \mathcal{V}, x \in A\}| .
$$

As próximas funções cardinais são definidas primeiro localmente:

Definição 1.2.22 Para $x \in X$, o caráter de $x$ em $X$ é definido como:

$$
\chi(x, X)=\min \{|\mathcal{V}|: \mathcal{V} \text { é uma base de vizinhanças de } x \text { em } X\}+\omega .
$$

Defina então o caráter de $X$ como:

$$
\chi(X)=\sup \{\chi(x, X): x \in X\}
$$

Definição 1.2.23 Para $x \in X$, o tightness de $x$ em $X$ é definido como: $t(x, X)$ é o menor cardinal infinito $\kappa$ tal que, para todo $Y \subset X \operatorname{com} x \in \bar{Y}$, existe $A \in[Y]^{\leq \kappa}$ com $x \in \bar{A}$. Defina então o tightness de $X$ como:

$$
t(X)=\sup \{t(x, X): x \in X\}
$$

Definição 1.2.24 Para $x \in X$, o pseudocaráter de $x$ em $X$ é definido como (apenas para espaços $\left.T_{1}\right): \psi(x, X)$ é o menor cardinal infinito $\kappa$ tal que exista uma família $\mathcal{V}$ de vizinhanças abertas de $x$, com $\cap \mathcal{V}=\{x\}$ e $|\mathcal{V}| \leq \kappa$. Defina então o pseudocaráter de $X$ como:

$$
\psi(X)=\sup \{\psi(x, X): x \in X\}
$$


Definição 1.2.25 [24] Para $x \in X$, o pseudocaráter fechado de $x$ em $X$ é definido como (apenas para espaços $\left.T_{2}\right): \psi_{c}(x, X)$ é o menor cardinal infinito $\kappa$ tal que exista uma família $\mathcal{V}$ de vizinhanças abertas de $x$, com

$$
\cap\{\bar{U}: U \in \mathcal{V}\}=\{x\}
$$

$e|\mathcal{V}| \leq \kappa$. Defina então o pseudocaráter fechado de $X$ como:

$$
\psi_{c}(X)=\sup \left\{\psi_{c}(x, X): x \in X\right\} .
$$

Algumas funções cardinais (como $e, c, s$ e $F$ ) foram definidas por meio de um sup, que pode ser atingido ou não. Se $\phi$ é uma função cardinal, definida como $\phi(X)=\sup \Upsilon(X)$, onde $\Upsilon(X)$ é um conjunto de cardinais infinitos, pode-se definir uma outra função cardinal $\widehat{\phi}$ :

$$
\widehat{\phi}(X)=\min \{\kappa: \forall \mu(\mu \in \Upsilon(X) \Rightarrow \mu<\kappa)\} .
$$

Temos então que $\widehat{\phi}(X)=(\phi(X))^{+}$se $\phi(X) \in \Upsilon(X)$, e $\widehat{\phi}(X)=\phi(X)$ caso contrário.

Definição 1.2.26 Dada uma função cardinal $\phi$, pode-se definir uma função cardinal $h \phi$ :

$$
h \phi(X)=\sup \{\phi(Y): Y \subset X\} .
$$

Uma função cardinal $\phi$ é dita monótona quando $\phi=h \phi$.

Das funções cardinais que definimos acima, $w, s, p s w, \chi, t, \psi$ e $\psi_{c}$ são monótonas.

Definição 1.2.27 [14] Dados um espaço $X$ e $A \subset X$, o caráter de $A$ em $X$ (denotado por $\chi(A, X)$ ) é definido como a menor cardinalidade de uma família $\mathcal{B}(A)$ de abertos em $X$, tal que $A \subset \cap \mathcal{B}(A)$ e dado qualquer aberto $V$ com $A \subset V$, existe $U \in \mathcal{B}(A)$ com $A \subset U \subset V$.

Um espaço Hausdorff $X$ é um espaço de pointwise countable type se para todo $x \in X$ existir um compacto $F \subset X$ tal que $x \in F$ e $\chi(F, X) \leq \aleph_{0}$.

Todo espaço compacto $T_{2}$ (e na verdade todo espaço localmente compacto) é um espaço de pointwise countable type.

Das muitas relações existentes entre estas e outras funções cardinais, enumeramos as mais relevantes para este trabalho. 
Teorema 1.2.28 Seja X um espaço topológico. Temos então que valem as seguintes relações entre funções cardinais em $X$ (desde que essas funções possam ser definidas em $X$ ):

1. $c(X) \leq d(X) \leq w(X)$;

2. $F(X) \leq s(X)=h c(X)=h e(X) \leq w(X)$;

3. $e(X) \leq l l(X) \leq L(X) \leq w(X)$;

4. $\psi(X) \leq p s w(X) \leq w(X)$;

5. $t(X) \leq \chi(X) \leq w(X)$;

6. $\psi(X) \leq \psi_{c}(X) \leq \chi(X)$.

Teorema 1.2.29 Seja $X$ um espaço topológico $T_{2}$. Temos então que:

1. $|X| \leq 2^{L(X) \cdot \chi(X)}$ (Teorema de Arhangel'skiu);

2. $|X| \leq 2^{2^{s(X)}} \quad$ (Desigualdade de Hajnal-Juhász);

3. $\psi_{c}(\bar{S}) \leq 2^{|S|}$ e $|\bar{S}| \leq 2^{|S| \psi_{c}(\bar{S})}$ para qualquer $S \subset X$ (seguem das Proposições 3.1 e 3.2 de [8]).

Teorema 1.2.30 Seja X um espaço topológico regular. Temos então que:

1. $\psi(X)=\psi_{c}(X)$;

2. $\chi(x, D)=\chi(x, \bar{D})$ se $x \in D \subset X$.

Teorema 1.2.31 Seja $X$ um espaço compacto $T_{2}$. Temos então que:

1. $e(X)=l l(X)=L(X)=\omega$;

2. $\psi(X)=\psi_{c}(X)=\chi(X)$ (vale em geral para espaços de pointwise countable type);

3. $p s w(X)=w(X)$;

4. $t(X)=F(X)$. 
Em particular, o teorema acima vale para espaços diádicos. Para estes espaços, vale também:

Teorema 1.2.32 Seja $X$ um compacto diádico. Temos então que:

1. $c(X)=\omega$;

2. $t(X)=\chi(X)=s(X)=w(X)$.

Será para nós mais conveniente trabalhar com a seguinte formulação de $l l(X)$ (equivalente de acordo com a proposição a seguir, que é uma generalização de parte do Teorema 2.1.3 de [15]): o menor cardinal $\kappa$ tal que, toda cobertura aberta $C$ tenha alguma subcobertura $S \operatorname{com} c f(|S|) \leq \kappa$.

Proposição 1.2.33 Sejam X um espaço topológico e $\kappa$ um cardinal infinito. São equivalentes:

(a) toda cobertura aberta $C$, linearmente ordenada por inclusão, tem uma subcobertura $S$ com $|S| \leq \kappa$;

(b) toda cobertura aberta $C$, bem ordenada por inclusão, tem uma subcobertura $S \operatorname{com}|S| \leq \kappa$;

(c) toda cobertura aberta $C$, com $|C|$ regular $>\kappa$, tem uma subcobertura $S$ com $|S|<|C|$

(d) toda cobertura aberta $C$ tem uma subcobertura $S$ com cf $(|S|) \leq \kappa$.

Demonstração. $(a) \Rightarrow(d)$ : Seja $S$ uma subcobertura de $C$, de cardinalidade mínima. Indexando $S$ como $S=\left\{A_{\alpha}: \alpha<|S|\right\}$, defina, para cada $\alpha<|S|$, $B_{\alpha}=\bigcup_{\beta<\alpha} A_{\beta}$. Por (a), a cobertura $U=\left\{B_{\alpha}: \alpha<|S|\right\}$ tem uma subcobertura $U^{\prime}$ com $\left|U^{\prime}\right| \leq \kappa$; pela minimalidade da cardinalidade de $S$, temos então que cf $(|S|) \leq\left|U^{\prime}\right| \leq \kappa$.

$(d) \Rightarrow(c)$ : Imediato.

$(c) \Rightarrow(b)$ : Seja $S$ uma subcobertura de $C$, de tipo de ordem mínimo. Pela minimalidade de $S$ entre as subcoberturas de $C$, ot $(S)$ tem que ser um cardinal regular, e portanto $|S|=$ ot $(S)$. Por (c), $|S| \leq \kappa$, caso contrário $S$ não seria uma subcobertura de $C$ de tipo de ordem mínimo.

$(b) \Rightarrow(a)$ : Basta mostrar que toda cobertura aberta $C$ linearmente ordenada por inclusão, tem alguma subcobertura bem ordenada por inclusão. Para cada ordinal $\alpha$, suponha já definido o conjunto $\left\{A_{\beta}: \beta<\alpha\right\} \subset C$, bem ordenado pela inclusão. Se $X=\bigcup_{\beta<\alpha} A_{\beta}$, já temos o resultado desejado; caso contrário, escolha 
algum $x_{\alpha} \in X \backslash \bigcup_{\beta<\alpha} A_{\beta}$, e algum $A_{\alpha} \in C$ tal que $x_{\alpha} \in A_{\alpha}$. Como $C$ é linearmente ordenado, então $A_{\beta} \subset A_{\alpha}$ para todo $\beta<\alpha$, logo $\left\{A_{\beta}: \beta \leq \alpha\right\}$ é bem ordenado pela inclusão.

Corolário 1.2.34 Se X é um espaço topológico,

$l l(X)=\min \{\kappa:$ toda cobertura aberta $C$ tem subcobertura $S$ com $c f(|S|) \leq \kappa\}$.

O próximo resultado é uma generalização imediata de parte do Teorema 2.1.4 em [15].

Proposição 1.2.35 Sejam $X$ um espaço topológico e $\kappa$ um cardinal infinito. São equivalentes:

(a) $l l(X) \leq \kappa$; em $X$

(b) se $A \subset X$ e $|A|$ é regular $>\kappa$, então $A$ tem ponto de acumulação completo

Demonstração. $(a) \Rightarrow(b)$ : Suponha que exista $A=\left\{x_{\alpha}: \alpha<|A|\right\} \subset X$ contradizendo (b). Para cada $\alpha<|A|$, defina

$$
U_{\alpha}=X \backslash \overline{\left\{x_{\gamma}: \gamma>\alpha\right\}}
$$

Então, $C=\left\{U_{\alpha}: \alpha<|A|\right\}$ é uma cobertura aberta linearmente ordenada por inclusão de $X$. De fato, dado qualquer $x \in X$, como $x$ não é ponto de acumulação completo de $A$ e $|A|$ é regular, existe $\beta<|A|$ tal que $x \in U_{\beta}$. Por (a), existe $\left\{\alpha_{i}: i<\kappa\right\}$ tal que $\left\{U_{\alpha_{i}}: i<\kappa\right\}$ seja subcobertura de $C$, $\log$ c $f(|A|) \leq \kappa$, contradição.

$(b) \Rightarrow(a)$ : Suponha que $l l(X)>\kappa$. Logo pelo item (c) da Proposição 1.2.33, há uma cobertura aberta $U=\left\{U_{\alpha}: \alpha<|U|\right\}$ de $X$ tal que $|U|$ seja um cardinal regular $>\kappa$ e tal que $U$ não tenha subcobertura $U^{\prime}$ com $\left|U^{\prime}\right|<|U|$. Podemos então supor, sem perda de generalidade, que para todo $\alpha<|U|, U_{\alpha} \backslash \bigcup_{\beta<\alpha} U_{\beta} \neq \emptyset$. Defina para cada $\alpha<|U|$,

$$
p_{\alpha} \in U_{\alpha} \backslash \bigcup_{\beta<\alpha} U_{\beta} .
$$

Por (b), $A=\left\{p_{\alpha}: \alpha<|U|\right\}$ tem um ponto de acumulação completo $p \in X$. Mas considerando $\beta<|U|$ tal que $p \in U_{\beta}$, temos que $\left|U_{\beta} \cap A\right| \leq|\beta|<|A|$, contradição. 


\subsection{Submodelos elementares}

Alguns resultados deste trabalho fazem uso de submodelos elementares, que é uma ferramenta que tem se tornado muito importante em Topologia Geral. Nossas principais referências são [10] e a seção sobre submodelos elementares de [9].

Apresentaremos a seguir os conceitos e teoremas sobre submodelos elementares que serão usados neste trabalho. Salvo indicação em contrário, esses conceitos e resultados são provenientes de [9], onde são apresentados e discutidos detalhadamente.

Definição 1.3.1 Seja $M$ um conjunto não vazio. Para cada fórmula $\varphi$, a relativização de $\varphi$ a $M$ é a fórmula $\varphi^{M}$, definida recursivamente por:

- se $\varphi$ é uma fórmula atômica, então $\varphi^{M}$ é a fórmula $\varphi$;

- $(\neg \varphi)^{M}$ é a fórmula $\neg\left(\varphi^{M}\right)$;

- $(\varphi \wedge \phi)^{M}$ é a fórmula $\left(\varphi^{M}\right) \wedge\left(\phi^{M}\right)$;

- $(\exists x(\varphi))^{M}$ é a fórmula $\exists x\left(x \in M \wedge\left(\varphi^{M}\right)\right)$.

Definição 1.3.2 Dada uma fórmula $\varphi\left(x_{1}, \ldots, x_{n}\right)$ e $a_{1}, \ldots, a_{n} \in M$, escrevemos $M \models \varphi\left[a_{1}, \ldots, a_{n}\right]$ se e somente se valer $\varphi^{M}\left(a_{1}, \ldots, a_{n}\right)$.

Definição 1.3.3 Sejam $M, N$ conjuntos com $M \subset N$. Dizemos que $M \prec N$ ( $M$ é um submodelo elementar de $N$ ) se e somente se, para qualquer fórmula $\varphi\left(x_{1}, \ldots, x_{n}\right)$ e quaisquer $a_{1}, \ldots, a_{n} \in M$, tivermos:

$$
M \models \varphi\left[a_{1}, \ldots, a_{n}\right] \text { se e somente se } N \models \varphi\left[a_{1}, \ldots, a_{n}\right] \text {. }
$$

No próximo lema, $\varphi_{x}\left(x_{1}, \ldots, x_{n}\right)$ é a fórmula $\exists x\left(\varphi\left(x_{1}, \ldots, x_{n}, x\right)\right)$.

Lema 1.3.4 (Critério de Tarski) Sejam $M, N$ conjuntos com $M \subset N$. Então $M \prec N$ se e somente se, para qualquer fórmula $\varphi\left(x_{1}, \ldots, x_{n}, x\right)$ e quaisquer $a_{1}, \ldots, a_{n} \in M, N \models \varphi_{x}\left[a_{1}, \ldots, a_{n}\right]$ implicar que exista $a \in M$ tal que $N \models \varphi\left[a_{1}, \ldots, a_{n}, a\right]$. 
Teorema 1.3.5 (Löwenheim-Skolem) Sejam $A$ e $N$ conjuntos com $A \subset N$. Então existe $M \prec N$ tal que $A \subset M$ e $|M| \leq|A|+\omega$.

Deste ponto em diante, quando nos referirmos a um submodelo elementar $M$, este será submodelo elementar de um $H_{\theta}\left(H_{\theta}=\{A:|\operatorname{trcl}(A)|<\theta\}\right.$, ver [34] e [9]), $\operatorname{com} \theta$ cardinal não enumerável, regular e "suficientemente grande" para que $H_{\theta}$ possa ser tomado como universo para os objetos sendo estudados.

Lema 1.3.6 Sejam $\kappa$ um cardinal e $M \prec H_{\theta}$ tais que $\kappa \in M$ e $\kappa \subset M$. Então,

$$
\forall A \in M(|A| \leq \kappa \Rightarrow A \subset M)
$$

Lema 1.3.7 Sejam $\kappa$ um cardinal infinito $e M \prec H_{\theta}$ com $\kappa \cup\{\kappa\} \subset M$. Então $\eta=\kappa^{+} \cap M$ é um ordinal limite.

Definição 1.3.8 Sejam $\kappa$ um cardinal infinito e $M \prec H_{\theta}$. Dizemos que $M$ é $\kappa$-covering se para todo $A \in[M]^{\leq \kappa}$ existe $B \in M$ tal que $A \subset B$ e $|B| \leq \kappa$.

O lema a seguir é uma imediata generalização para cardinais quaisquer do Lema $3.3 \mathrm{em}[31]$.

Lema 1.3.9 Se $M$ é um submodelo elementar $\kappa$-covering, com $\kappa \cup\{\kappa\} \subset M$, então $\kappa^{+} \subset M$.

Demonstração. Definindo $\alpha=M \cap \kappa^{+}$, basta mostrar que $\alpha=\kappa^{+}$. De acordo com o Lema 1.3.7, $\alpha$ é um ordinal. Suponha que $\alpha<\kappa^{+}$. Como $|\alpha| \leq \kappa$ e $M$ é $\kappa$-covering, existe $A \in M$ tal que $\alpha \subset A$ e $|A| \leq \kappa$. Definindo $B=A \cap \kappa^{+}$, temos que $B \in M, \alpha \subset B$ e $|B| \leq \kappa$. Temos então pelo Lema 1.3.6 que $B \subset M$, logo $B \subset M \cap \kappa^{+}=\alpha$. Portanto $\alpha=B \in M$, contradição, pois $\alpha \notin \alpha$.

Lema 1.3.10 Sejam $\kappa$ um cardinal infinito e $A \subset H_{\theta}$ com $|A| \leq \kappa^{+}$. Então existe $M \prec H_{\theta} \kappa$-covering com $A \subset M e|M| \leq \kappa^{+}$.

Demonstração. Se tivermos $|A| \leq \kappa$, temos imediatamente o resultado pelo Lema 1.1.12 de [9]. Se $|A|=\kappa^{+}$, aplique o Lema 1.1.12 de [9] $\operatorname{com} A^{\prime}=\kappa \cup\left\{\kappa, \kappa^{+}, A\right\}$, pelo Lema 1.3.9 temos $\kappa^{+} \subset M$, logo pelo Lema 1.3.6, $A \subset M$. 
Definição 1.3.11 [30][31] Dado um espaço topológico $(X, \tau), X_{M}$ denota o seguinte espaço topológico: o conjunto $X \cap M$, munido da topologia gerada por

$$
\{U \cap M: U \in \tau \cap M\} .
$$

Note que, de acordo com definição acima, a topologia de $X_{M}$ ou coincide ou será menos fina que a topologia de subespaço de $X \cap M$.

\subsection{Reflexão de funções cardinais}

Nesta seção iremos estabelecer os conceitos relacionados à reflexão de funções cardinais, bem como algumas relações e propriedades. Nossa principal referência será [22].

Definição 1.4.1 Sejam $\phi$ uma função cardinal, $\kappa$ um cardinal infinito e $S$ uma classe de espaços topológicos. Então a expressão " $\phi$ reflete $\kappa$ para $S$ " significa:

se $X \in S$ e $\phi(X) \geq \kappa$, então existe $Y \subset X$ tal que $|Y| \leq \kappa$ e $\phi(Y) \geq \kappa$.

Se $S$ for a classe de todos os espaços topológicos, podemos dizer simplesmente " $\phi$ reflete $\kappa "$.

Definição 1.4.2 Sejam $\phi$ uma função cardinal, $\kappa$ um cardinal infinito e $S$ uma classe de espaços topológicos. Então a expressão " $\phi$ reflete fortemente $\kappa$ para $S$ " significa:

se $X \in S$ e $\phi(X) \geq \kappa$, então $\exists Y \in[X]^{\leq \kappa}$ com $\phi(Z) \geq \kappa$ sempre que $Y \subset Z \subset X$.

Definição 1.4.3 Sejam $\phi$ uma função cardinal, $\kappa$ um cardinal infinito e $S$ uma classe de espaços topológicos. Então a expressão " $\phi$ satisfaz $I U(\kappa)$ para $S^{\prime \prime 2}$ significa: se $X \in S, \phi(X) \geq \kappa$ e $X=\cup\left\{X_{\alpha}: \alpha<\lambda\right\}$, com $\kappa<\lambda$, $\lambda$ regular e $\left\{X_{\alpha}: \alpha<\lambda\right\}$ crescente, então existe $\alpha<\lambda$ tal que $\phi\left(X_{\alpha}\right) \geq \kappa$.

\footnotetext{
${ }^{2} I U=$ "increasing union"
} 
Definição 1.4.4 Sejam $\phi$ uma função cardinal, $\kappa$ um cardinal infinito e $S$ uma classe de espaços topológicos. Então a expressão " $\phi$ tem a propriedade de Darboux em $\kappa$ para $S$ " significa:

$$
\text { se } X \in S \text { e } \phi(X)>\kappa \text {, então existe } Y \subset X \text { tal que } \phi(Y)=\kappa \text {. }
$$

Teorema 1.4.5 Dada uma função cardinal $\phi$, um cardinal infinito $\kappa$ e uma classe de espaços topológicos $S$, temos que:

i) se $\phi$ reflete fortemente $\kappa$ para $S$, então $\phi$ reflete $\kappa$ para $S$;

ii) se $\phi$ é monótona e reflete $\kappa$ para $S$, então $\phi$ reflete fortemente $\kappa$ para $S$;

iii) se $\phi$ reflete fortemente $\kappa$ para $S$, então $\phi$ satisfaz $I U(\kappa)$ para $S$;

iv) suponha que $\phi$ satisfaça: $\phi(X) \leq|X|+\omega$ para todo espaço $X$. Assim, se $\phi$ reflete $\kappa$ para $S$, então $\phi$ tem a propriedade de Darboux em $\kappa$ para $S$.

Lema 1.4.6 Se $\phi$ reflete fortemente todos os cardinais sucessores para $S$, então $\phi$ reflete fortemente todos os cardinais infinitos para $S$.

Neste trabalho, quando dissermos " $\phi$ reflete $\kappa$ para $X$ ", isto deve ser entendido como " $\phi$ reflete $\kappa$ para $\{X\} "$.

Um dos primeiros resultados de reflexão, em 1980, foi o Teorema de HajnalJuhász de reflexão do peso [20]:

Teorema 1.4.7 $w$ reflete todos os cardinais infinitos. 


\section{Capítulo 2}

\section{Reflexões em funções locais}

\subsection{Introdução}

Neste capítulo estudaremos propriedades de reflexão para funções cardinais locais, como $\psi$ e $\chi$, além da função cardinal psw. Estas três funções cardinais são monótonas, portanto, de acordo com o Teorema 1.4.5 e o Lema 1.4.6, se $\phi \in\{\chi, \psi, p s w\}$ e $S$ for uma classe de espaços topológicos, para que $\phi$ reflita todos os cardinais infinitos para $S$, basta que $\phi$ reflita todos os cardinais sucessores para $S$.

Estes são os principais resultados de reflexão descritos na literatura para $\psi$ e psw [22]:

Teorema 2.1.1 $\left(2^{\kappa}=\kappa^{+}\right)$Se $\phi \in\{\psi, p s w\}$, então $\phi$ reflete $\kappa^{+}$para a classe dos espaços compactos $T_{2}$.

Corolário 2.1.2 (GCH) Se $\phi \in\{\psi, p s w\}$, então $\phi$ reflete todos os cardinais infinitos para a classe dos espaços compactos $T_{2}$.

Relacionado aos resultados acima, este é um dos principais problemas em aberto sobre reflexão de funções cardinais locais [22][26]:

Problema 2.1.3 Seja $\phi \in\{\psi, p s w\}$. Pode-se mostrar em ZFC que $\phi$ reflete $\kappa^{+}$ para a classe dos espaços compactos $T_{2}$ ? Ou em particular, que $\phi$ reflete $\omega_{1}$ para a classe dos espaços compactos $T_{2}$ ?

Na literatura, o único resultado positivo que encontramos sobre reflexão de $\psi$ ou psw, em ZFC, para espaços compactos $T_{2}$ é: 
Teorema 2.1.4 [7] Se $\phi \in\{\psi, p s w\}$, então $\phi$ reflete $\kappa^{+}$para a classe dos espaços diádicos.

Nas próximas seções deste capítulo, com exceção da última, nós buscaremos respostas parciais para o Problema 2.1.3. Na última seção, usaremos submodelos elementares para obter alguns resultados de reflexão para $\chi$ e $\psi$, incluindo uma relação entre reflexão e propriedade de Darboux para $\chi$.

\subsection{Espaços diádicos}

A demonstração em [7] do Teorema 2.1.4, apesar de aparentemente curta, faz uso do Teorema de Efimov-Gerlits-Hagler ([13]), o que torna a demonstração como um todo não elementar e muito complexa. Apresentamos a seguir uma demonstração mais simples do mesmo resultado. Primeiro, apresentamos um lema, cujo enunciado é semelhante ao Problema 3.12.12 (i) de [14], mas adaptado às nossas necessidades.

Lema 2.2.1 Se $X$ é um espaço diádico e $\chi\left(x_{0}, X\right) \geq \lambda \geq \omega_{1}$, então existe $M \in$ $[X]^{\lambda}$ tal que $M$ é discreto e $M \cup\left\{x_{0}\right\}$ é a compactificação de Alexandroff de $M$.

Demonstração. Como $X$ é diádico, existe uma função

$$
f: C=\prod_{s \in S} D_{s} \rightarrow X
$$

contínua e sobrejetora (com $|S| \geq \omega$, e para todo $s \in S, D_{s}$ é o espaço discreto $\{0,1\})$.

Dados $u, v \in C$, denote por $\Delta(u, v)$ o conjunto $\left\{s \in S: \pi_{s}(u) \neq \pi_{s}(v)\right\}$.

Defina $A=f^{-1}\left(x_{0}\right)$ e

$$
S_{0}=\{s \in S: \exists a \in A(\exists b \in C \backslash A(\Delta(a, b)=\{s\}))\} .
$$

Fixe agora funções $a, b: S_{0} \rightarrow C$ tais que, para todo $s \in S_{0}, a(s) \in A, b(s) \in C \backslash A$ e $\Delta(a(s), b(s))=\{s\}$.

Vejamos agora que

$$
A=\pi_{S_{0}}(A) \times \prod_{s \in S \backslash S_{0}} D_{s}
$$

É evidente que

$$
A \subset \pi_{S_{0}}(A) \times \prod_{s \in S \backslash S_{0}} D_{s}
$$


basta então mostrar a inclusão oposta. Seja $u \in \pi_{S_{0}}(A) \times \prod_{s \in S \backslash S_{0}} D_{s}$ e suponha que $u \notin A$. Seja $v \in A$ tal que $\pi_{S_{0}}(v)=\pi_{S_{0}}(u)$. Como $A$ é fechado, existe $T \subset S$ finito tal que

$$
\left(\pi_{T}(u) \times \prod_{s \in S \backslash T} D_{s}\right) \subset C \backslash A .
$$

Considerando agora o elemento $w \in C$ tal que $\pi_{T}(w)=\pi_{T}(u)$ e $\pi_{S \backslash T}(w)=$ $\pi_{S \backslash T}(v)$, é fácil ver que $w \notin A$ e que $\Delta(w, v) \subset T \backslash S_{0}$. Se $\Delta(w, v)=\emptyset, w=v$, contradição. Caso contrário, podemos expressar $\Delta(w, v)$ como $\left\{t_{1}, t_{2}, \ldots, t_{k}\right\}$ e proceder da seguinte forma. Defina $w_{0}=w$, e para cada $n \in\{1,2, \ldots, k\}, w_{n} \in C$ como sendo o elemento tal que $\pi_{t_{n}}\left(w_{n}\right)=\pi_{t_{n}}(v)$ e $\pi_{S \backslash\left\{t_{n}\right\}}\left(w_{n}\right)=\pi_{S \backslash\left\{t_{n}\right\}}\left(w_{n-1}\right)$; temos que $w_{n-1} \notin A$ implica $w_{n} \notin A$, pois $t_{n} \notin S_{0}$. Mas $w_{k}=v$, contradição. Mostramos assim que

$$
A=\pi_{S_{0}}(A) \times \prod_{s \in S \backslash S_{0}} D_{s} .
$$

Agora vamos mostrar que $\left|S_{0}\right| \geq \lambda$. Por absurdo, seja $J_{0}$ o conjunto de todos os subconjuntos finitos de $S_{0}\left(\operatorname{logo},\left|J_{0}\right|<\lambda\right)$. Para cada $a \in A$ e $j \in J_{0}$, defina

$$
U_{a, j}=\pi_{j}(a) \times \prod_{s \in S \backslash j} D_{s} .
$$

Então, para cada $j \in J_{0}, U_{j}=\bigcup_{a \in A} U_{a, j}$ é um aberto tal que $A \subset U_{j}$. Defina então, para cada $j \in J_{0}, V_{j}=X \backslash f\left[C \backslash U_{j}\right]$. É fácil ver que $V_{j}$ é uma vizinhança aberta de $x_{0}$. Basta agora mostrar que $\bigcap_{j \in J_{0}} V_{j}=\left\{x_{0}\right\}$, o que levará a uma contradição com $\psi\left(x_{0}, X\right)=\chi\left(x_{0}, X\right) \geq \lambda$. Suponha que existe $z \in \bigcap_{j \in J_{0}} V_{j}$, com $z \neq x_{0}$. Escolha algum $y \in f^{-1}(z)$. É fácil ver que $y \in \bigcap_{j \in J_{0}} U_{j}$ e $y \notin A$. Seja então $R \subset S$, finito, tal que

$$
\left(\pi_{R}(y) \times \prod_{s \in S \backslash R} D_{s}\right) \subset C \backslash A .
$$

Como $y \in \bigcap_{j \in J_{0}} U_{j}$, em particular temos que $y \in U_{R \cap S_{0}}$, logo existe $a_{y} \in A$ tal que $y \in U_{a_{y}, R \cap S_{0}}$, o que significa que

$$
\pi_{R \cap S_{0}}(y)=\pi_{R \cap S_{0}}\left(a_{y}\right) .
$$

Considere então o elemento $b_{y} \in C$ tal que $\pi_{R}\left(b_{y}\right)=\pi_{R}(y)$ e $\pi_{S \backslash R}\left(b_{y}\right)=\pi_{S \backslash R}\left(a_{y}\right)$. Por um lado, como

$$
b_{y} \in \pi_{R}(y) \times \prod_{s \in S \backslash R} D_{s},
$$

$b_{y} \notin A$. Por outro lado, como $\pi_{S \backslash R}\left(b_{y}\right)=\pi_{S \backslash R}\left(a_{y}\right)$ e

$$
\pi_{R \cap S_{0}}\left(b_{y}\right)=\pi_{R \cap S_{0}}(y)=\pi_{R \cap S_{0}}\left(a_{y}\right),
$$


então $\Delta\left(b_{y}, a_{y}\right) \subset R \backslash S_{0}$, o que implica $\pi_{S_{0}}\left(b_{y}\right)=\pi_{S_{0}}\left(a_{y}\right)$, logo

$$
b_{y} \in \pi_{S_{0}}(A) \times \prod_{s \in S \backslash S_{0}} D_{s}=A .
$$

Contradição, o que mostra que $\bigcap_{j \in J_{0}} V_{j}=\left\{x_{0}\right\}$. Mostramos assim que $\left|S_{0}\right| \geq \lambda$.

Vejamos agora que $B=b\left[S_{0}\right]$ (a imagem da função $b$ ) é tal que $|B| \geq \lambda$. Para isto basta mostrar que, dado qualquer $\widetilde{s} \in S_{0}, b^{-1}(b(\widetilde{s}))$ é finito. Como $b(\widetilde{s}) \notin A$, existe $T \subset S$, finito, tal que

$$
\left(\pi_{T}(b(\widetilde{s})) \times \prod_{s \in S \backslash T} D_{s}\right) \subset C \backslash A .
$$

Deste modo, $b^{-1}(b(\widetilde{s}))$ é finito pois $b^{-1}(b(\widetilde{s})) \subset T$ - se houvesse $s \in b^{-1}(b(\widetilde{s})) \backslash T$, teríamos

$$
a(s) \in \pi_{T}(b(\widetilde{s})) \times \prod_{s \in S \backslash T} D_{s},
$$

contradição.

Vamos agora mostrar que todos os pontos de acumulação de $B$ estão em $A$. Faremos isso mostrando que, dado qualquer $u \in C \backslash A, u$ não é ponto de acumulação de $B$. Como $A$ é fechado, existe $T \subset S$ finito tal que

$$
U=\left\{c \in C: \pi_{T}(c)=\pi_{T}(u)\right\}
$$

é uma vizinhança aberta de $u$, com $U \cap A=\emptyset$. Se $s \in S_{0} \backslash T$, então $b(s) \notin U$ (pois neste caso $\left.\pi_{T}(a(s))=\pi_{T}(b(s))\right)$. Assim,

$$
U \cap\left(C \backslash\left(b\left[S_{0} \cap T\right] \backslash\{u\}\right)\right)
$$

é uma vizinhança aberta de $u$ que não intercepta nenhum ponto de $B$, com possível exceção do próprio $u$.

Defina agora $\widetilde{M}=f[B]$. Queremos mostrar que $|\widetilde{M}| \geq \lambda$. Para isto basta mostrar que, dado qualquer $b \in B$,

$$
B_{b}=f^{-1}(f(b)) \cap B
$$

é finito. Se $B_{b}$ fosse infinito, teria um ponto de acumulação $a_{b} \in A$, mas

$$
C \backslash f^{-1}(f(b))
$$

é uma vizinhança aberta de $a_{b}$ que não intercepta $B_{b}$. 
Vejamos agora que o conjunto dos pontos de acumulação de $\widetilde{M}$ é $\left\{x_{0}\right\}$. Para isto basta mostrar que, se $z \in X \backslash\left\{x_{0}\right\}$, então $z$ não é ponto de acumulação de $\widetilde{M}$ (então $x_{0}$ será ponto de acumulação de $\widetilde{M}$ pois $\widetilde{M}$ é infinito e $X$ é compacto). Como $f^{-1}(z) \cap A=\emptyset$, nenhum $y \in f^{-1}(z)$ é ponto de acumulação de $B$. Então, para cada $y \in f^{-1}(z)$, seja $U_{y}$ uma vizinhança aberta de $y$ tal que $U_{y} \cap B \subset\{y\}$. Logo $U=\bigcup_{y \in f^{-1}(z)} U_{y}$ é um aberto tal que $f^{-1}(z) \subset U$ e, se $b \in B \cap U$, então $f(b)=z$. Defina $V=X \backslash f[C \backslash U]$. É fácil ver que $V$ é uma vizinhança aberta de $z$. Temos ainda que $V \cap \widetilde{M} \subset\{z\}$ (se existisse $m \in \widetilde{M} \cap(V \backslash\{z\})$, escolha algum $b \in f^{-1}(m) \cap B$, pela definição de $V$ teríamos $b \in U$, contradição).

Como $\left\{x_{0}\right\}$ é o conjunto dos pontos de acumulação de $\widetilde{M}, \widetilde{M}$ é discreto, e $\widetilde{M} \cup\left\{x_{0}\right\}$ é compacto. Finalmente, escolha qualquer $M \in[\widetilde{M}]^{\lambda}$. Temos que $M$ é discreto e $M \cup\left\{x_{0}\right\}$ é a compactificação de Alexandroff de $M$.

Teorema 2.2.2 Sejam $\phi \in\{\psi, p s w\}$ e $\kappa$ um cardinal infinito. Então $\phi$ reflete $\kappa^{+}$ para a classe

$$
\left\{X: X \text { é compacto } T_{2} \text { e existe } Y \subset X \text { diádico, } \operatorname{com} \phi(Y) \geq \kappa^{+}\right\} \text {. }
$$

Demonstração. Seja $Y \subset X$ um diádico $\operatorname{com} \psi(Y) \geq \kappa^{+}$. Então existe $x_{0} \in Y$ tal que $\chi\left(x_{0}, Y\right)=\psi\left(x_{0}, Y\right) \geq \kappa^{+}$. Pelo Lema 2.2.1, existe $M \in[Y]^{\kappa^{+}} \subset[X]^{\kappa^{+}}$ discreto, com $N=M \cup\left\{x_{0}\right\}$ a sua compactificação de Alexandroff. Então é fácil ver que $\psi(N)=\psi\left(x_{0}, N\right)=\kappa^{+}$.

Seja $Y \subset X$ um diádico com $p s w(Y) \geq \kappa^{+}$. Como $Y$ é diádico, $\psi(Y)=$ $w(Y)=p s w(Y)$. De acordo com o parágrafo anterior, existe então $N \in[Y]^{\kappa^{+}}$, compacto, com $\psi(N)=\kappa^{+}$, o que mostra que psw $(N) \geq \kappa^{+}$.

A estratégia da demonstração desse resultado, foi obter um subespaço que seja exatamente a compactificação de Alexandroff de um discreto de cardinalidade $\kappa^{+}$. Como todo diádico é um compacto c.c.c. (ou seja, tem celularidade enumerável), seria natural tentar estender essa estratégia, de diádicos para compactos c.c.c.

Entretanto, isso não pode funcionar, pois, por exemplo, $\beta \omega$ não possui subespaços compactos de cardinalidade $\omega_{1}$. Entretanto, no caso particular de reflexão de $\omega_{1}$, podemos estender o teorema anterior:

Proposição 2.2.3 Seja $\phi \in\{\psi, p s w\}$. Então $\phi$ reflete $\omega_{1}$ para a classe dos espaços $X$, compactos $T_{2}$, que satisfaçam pelo menos uma das condições:

1. existe $Y \subset X$ diádico, $\operatorname{com} \phi(Y) \geq \omega_{1}$; 
2. existe um subespaço de $X$ homeomorfo a $\beta \omega$.

Demonstração. Basta mostrar (2), pois se tivermos (1), o resultado segue do Teorema 2.2.2.

Pelo Corolário 4.4.5 de [45], existe um subespaço $S$ de $\omega^{*}$ (e portanto de $\beta \omega$ ) de cardinalidade $\omega_{1}$, onde existe um ponto de $S$ tal que as vizinhanças abertas são as co-enumeráveis, e todos os demais pontos de $S$ são isolados. Claramente, $\operatorname{psw}(S) \geq \psi(S)=\omega_{1}$.

\subsection{Espectros de caráter e convergência}

Nesta seção faremos uso de conceitos definidos e desenvolvidos em [29]: espectro de caráter e espectro de convergência. Nesse artigo, Juhász e Weiss utilizam esses conceitos para reformular certos problemas topológicos em aberto e apresentar algumas soluções. Com relação à reflexão de funções cardinais, nesse artigo há uma aplicação à reflexão do caráter, mas nesta seção aplicaremos esses conceitos no estudo da reflexão de $\psi$ e $p s w$.

Vejamos primeiro as seguintes definições e resultados:

Definição 2.3.1 [29] Dizemos que uma sequência $\left(x_{\alpha}: \alpha<\kappa\right)$ converge para um ponto $x$ em um espaço topológico $X$ (o que denotamos por $x_{\alpha} \rightarrow x$ ), se para toda vizinhança aberta $U$ de $x$ existe algum $\beta<\kappa$ tal que $x_{\alpha} \in U$ sempre que $\beta \leq \alpha<\kappa$.

Definição 2.3.2 [29] Dizemos que um subconjunto infinito $A$ de um espaço topológico $X$ converge para um ponto $x$ (o que denotamos por $A \rightarrow x$ ), se para toda vizinhança aberta $U$ de $x$ tivermos $|A \backslash U|<|A|$.

Proposição 2.3.3 [29] Se uma sequência $\left(x_{\alpha}: \alpha<\kappa\right)$ converge para um ponto $x$, então o conjunto $A=\left\{x_{\alpha}: \alpha<\kappa\right\}$ também converge para $x$. Por outro lado, se $A \rightarrow x$ e $|A|$ for regular, então se $\left\{x_{\alpha}: \alpha<|A|\right\}$ for qualquer enumeração injetora de $A, x_{\alpha} \rightarrow x$ (a regularidade de $|A|$ é essencial aqui).

Proposição 2.3.4 [29] Em um compactum, $A \rightarrow x$ se e somente se $x$ for o único ponto de acumulação completo de A.

Podemos agora definir os seguintes espectros de cardinais: 
Definição 2.3.5 [29] Se p for um ponto não isolado de $X$, definimos o espectro de caráter de $p$ em $X$ como

$$
\chi S(p, X)=\{\chi(p, Y): p \text { é não isolado em } Y \subset X\} .
$$

Definimos então o espectro de caráter de X como

$$
\chi S(X)=\bigcup\{\chi S(x, X): x \text { é não isolado em } X\} .
$$

Definição 2.3.6 [29] Dado $p \in X$, definimos o espectro de convergência de p em $X$ como

$$
c S(p, X)=\{|A|: A \subset X \text { e } A \rightarrow p\} .
$$

Definimos então o espectro de convergência de $X$ como

$$
c S(X)=\bigcup\{c S(x, X): x \in X\} .
$$

Definição 2.3.7 [29] Dado $p \in X$, definimos o espectro de convergência discreto de $p$ em $X$ como

$$
d c S(p, X)=\{|D|: D \subset X \text { é discreto e } D \rightarrow p\} .
$$

Definimos então o espectro de convergência discreto de $X$ como

$$
d c S(X)=\bigcup\{d c S(x, X): x \in X\}
$$

É evidente que $d c S(X) \subset c S(X)$. A demonstração do próximo resultado é baseada em argumentos desenvolvidos no texto de [29].

Proposição 2.3.8 Se $X$ é um espaço compacto $T_{2}$, e $\kappa \in \chi S(X)$, então

$$
\{\kappa, c f(\kappa)\} \subset c S(X) .
$$

Demonstração. Se $\kappa \in \chi S(X)$, então existe algum $p \in X$, não isolado, tal que $\kappa \in \chi S(p, X)$. Logo existe algum $Y \subset X$, com $p \in Y$, tal que $p$ é não isolado em $Y$ e $\chi(p, Y)=\kappa$. Como $X$ é compacto $T_{2}$ (e portanto regular),

$$
\psi(p, \bar{Y})=\chi(p, \bar{Y})=\chi(p, Y)
$$


então pode-se facilmente mostrar que existe uma sequência injetora $\left(x_{\alpha}: \alpha<\kappa\right)$ convergindo para $p$. Se $I=\left\{\alpha_{\beta}: \beta<c f(\kappa)\right\}$ for crescente e cofinal em $\kappa$, então temos também que $x_{\alpha_{\beta}} \rightarrow p$. Definindo agora $A=\left\{x_{\alpha}: \alpha<\kappa\right\}$ e $B=$ $\left\{x_{\alpha_{\beta}}: \beta<c f(\kappa)\right\}$, temos que $A \rightarrow p$ e $B \rightarrow p$, logo

$$
\{|A|,|B|\}=\{\kappa, c f(\kappa)\} \subset c S(p, X) \subset c S(X) .
$$

Teorema 2.3.9 Sejam $\kappa$ um cardinal infinito regular e $\phi \in\{\psi, p s w\}$. Temos então que $\phi$ reflete $\kappa$ para a classe dos espaços $\{X: \kappa \in c S(X)\}$.

Demonstração. Se $\kappa \in c S(X)$, então existe algum $p \in X$ tal que $\kappa \in c S(p, X)$. Portanto existe $A \in[X]^{\kappa}$ tal que $A \rightarrow p$. Podemos supor sem perda de generalidade que $p \in A$. Temos então que $\psi(p, A)=\kappa$, pois se tivéssemos $\psi(p, A)=$ $\lambda<\kappa$, existiria uma família $\left\{U_{\alpha}: \alpha<\lambda\right\}$ de vizinhanças abertas de $p$ em $A$ tal que $\bigcap_{\alpha<\lambda} U_{\alpha}=\{p\}$. Mas então teríamos $A \backslash\{p\}=\bigcup_{\alpha<\lambda} A \backslash U_{\alpha}$, o que contradiria a regularidade de $\kappa$.

Deste modo, psw $(A)=\psi(A)=\kappa$, logo psw e $\psi$ refletem $\kappa$ para $X$.

Faremos uso dos seguintes resultados:

Proposição 2.3.10 [29] Seja $\kappa$ um cardinal infinito. Se $X$ é um compactum com $\chi(X)>2^{\kappa}$, então $\kappa^{+} \in d c S(X)$.

Proposição 2.3.11 [29] Se @ é um cardinal infinito e $X$ é um compactum com $\widehat{F}(X)>\varrho=c f(\varrho)>\omega$ ent $\tilde{o} o \varrho \in \chi S(X)$.

Proposição 2.3.12 [29] Suponha que $X$ é um espaço regular e $\varrho, \mu$ são cardinais infinitos tais que $\widehat{F}(X) \leq \varrho \leq c f(\mu)$, e ainda que $p \in X$ é tal que $\psi(p, X) \geq \mu$. Então:

1. existe um discreto $D \in[X]^{<\varrho}$ com $p \in \bar{D}$ e $\psi(p, \bar{D}) \geq \mu$, ou

2. existe um discreto $D \in[X]^{\varrho}$ tal que $D \rightarrow p$.

Podemos agora encontrar mais condições suficientes para a reflexão de $\psi$ e $p s w$.

Teorema 2.3.13 Sejam $\kappa$ um cardinal infinito e $X$ um espaço compacto $T_{2}$. Para que $\psi$ reflita $\kappa^{+}$para $X$, é suficiente que ocorra uma das condições: 
1. $\exists p \in X\left(\psi(p, X)=\kappa^{+}\right)$;

2. $\psi(X) \leq \kappa^{+}$;

3. $\psi(X)>2^{\kappa}$;

4. $t(X) \geq \kappa^{+}$

5. $\forall S \in[X]^{\leq \kappa}\left(\psi(\bar{S}) \leq \kappa^{+}\right)$.

Demonstração. (1): temos que

$$
\kappa^{+}=\chi(p, X) \in \chi S(p, X) \subset c S(X),
$$

e basta aplicar o Teorema 2.3.9;

(2): se não tivermos (1), então $\psi(X) \leq \kappa$, e o resultado é imediato;

(3): pela Proposição 2.3.10, $\kappa^{+} \in d c S(X) \subset c S(X)$;

(4): $F(X)=t(X) \geq \kappa^{+}$implica que $\widehat{F}(X)>\kappa^{+}$, logo pela Proposição 2.3.11 $\kappa^{+} \in \chi S(X) \subset c S(X)$.

(5): suponha que não temos (2) nem (4). Neste caso, $\psi(X) \geq \kappa^{++}$, logo $\psi(p, X) \geq \kappa^{++}$para algum $p \in X$. Além disso, $F(X)=t(X) \leq \kappa, \operatorname{logo} \widehat{F}(X) \leq$ $\kappa^{+}$. Podemos então aplicar a Proposição 2.3.12, com $\varrho=\kappa^{+}$e $\mu=\kappa^{++}$. Por (5), não pode ocorrer a conclusão (1) da Proposição 2.3.12, então temos (2), logo

$$
|D|=\varrho=\kappa^{+} \in c S(p, X) \subset c S(X) .
$$

Com base no item (5) do teorema acima, se existir um espaço $X$ que seja contra-exemplo consistente do enunciado " $\psi$ reflete $\kappa^{+}$para a classe dos espaços compactos $T_{2}$ ", haverá $Y \subset X$, compacto, com $c(Y) \leq d(Y) \leq \kappa$, que também será contra-exemplo consistente do mesmo enunciado. Portanto, o problema do pseudocaráter refletir ou não $\omega_{1}$ é equivalente na classe dos espaços compactos $T_{2}$ ou na classe dos espaços compactos $T_{2}$ c.c.c.

Corolário 2.3.14 Sejam $\kappa$ um cardinal infinito e $X$ um espaço compacto $T_{2}$. Para que psw reflita $\kappa^{+}$para $X$, é suficiente que ocorra uma das condições:

1. $\operatorname{psw}(X) \leq \kappa$;

2. $\psi(X)=\kappa^{+}$ou $\psi(X)>2^{\kappa}$; 
3. $t(X) \geq \kappa^{+}$;

4. $\psi(X) \geq \kappa^{+} e \forall S \in[X]^{\leq \kappa}\left(\psi(\bar{S}) \leq \kappa^{+}\right)$.

Demonstração. Se $X$ é um espaço compacto $T_{2} \operatorname{com} \psi(X) \geq \kappa^{+}$, " $\psi$ reflete $\kappa^{+}$ para $X$ " implica "psw reflete $\kappa^{+}$para $X^{\text {". }}$

Seja $\phi \in\{\psi, p s w\}$. Com base no Teorema 2.3.13 e no Corolário 2.3.14, pode-se deduzir de outra forma o Teorema 2.2.2, pois se $X$ é compacto diádico, $t(X)=$ $\psi(X)=p s w(X)$. E também a Proposição 2.2.3, pois $t(\beta \omega)=2^{\omega}$ (Exemplo 7.22 em $[21])$.

\subsection{Classificação dos possíveis contra-exemplos}

Nesta seção, iremos apresentar algumas caracterizações adicionais dos possíveis contra-exemplos consistentes do enunciado " $\phi$ reflete $\kappa^{+}$para a classe dos espaços compactos $T_{2}$ ", com $\phi \in\{\psi, p s w\}$. Vejamos as seguintes definições, que são relativas a um problema também abordado em [29]:

Definição 2.4.1 [18] Seja $X$ um compactum. Dizemos que $x \in X$ é um $\boldsymbol{K}$ ponto se para nenhum cardinal regular não-enumerável @ existir uma @-sequencia em $X \backslash\{x\}$ convergindo para $x$.

Definição 2.4.2 [18] Seja $X$ um compactum. Dizemos que $x \in X$ é um $\boldsymbol{A B}$ ponto se cf $(\chi(x, Y)) \leq \omega$ para todo $Y \subset X$ com $x \in Y$.

Definição 2.4.3 [18] Um compactum $X$ é um $\boldsymbol{K}$-compactum (AB-compactum) se todos os seus pontos forem K-pontos (AB-pontos).

É fácil ver que um compactum é um K-compactum se e somente se no seu espectro de convergência não houver nenhum cardinal regular não-enumerável; e é um AB-compactum se e somente se no seu espectro de caráter houver apenas cardinais de cofinalidade enumerável. Pode-se ver facilmente pela Proposição 2.3.8 que todo K-compactum é um AB-compactum.

Precisaremos do seguinte conceito (ver [3]): 
Definição 2.4.4 Dados um espaço $X$ e $x \in X$, definimos Hus $(x, X)$ como sendo $o$ menor cardinal infinito $\tau$ satisfazendo a seguinte condição: para todo $A \subset$ $X \backslash\{x\}$ tal que $|A|$ seja um cardinal regular $\geq \tau$, existe uma vizinhança aberta $U$ de $x$ tal que $|A \backslash U|=|A|$. Definimos então Hus $(X)$ como $\sup \{H u s(x, X): x \in X\}$.

Não é difícil ver que um compactum $X$ é um K-compactum se e somente se $\operatorname{Hus}(X) \leq \omega_{1}$.

O próximo resultado generaliza a Proposição 4 de [3].

Proposição 2.4.5 Sejam $X$ um espaço compacto $T_{2}, x \in X$ e $\kappa$ um cardinal infinito. Então Hus $(x, X) \leq \kappa^{+}$se e somente se $l l(X \backslash\{x\}) \leq \kappa$.

Demonstração. Considerando a definição de quando um subconjunto converge para um ponto, temos que $\operatorname{Hus}(x, X) \leq \kappa^{+}$se e somente se, para todo $A \subset X \backslash\{x\}$, $\operatorname{com}|A|$ regular $\geq \kappa^{+}, A$ não converge para $x$.

Como $A \rightarrow x$ se e somente se $x$ for o único ponto de acumulação completo de $A$, temos que Hus $(x, X) \leq \kappa^{+}$se e somente se, para todo $A \subset X \backslash\{x\}$, com $|A|$ regular $\geq \kappa^{+}, A$ tem outro ponto de acumulação completo, ou seja, tem ponto de acumulação completo em $X \backslash\{x\}$. Essa última condição, por sua vez, equivale a $l l(X \backslash\{x\}) \leq \kappa$, de acordo com a Proposição 1.2.35.

Portanto, um compactum $X$ é um K-compactum se e somente se, para todo $x \in X, X \backslash\{x\}$ for linearmente Lindelöf. Por outro lado, é fácil ver que em um compactum $X, X \backslash\{x\}$ é Lindelöf se e somente se $\chi(x, X)=\omega$. Isso motiva a seguinte questão: em um compactum $X$, todo K-ponto tem caráter enumerável? Kunen [35] construiu em ZFC um compactum em que há um K-ponto com $\chi(x, X)>\omega$ (e $c f(\chi(x, X))=\omega)$. No entanto, a seguinte questão ainda está em aberto, de acordo com $[29]$ :

\section{Problema 2.4.6 Todo K-compactum tem caráter enumerável?}

De acordo com o Corolário 3.7 de [29], há um exemplo consistente de compactum $X \operatorname{com} \chi S(X)=\left\{\omega, \aleph_{\omega}\right\}$ (e portanto com $\chi(X)=\aleph_{\omega}$ ), sendo assim um exemplo de AB-compactum com caráter não enumerável. Esse exemplo não é um $\mathrm{K}$-compactum, pois $c S(X)=\left[\omega, \aleph_{\omega}\right]$.

Vejamos agora uma aplicação desses conceitos aos problemas de reflexão de funções cardinais. Se $\kappa$ é um cardinal e $\Upsilon$ é um conjunto de cardinais, dizemos que $\Upsilon$ omite $\kappa$ se $\kappa \notin \Upsilon$ e existe $\mu>\kappa \operatorname{com} \mu \in \Upsilon$. 
Proposição 2.4.7 Dado um cardinal infinito $\kappa$, se existir um espaço $X$ compacto $T_{2}$ tal que $\psi$ não reflita $\kappa^{+}$para $X$, então teremos exatamente uma das alternativas a seguir:

1. $c S(X) \cap R E G$ omite $\kappa^{+}$;

2. $\operatorname{Hus}(X) \leq \kappa^{+}<\psi(X)$.

Demonstração. Pelo Teorema 2.3.9, $\kappa^{+} \notin c S(X)$. Se existir algum cardinal regular $\mu>\kappa^{+}$tal que $\mu \in c S(X)$, então $c S(X) \cap R E G$ omite $\kappa^{+}$. Se não existir, não pode haver nenhum $A \subset X$, com $|A|$ regular $>\kappa$, e que convirja para um ponto $p \in X$. Ou seja, dado qualquer $p \in X, \operatorname{Hus}(p, X) \leq \kappa^{+}$. Além disso, pelo item (2) do Teorema 2.3.13, temos que $\psi(X)>\kappa^{+}$.

Corolário 2.4.8 Se existir um espaço $X$ compacto $T_{2}$ tal que $\psi$ não reflita $\omega_{1}$ para $X$, então teremos exatamente uma das alternativas a seguir:

1. $c S(X) \cap R E G$ omite $\omega_{1}$;

2. $X$ é um K-compactum com $\psi(X) \geq \aleph_{\omega}$.

A existência de um espaço satisfazendo o item (2) do corolário acima continua sendo um problema em aberto. Quanto a espaços $X$ compactos $T_{2}$ em que $c S(X) \cap$ $R E G$ omita algum cardinal regular, de acordo com [29] o único exemplo conhecido é um espaço construído em [28], que é a compactificação de Alexandroff de um espaço $T_{2}$ localmente compacto, de caráter enumerável, inicialmente $\omega_{1}$-compacto mas não compacto, de cardinalidade $\omega_{2}$. Chamando de $X$ essa compactificação, $\psi(X)=p s w(X)=|X|=\omega_{2}$, e $\chi S(X)=c S(X)=\left\{\omega, \omega_{2}\right\}$. Não conseguimos até o momento determinar se $\phi$ reflete $\omega_{1}$ para $X$, quando $\phi \in\{\psi, p s w\}$.

Corolário 2.4.9 Dado um cardinal infinito $\kappa$, se existir um espaço $X$ que seja um contra-exemplo consistente para o enunciado "psw reflete $\kappa^{+}$para a classe dos espaços compactos $T_{2}$ ", então teremos exatamente uma das alternativas a seguir:

1. $c S(X) \cap R E G$ omite $\kappa^{+}$;

2. Hus $(X) \leq \kappa^{+}<\psi(X) \leq p s w(X)$;

3. $\psi(X) \leq \kappa<p s w(X)$.

Nas alternativas (1) e (2), X será também um contra-exemplo consistente para o enunciado " $\psi$ reflete $\kappa^{+}$para a classe dos espaços compactos $T_{2}$ ". 


\subsection{Resultados com submodelos elementares}

O próximo resultado é uma generalização direta para cardinais quaisquer de um resultado não publicado de Alas e Junqueira, e de parte do Teorema 3.5 em [31], e apresentamos aqui para conveniência do leitor.

Lema 2.5.1 Seja $\phi \in\{\psi, \chi\}$. Se $M$ é um submodelo elementar $\kappa$-covering, com $\kappa \cup\{\kappa, X, \tau\} \subset M$ e $\phi\left(X_{M}\right) \leq \kappa$, então $\phi(X) \leq \kappa$.

Demonstração. $\mathbf{1}^{\circ}$ caso: $\phi=\psi$

Seja $x \in X \cap M$ arbitrário. Como $\psi\left(x, X_{M}\right) \leq \kappa$, existe $B \subset \tau \cap M$, com $|B| \leq \kappa$, tal que $\cap\{V \cap M: V \in B\}=\{x\}$. Como $M$ é $\kappa$-covering, existe $C \in M$, tal que $B \subset C$ e $|C| \leq \kappa$. Defina agora

$$
D=\{V \in C: V \in \tau \wedge x \in V\}
$$

$D \in M$ pois $C, \tau, x \in M$; temos ainda $B \subset D,|D| \leq \kappa, \cap\{V \cap M: V \in D\}=\{x\}$ e $D \subset M$.

Vamos agora mostrar que $M \models \psi(x, X) \leq \kappa$. Para isto vamos mostrar que $M \models \varphi(D, x, X, \tau, \kappa)$, onde $\varphi(D, x, X, \tau, \kappa)$ é a fórmula que expressa " $D$ é uma pseudobase para $x$ no espaço $(X, \tau)$, de cardinalidade $\leq \kappa$ ". Essa fórmula pode ser expressa como

$$
D \subset \tau \wedge \forall y(y=x \Leftrightarrow y \in \cap D) \wedge|D| \leq \kappa .
$$

Temos então que $\varphi^{M}(D, x, X, \tau, \kappa)$ seria expresso como

$$
(D \subset \tau)^{M} \wedge \forall y \in M(y=x \Leftrightarrow y \in \cap D)^{M} \wedge(|D| \leq \kappa)^{M}
$$

que por elementariedade é equivalente a

$$
D \subset \tau \wedge \forall y \in M(y=x \Leftrightarrow y \in \cap D) \wedge|D| \leq \kappa .
$$

E esta última expressão é claramente verdadeira, pelo que sabemos sobre $D$.

Agora, temos $M \models \psi(x, X) \leq \kappa$ para todo $x \in X \cap M$, ou seja,

$$
\forall x \in X \cap M(\psi(x, X) \leq \kappa)^{M},
$$

$\log 0$

$$
(\forall x \in X(\psi(x, X) \leq \kappa))^{M},
$$

ou seja,

$$
M \models \forall x \in X(\psi(x, X) \leq \kappa),
$$


e como $X, \kappa \in M$, temos que $\forall x \in X(\psi(x, X) \leq \kappa)$, logo $\psi(X) \leq \kappa$.

2 caso: $\phi=\chi$

Seja $x \in X \cap M$ arbitrário. Como $\chi\left(x, X_{M}\right) \leq \kappa$, existe $B \subset \tau \cap M$, com $|B| \leq \kappa$, tal que $x \in \cap B$, e se $U$ for uma vizinhança aberta de $x$ em $X_{M}$, existe $V_{U} \in B$ tal que $V_{U} \cap M \subset U$. Como $M$ é $\kappa$-covering, existe $C \in M$, tal que $B \subset C$ e $|C| \leq \kappa$. Defina agora

$$
D=\{V \in C: V \in \tau \wedge x \in V\}
$$

$D \in M$ pois $C, \tau, x \in M$; temos ainda que $B \subset D,|D| \leq \kappa, D \subset M, x \in \cap D$, e se $U$ for uma vizinhança aberta de $x$ em $X_{M}$, existe $V_{U} \in D$ tal que $V_{U} \cap M \subset U$.

Vamos agora mostrar que $M \models \chi(x, X) \leq \kappa$. Para isto vamos mostrar $M \models$ $\varphi(D, x, X, \tau, \kappa)$, onde $\varphi(D, x, X, \tau, \kappa)$ é a fórmula que expressa " $D$ é uma base para $x$ no espaço $(X, \tau)$, de cardinalidade $\leq \kappa$ ". Essa fórmula pode ser expressa como

$$
D \in[\tau]^{\leq \kappa} \wedge x \in \cap D \wedge \forall u \in \tau(x \in u \Rightarrow \exists v \in D(v \subset u)) .
$$

Temos então que $\varphi^{M}(D, x, X, \tau, \kappa)$ seria expresso como

$$
\left(D \in[\tau]^{\leq \kappa} \wedge x \in \cap D\right)^{M} \wedge \forall u \in \tau \cap M(x \in u \Rightarrow \exists v \in D \cap M(v \cap M \subset u \cap M)) .
$$

Como $D \subset M$, esta expressão é equivalente a

$$
\left(D \in[\tau]^{\leq \kappa} \wedge x \in \cap D\right)^{M} \wedge \forall u \in \tau \cap M(x \in u \Rightarrow \exists v \in D(v \cap M \subset u \cap M)) .
$$

E por sua vez, por elementariedade, esta expressão é equivalente a

$$
D \in[\tau]^{\leq \kappa} \wedge x \in \cap D \wedge \forall u \in \tau \cap M(x \in u \Rightarrow \exists v \in D(v \cap M \subset u \cap M)) .
$$

E esta última expressão é claramente verdadeira, pelo que sabemos sobre $D$. Agora, temos $M \models \chi(x, X) \leq \kappa$ para todo $x \in X \cap M$, ou seja,

$$
\forall x \in X \cap M(\chi(x, X) \leq \kappa)^{M}
$$

$\log 0$

$$
(\forall x \in X(\chi(x, X) \leq \kappa))^{M}
$$

ou seja,

$$
M \models \forall x \in X(\chi(x, X) \leq \kappa),
$$

e como $X, \kappa \in M$, temos que $\forall x \in X(\chi(x, X) \leq \kappa)$, $\log$ $\chi(X) \leq \kappa$.

O próximo lema generaliza para cardinais quaisquer o Teorema 2.5 em [30], com condições adicionais sobre o submodelo elementar. 
Lema 2.5.2 Sejam $(X, \tau)$ um espaço com $\chi(X) \leq \kappa^{+}$e $M$ um submodelo elementar com $\kappa^{+} \cup\left\{\kappa^{+}, X, \tau\right\} \subset M$. Então $X_{M}$ é subespaço de $X$.

Demonstração. Para obtermos nosso resultado, é suficiente mostrar que a topologia de $X_{M}$ é mais fina que a do subespaço $X \cap M$ e para isto basta mostrar que cada $x \in X \cap M$ possui uma base de vizinhanças abertas em $X \cap M$, cujos elementos também são abertos em $X_{M}$.

Dado um $x \in X \cap M$ arbitrário, como $\chi(X) \leq \kappa^{+}$, existe uma base $B_{x}$ de vizinhanças abertas de $x$ em $X$, com $\left|B_{x}\right| \leq \kappa^{+}$. Por Tarski podemos obter $B_{x} \in$ $M$, e como $\kappa^{+} \in M$ e $\kappa^{+} \subset M$, então $B_{x} \subset M$. Deste modo, $\left\{V \cap M: V \in B_{x}\right\}$ é base de vizinhanças em $X \cap M$ procurada.

Com estes lemas, podemos obter este resultado de reflexão:

Proposição 2.5.3 Seja $\phi \in\{\chi, \psi\}$. $\phi$ reflete $\kappa^{+}$para a classe dos espaços com caráter $\leq \kappa^{+}$.

Demonstração. O que precisamos mostrar é que, se $\phi(Y) \leq \kappa$ para todo $Y \in$ $[X]^{\leq \kappa^{+}}$, então $\phi(X) \leq \kappa$.

Usando o Lema 1.3.10, seja $M$ um submodelo elementar $\kappa$-covering, com $\kappa^{+} \cup$ $\left\{\kappa^{+}, X, \tau\right\} \subset M$ e $|M| \leq \kappa^{+}$.

Pelo Lema 2.5.2, $X_{M}$ é subespaço de $X$, e como $|X \cap M| \leq|M|=\kappa^{+}$, $\phi\left(X_{M}\right) \leq \kappa$; e pelo Lema 2.5.1 temos $\phi(X) \leq \kappa$.

Corolário 2.5.4 Se $\chi(Y) \leq \kappa$ para todo $Y \in[X]^{\leq \kappa^{+}}$, então $\chi(X) \neq \kappa^{+}$.

Corolário 2.5.5 Se $X$ é um espaço $T_{2}$ de "pointwise countable type", e se $\psi(Y) \leq$ $\kappa$ para todo $Y \in[X]^{\leq \kappa^{+}}$, então $\psi(X) \neq \kappa^{+}$.

Demonstração. Segue da Proposição 2.5.3 e do fato de que, se $X$ é um espaço de pointwise countable type, $\psi(X)=\chi(X)$.

Com o Corolário 2.5.4, obtemos uma relação entre reflexão e propriedade de Darboux, para o caráter:

Proposição 2.5.6 Dado qualquer cardinal infinito $\kappa$ e uma classe $S$ de espaços, se $\chi$ tiver a propriedade de Darboux em $\kappa^{+}$para $S$, então $\chi$ reflete $\kappa^{+}$para $S$. 
Demonstração. Seja $X$ um espaço da classe $S$. Precisamos mostrar que, se $\chi(Y) \leq$ $\kappa$ para todo $Y \in[X]^{\leq \kappa^{+}}$, então $\chi(X) \leq \kappa$.

Se tivéssemos $\chi(X)>\kappa$, pelo Corolário 2.5.4 teríamos $\chi(X)>\kappa^{+}$, e pela propriedade de Darboux, existe $Z \subset X$ tal que $\chi(Z)=\kappa^{+}$. Dado qualquer $Y \in$ $[Z]^{\leq \kappa^{+}} \subset[X]^{\leq \kappa^{+}}$, temos que $\chi(Y) \leq \kappa$. Logo, pelo Corolário 2.5.4, $\chi(Z) \neq \kappa^{+}$; contradição.

O caráter reflete todos os cardinais sucessores para a classe dos espaços compactos $T_{2}$ (resultado citado na Introdução, e que veremos novamente - Teorema 3.1.1). Em vista da proposição acima, é natural perguntar, se para todo cardinal infinito $\kappa, \chi$ tem a propriedade de Darboux em $\kappa^{+}$para a classe dos espaços compactos $T_{2}$. A resposta é que não podemos demonstrar isso em ZFC, pois de acordo com o Corolário 3.7 de [29], há um exemplo consistente de compactum $X$ com $\chi S(X)=\left\{\omega, \aleph_{\omega}\right\}$, e portanto $\chi(X)=\aleph_{\omega}$, mas não há nenhum $Y \subset X$ com $\chi(Y)=\omega_{1}$. Esse mesmo exemplo mostra que não vale a recíproca da proposição acima, mesmo restrita à classe dos espaços compactos $T_{2}$. 


\section{Capítulo 3}

\section{CH e reflexão do caráter}

\subsection{Introdução}

Continuaremos a estudar neste capítulo a propriedade de reflexão para a função cardinal $\chi$ (caráter), que é monótona. O resultado mais importante descrito na literatura é dado pelo Corolário 3.4 em [22]:

Teorema 3.1.1 $\chi$ reflete todos os cardinais infinitos para a classe dos espaços compactos $T_{2}$.

Em vista deste resultado, é natural pensar se é possível generalizá-lo para a classe dos espaços Lindelöf $T_{2}$. Resumiremos aqui uma discussão sobre este problema feita em [26] (demonstrações podem ser encontradas em [5]). Primeiro, note que o Teorema 3.1.1 implica que se $X$ for um espaço compacto $T_{2}$ e $\chi(Y)=\omega$ para todo $Y \in[X]^{\leq \omega_{1}}$, então $\psi(X)=\omega$. Este resultado pode ser estendido para espaços Lindelöf $T_{2}[26]$ :

Teorema 3.1.2 Se $X$ é um espaço Lindelöf $T_{2}$, com $\chi(Y)=\omega$ para todo $Y \in$ $[X]^{\leq \omega_{1}}$, então $\psi(X)=\omega$.

Este resultado não implica que o caráter reflita $\omega_{1}$ para os espaços Lindelöf $T_{2}$, pois neles podemos ter o caráter maior que o pseudocaráter. Temos no entanto os seguintes resultados [26] (que podem ser generalizados para cardinais maiores):

Teorema 3.1.3 CH é equivalente ao seguinte enunciado: " $\chi$ reflete $\omega_{1}$ para a classe dos espaços Lindelöf $T_{2} . "$. 
Teorema 3.1.4 Se $X$ é um espaço Lindelöf $T_{2}$, com $\chi(Y)=\omega$ para todo $Y \in$ $[X]^{\leq \omega_{1}}$, então $|X| \leq 2^{\omega}$.

O Teorema 3.1.4 estende o seguinte caso particular do Teorema de Arhangel'skiü: se $X$ é um espaço Lindelöf $T_{2}, \operatorname{com} \chi(X)=\omega$, então $|X| \leq 2^{\omega}$.

Há uma classe importante de problemas, relacionados à espaços linearmente Lindelöf [39][15], que são perguntas do tipo: "Sendo $P$ uma propriedade satisfeita por todos os espaços Lindelöf, então essa propriedade será satisfeita por todos os espaços linearmente Lindelöf? E nos espaços $\omega_{1}$-Lindelöf?". Podemos então considerar a propriedade de reflexão expressa no Teorema 3.1.3, e perguntar se é possível substituir no enunciado, "Lindelöf" por "linearmente Lindelöf", ou por " $\omega_{1}$-Lindelöf". O restante do capítulo irá responder a essa questão.

\subsection{Resultados auxiliares}

Nesta seção apresentaremos alguns lemas auxiliares que serão depois utilizados nos resultados de reflexão do caráter. O próximo lema é "folclore", e incluímos aqui para conveniência do leitor:

Lema 3.2.1 Sejam $X$ um espaço $T_{2}, D$ um subespaço denso de $X$ e $p \in D$. Então $\psi_{c}(p, X) \leq \chi(p, D)$.

Demonstração. Seja $\left\{V_{\alpha}: \alpha<\lambda\right\}$, onde $\lambda=\chi(p, D)$, uma família de vizinhanças abertas de $p$ em $X$ tal que $\left\{V_{\alpha} \cap D: \alpha<\lambda\right\}$ seja uma base de vizinhanças de $p$ no subespaço $D$.

Como $X$ é Hausdorff,

$$
\{p\}=\cap\{\bar{W}: W \text { aberto em } X, p \in W\} .
$$

Para cada $W$ como acima, existe $\beta<\lambda$ tal que

$$
W \cap D \supset V_{\beta} \cap D .
$$

Logo

$$
\bar{W}=\overline{W \cap D} \supset \overline{V_{\beta} \cap D}=\overline{V_{\beta}} .
$$

Portanto $\cap\left\{\overline{V_{\beta}}: \beta<\lambda\right\}=\{p\}$, o que conclui a demonstração.

O lema a seguir e sua demonstração é um resultado não publicado de $\mathrm{O}$. T. Alas: 
Lema 3.2.2 Sejam $X$ um $L(\kappa)$-espaço $T_{2}$ e $p \in X$ tais que $\psi_{c}(p, X)=\kappa$. Então existe $Y \in[X]^{\kappa}$ tal que $p \in Y$ e $\psi(p, Y)=\kappa$.

Demonstração. Seja $\left\{U_{\alpha}: \alpha<\kappa\right\}$ uma coleção de vizinhanças abertas de $p$ em $X$ tal que

$$
\cap\left\{\overline{U_{\alpha}}: \alpha<\kappa\right\}=\{p\} .
$$

Para cada $\alpha<\kappa$ defina $P_{\alpha}=\cap\left\{\overline{U_{\beta}}: \beta \leq \alpha\right\}$, e para cada $(\alpha, \beta) \in \kappa \times \kappa$ vamos escolher um ponto $p_{(\alpha, \beta)}$ em $X$ como a seguir:

$$
p_{(\alpha, \beta)} \in P_{\alpha} \backslash P_{\beta} \text { se } P_{\alpha} \backslash P_{\beta} \neq \emptyset
$$

e

$$
p_{(\alpha, \beta)}=p \text { caso contrário. }
$$

Defina

$$
Y=\left\{p_{(\alpha, \beta)}:(\alpha, \beta) \in \kappa \times \kappa\right\} ;
$$

então $|Y| \leq \kappa$ e $p \in Y$. Vamos mostrar que $\psi(p, Y)=\kappa$.

Suponha que não e seja $\lambda=\psi(p, Y)<\kappa$. Fixe $\left\{W_{\gamma}: \gamma<\lambda\right\}$ uma coleção de vizinhanças abertas de $p$ em $X$ tal que

$$
\cap\left\{W_{\gamma} \cap Y: \gamma<\lambda\right\}=\{p\} .
$$

Para cada $\gamma<\lambda,\left\{\overline{U_{\alpha}} \backslash W_{\gamma}: \alpha<\kappa\right\}$ é uma coleção de fechados com interseção vazia. Então existe algum $S_{\gamma} \subset \kappa$, com $\left|S_{\gamma}\right|<\kappa$, tal que

$$
\cap\left\{\overline{U_{\alpha}} \backslash W_{\gamma}: \alpha \in S_{\gamma}\right\}=\emptyset \text {. }
$$

Como $\left|\cup\left\{S_{\gamma}: \gamma<\lambda\right\}\right|<\kappa$, existe $\theta<\kappa$ tal que

$$
\cup\left\{S_{\gamma}: \gamma<\lambda\right\} \subset \theta
$$

Segue-se que $P_{\theta} \subset \cap\left\{W_{\gamma}: \gamma<\lambda\right\}$ e $P_{\theta} \cap Y=\{p\}$.

Fixe $\theta_{*}$ tal que $\theta<\theta_{*}<\kappa$ e $P_{\theta} \backslash P_{\theta_{*}} \neq \emptyset$; então $p_{\left(\theta, \theta_{*}\right)} \in\left(P_{\theta} \cap Y\right) \backslash\{p\}$, contradição.

No próximo lema, adaptamos para espaços $T_{2}$ um resultado de O. T. Alas, que era demonstrado para $L\left(\kappa^{+}\right)$-espaços regulares. Ou seja, nas hipóteses, enfraquecemos o axioma de separação, de regular para Hausdorff, ao custo de exigir que o espaço seja $\left[\kappa^{+}, 2^{\kappa}\right]$-compacto ao invés de $L\left(\kappa^{+}\right)$-espaço ou $\left[\kappa^{+}, \kappa^{+}\right]$-compacto. Tal adaptação foi necessária para obtermos na próxima seção os resultados de reflexão para espaços $T_{2}$. 
Lema 3.2.3 Sejam $\kappa$ um cardinal infinito, $X$ um espaço $T_{2}\left[\kappa^{+}, 2^{\kappa}\right]$-compacto $e$ $p \in X$ tais que $\psi_{c}(p, X) \geq \kappa^{+}$. Então:

1. $\exists S \in[X]^{<\kappa^{+}}$, com $p \in S$, tal que $\psi_{c}(\bar{S})>\kappa^{+}$; ou

2. $\exists Y \in[X]^{\kappa^{+}}$, com $p \in Y$, e um ponto $q \in Y$, com $t(q, Y)=\kappa^{+}$; ou

3. $\exists T \in[X]^{\kappa^{+}}$, com $p \in T$, tal que $\psi_{c}(T)=\psi(T)=\kappa^{+}$.

Demonstração. Vamos supor que (1) não ocorre, ou seja, que $\psi_{c}(\bar{S}) \leq \kappa^{+}$para todo $S \in[X]^{<\kappa^{+}} \operatorname{com} p \in S$.

Se houver algum $S \in[X]^{<\kappa^{+}}$, com $p \in S$, tal que $\psi_{c}(\bar{S})=\kappa^{+}$, então existe algum $y \in \bar{S}$ tal que $\psi_{c}(y, \bar{S})=\kappa^{+}$. Usando o Lema 3.2.2, obtemos então $T \in$ $[\bar{S}]^{\kappa^{+}} \subset[X]^{\kappa^{+}} \operatorname{com} y \in T$ e $\psi(y, T)=\kappa^{+}$. Logo $\psi(T)=\kappa^{+}$, ou seja, obtemos (3) (considerando-se $T \cup\{p\}$ ).

Podemos, portanto, a partir deste ponto assumir que $\psi_{c}(\bar{S}) \leq \kappa$ para todo $S \in[X]^{<\kappa^{+}} \operatorname{com} p \in S$.

Vamos agora construir por indução transfinita, para cada $\alpha \in \kappa^{+}$, um ponto $p_{\alpha} \in X$ e uma coleção $\mathcal{B}_{\alpha}$ de vizinhanças abertas de $p$, satisfazendo:

- $\left|\mathcal{B}_{\alpha}\right| \leq \kappa$,

- $\bigcup\left\{\mathcal{B}_{\beta}: \beta<\alpha\right\} \subset \mathcal{B}_{\alpha}$,

- $\left\{\bar{\Omega}: \Omega \in \mathcal{B}_{\alpha}\right\} \cap \overline{S_{\alpha}}=\{p\}$, onde $S_{\alpha}=\{p\} \cup\left\{p_{\beta}: \beta<\alpha\right\}$ e

- $p_{\alpha} \in \bigcap\left\{\bar{\Omega}: \Omega \in \mathcal{B}_{\alpha}\right\} \backslash \overline{S_{\alpha}}$.

Dado qualquer $\alpha \in \kappa^{+}$, note que $\psi_{c}\left(\overline{S_{\alpha}}\right) \leq \kappa$.

Defina $\mathcal{B}_{0}=\{X\}$ e $p_{0}$ como qualquer ponto de $X \backslash\{p\}\left(p_{0}\right.$ existe pois $\psi_{c}(p, X) \geq$ $\left.\kappa^{+}\right)$. Para cada $\alpha$ com $0<\alpha<\kappa^{+}$, defina $\mathcal{B}_{\alpha}$ e $p_{\alpha}$ do seguinte modo:

- Como $\psi\left(p, \overline{S_{\alpha}}\right) \leq \kappa$, existe uma coleção $\left\{O_{\lambda}: \lambda<\kappa\right\}$ de vizinhanças abertas de $p($ em $X)$ tal que

$$
\bigcap\left\{O_{\lambda}: \lambda<\kappa\right\} \cap \overline{S_{\alpha}}=\{p\}
$$

Para cada $\lambda<\kappa$, o fechado $\overline{S_{\alpha}} \backslash O_{\lambda}$ é $\left[\kappa^{+}, 2^{\kappa}\right]$-compacto, logo

$$
\overline{S_{\alpha}} \backslash\{p\}=\bigcup_{\lambda<\kappa} \overline{S_{\alpha}} \backslash O_{\lambda}
$$


também é.

Pelo Teorema 1.2.29(3) temos que

$$
\left|\overline{S_{\alpha}}\right| \leq 2^{\left|S_{\alpha}\right| \psi_{c}\left(\overline{S_{\alpha}}\right)} \leq 2^{\kappa}
$$

Para cada $z \in \overline{S_{\alpha}} \backslash\{p\}$, seja $A_{z}$ uma vizinhança aberta de $p$ em $X$ tal que $z \notin \overline{A_{z}}$. Desta forma,

$$
\left\{\overline{S_{\alpha}} \backslash \overline{A_{z}}: z \in \overline{S_{\alpha}} \backslash\{p\}\right\}
$$

é uma cobertura aberta de $\overline{S_{\alpha}} \backslash\{p\}$, de cardinalidade menor ou igual a $2^{\kappa}$. Como $\overline{S_{\alpha}} \backslash\{p\}$ é $\left[\kappa^{+}, 2^{\kappa}\right]$-compacto, existe um conjunto $\left\{z_{\gamma}: \gamma<\kappa\right\} \subset$ $\overline{S_{\alpha}} \backslash\{p\}$ tal que

$$
\overline{S_{\alpha}} \backslash\{p\}=\bigcup_{\gamma<\kappa} \overline{S_{\alpha}} \backslash \overline{A_{z_{\gamma}}}
$$

ou seja,

$$
\overline{S_{\alpha}} \cap \bigcap_{\gamma<\kappa} \overline{A_{z_{\gamma}}}=\{p\}
$$

Defina então

$$
\mathcal{B}_{\alpha}=\left\{A_{z_{\gamma}}: \gamma<\kappa\right\} \cup \bigcup_{\beta<\alpha} \mathcal{B}_{\beta} .
$$

Evidentemente, teremos $\left|\mathcal{B}_{\alpha}\right| \leq \kappa, \bigcup\left\{\mathcal{B}_{\beta}: \beta<\alpha\right\} \subset \mathcal{B}_{\alpha}$ e

$$
\bigcap\left\{\bar{\Omega}: \Omega \in \mathcal{B}_{\alpha}\right\} \cap \overline{S_{\alpha}}=\{p\} \text {. }
$$

- Escolha $p_{\alpha} \in \bigcap\left\{\bar{\Omega}: \Omega \in \mathcal{B}_{\alpha}\right\} \backslash \overline{S_{\alpha}}$. Note que $\bigcap\left\{\bar{\Omega}: \Omega \in \mathcal{B}_{\alpha}\right\} \backslash \overline{S_{\alpha}} \neq \emptyset$, pois caso contrário teríamos $\bigcap\left\{\bar{\Omega}: \Omega \in \mathcal{B}_{\alpha}\right\} \subset \overline{S_{\alpha}}$, e portanto $\bigcap\left\{\bar{\Omega}: \Omega \in \mathcal{B}_{\alpha}\right\}=$ $\{p\}$, contradizendo $\psi_{c}(p, X) \geq \kappa^{+}$.

Defina $\mathcal{B}=\bigcup_{\alpha<\kappa^{+}} \mathcal{B}_{\alpha}, Y=\{p\} \cup\left\{p_{\alpha}: \alpha<\kappa^{+}\right\}$e $Z=\bigcup\left\{\overline{S_{\alpha}}: \alpha<\kappa^{+}\right\}$. Temos que $|\mathcal{B}| \leq \kappa^{+},|Y|=\kappa^{+}, Y \subset Z \subset \bar{Y}$, e $\bigcap\{\bar{\Omega}: \Omega \in \mathcal{B}\} \cap Z=\{p\}$. Se $\bar{Y} \neq Z$, escolhendo qualquer $q \in \bar{Y} \backslash Z$, temos $t(q,\{q\} \cup Y)=\kappa^{+}$, ou seja, obtemos (2).

Podemos então assumir deste ponto em diante que $\bar{Y}=Z$. Note que $\psi_{c}(p, Z) \leq$ $\kappa^{+}$, pois $\overline{\Omega \cap Z} \subset \bar{\Omega} \cap Z$. Vamos mostrar que $\psi(p, Z)=\kappa^{+}$. Por contradição, suponha que $\psi(p, Z) \leq \kappa$. Então existe uma coleção $\left\{W_{\alpha}: \alpha<\kappa\right\}$ de vizinhanças abertas de $p$ tal que $\bigcap\left\{W_{\alpha}: \alpha<\kappa\right\} \cap Z=\{p\}$. Para cada $\alpha<\kappa$, temos

$$
\bigcap\left\{(\bar{\Omega} \cap Z) \backslash W_{\alpha}: \Omega \in \mathcal{B}\right\}=\emptyset,
$$

e como $X$ é $L\left(\kappa^{+}\right)$-espaço (por ser $\left[\kappa^{+}, 2^{\kappa}\right]$-compacto), existe $C_{\alpha} \in[\mathcal{B}]^{<\kappa^{+}}$tal que

$$
\bigcap\left\{\bar{\Omega} \cap Z: \Omega \in \mathcal{C}_{\alpha}\right\} \subset W_{\alpha} .
$$


Existe então algum $\delta \in \kappa^{+}$tal que $\bigcup\left\{\mathcal{C}_{\alpha}: \alpha<\kappa\right\} \subset \mathcal{B}_{\delta}$, logo

$$
\{p\}=\bigcap\left\{W_{\alpha}: \alpha<\kappa\right\} \cap Z \supset \bigcap\left\{\bar{\Omega} \cap Z: \Omega \in \mathcal{B}_{\delta}\right\} \supset\left\{p_{\delta}\right\},
$$

contradição, pois $p \neq p_{\delta}$.

Com isso temos $\psi_{c}(p, Z)=\kappa^{+}$. Usando o Lema 3.2.2 obtemos $T \in[Z]^{\kappa^{+}} \subset$ $[X]^{\kappa^{+}} \operatorname{com} p \in T$ e $\psi(p, T)=\kappa^{+}, \operatorname{logo} \psi(T)=\kappa^{+}$; ou seja, obtemos (3).

\subsection{Reflexão do caráter}

O primeiro teorema desta seção estende, quando $\kappa=\omega$, os Teoremas 3.1.2 e 3.1.4. A demonstração é em parte baseada em demonstrações de alguns resultados em [5]. Basicamente, aqui ao invés de exigirmos que o espaço tenha grau de Lindelöf $\leq \kappa$, exigimos apenas que ele seja $\left[\kappa^{+}, 2^{\kappa}\right]$-compacto.

Teorema 3.3.1 Se $X$ é um espaço $T_{2}\left[\kappa^{+}, 2^{\kappa}\right]$-compacto, com $\chi(Y) \leq \kappa$ para todo $Y \in[X]^{\leq \kappa^{+}}$, então $\psi_{c}(X) \leq \kappa, L(X) \leq \kappa e|X| \leq 2^{\kappa}$.

Demonstração. Vejamos primeiro que $\psi_{c}(X) \leq \kappa$. Suponha que exista $p \in X$ tal que $\psi_{c}(p, X) \geq \kappa^{+}$. Pelo Lema 3.2.3 vale um dos resultados (1), (2) ou (3) daquele enunciado. Claramente, (2) e (3) não podem ocorrer, pois para qualquer $Y \subset X$, $\psi(Y) \leq \psi_{c}(Y) \leq \chi(Y)\left(\psi_{c}(q, Y) \leq \chi(q, Y)\right.$ pelo Lema 3.2.1) e $t(Y) \leq \chi(Y)$. E o resultado (1) não pode ocorrer pois, usando o Lema 3.2.1, dado qualquer $S \in[X]^{<\kappa^{+}}$com $p \in S$, e qualquer $q \in \bar{S}$,

$$
\psi_{c}(q, \bar{S})=\psi_{c}(q, \overline{S \cup\{q\}}) \leq \chi(q, S \cup\{q\}) \leq \chi(S \cup\{q\}) \leq \kappa,
$$

pois $|S \cup\{q\}| \leq \kappa$. Logo, $\psi_{c}(\bar{S}) \leq \kappa$.

Defina, para cada $x \in X$, uma família $\left\{V_{\alpha, x}: \alpha \in \kappa\right\}$ de vizinhanças abertas de $x$ tal que $\bigcap_{\alpha \in \kappa} V_{\alpha, x}=\{x\}$.

Defina, por indução transfinita, uma família $\left\{A_{\lambda}: \lambda<\kappa^{+}\right\}$de subconjuntos de $X$, como a seguir:

- $A_{0} \in[X]^{\leq 2^{\kappa}}$

- se $\lambda$ for ordinal limite, defina $A_{\lambda}=\bigcup_{\xi<\lambda} A_{\xi}$ (note que $\left|A_{\xi}\right| \leq 2^{\kappa} \forall \xi<\lambda$ implica $\left.\left|A_{\lambda}\right| \leq 2^{\kappa}\right)$ 
- se $\lambda=\xi+1$, construa $A_{\lambda}$ como a seguir. Defina

$$
B_{\lambda}=\bigcup\left\{\bar{B}: B \in\left[A_{\xi}\right]^{\leq \kappa}\right\} .
$$

Dado qualquer $B \in\left[A_{\xi}\right]^{\leq \kappa}$, de acordo com o Lema 3.2.1 temos que, para todo $r \in \bar{B}$,

$$
\psi_{c}(r, \bar{B})=\psi_{c}(r, \overline{B \cup\{r\}}) \leq \chi(r, B \cup\{r\}) \leq \chi(B \cup\{r\}) \leq \kappa .
$$

Então pelo Teorema 1.2.29(3) temos que

$$
|\bar{B}| \leq 2^{|B| \psi_{c}(\bar{B})} \leq 2^{\kappa} .
$$

Deste modo, se $\left|A_{\xi}\right| \leq 2^{\kappa}$, então $\left|B_{\lambda}\right| \leq 2^{\kappa}$, pois $\left|\left[A_{\xi}\right]^{\leq \kappa}\right| \leq \kappa \cdot\left(2^{\kappa}\right)^{\kappa}=2^{\kappa}$. Defina

$$
\mathcal{C}_{\lambda}=\left[\left\{V_{\alpha, x}: \alpha \in \kappa, x \in A_{\xi}\right\}\right]^{\leq \kappa}
$$

$\mathrm{e}$

$$
\mathcal{S}_{\lambda}=\left\{C \in \mathcal{C}_{\lambda}: X \backslash \bigcup C \neq \emptyset\right\} .
$$

Para cada $C \in \mathcal{S}_{\lambda}$, escolha algum $x_{C} \in X \backslash \bigcup C$. Defina então $P_{\lambda}=$ $\left\{x_{C}: C \in \mathcal{S}_{\lambda}\right\}$. Note que $\left|P_{\lambda}\right| \leq 2^{\kappa}$ se $\left|A_{\xi}\right| \leq 2^{\kappa}$. Por fim, defina $A_{\lambda}=$ $A_{\xi} \cup B_{\lambda} \cup P_{\lambda}$. Note que $\left|A_{\lambda}\right| \leq 2^{\kappa}$ se $\left|A_{\xi}\right| \leq 2^{\kappa}$.

Defina agora $A=\bigcup_{\lambda<\kappa^{+}} A_{\lambda}$. Note que $|A| \leq 2^{\kappa}$, e queremos mostrar que $L(A) \leq \kappa$.

Vejamos que $\bar{B} \subset A$ para todo $B \in[A]^{\leq \kappa}$. Cada $b \in B$ pertence a algum $A_{\lambda_{b}}$ com $\lambda_{b}<\kappa^{+}$, e como $|B| \leq \kappa$, existe algum $\xi<\kappa^{+}$tal que $B \subset A_{\xi}$, logo $\bar{B} \subset B_{\xi+1} \subset A_{\xi+1} \subset A$.

Agora, vejamos que $F(X) \leq \kappa$. Seja $\left(x_{\xi}\right)_{\xi<\kappa^{+}}$uma sequência em $X$, de elementos dois a dois distintos (caso contrário não poderia ser uma sequência livre). Como $X$ é $L\left(\kappa^{+}\right)$-espaço (por ser $\left[\kappa^{+}, 2^{\kappa}\right]$-compacto), temos que

$$
\bigcap_{\eta<\kappa^{+}} \overline{\left\{x_{\xi}: \eta \leq \xi<\kappa^{+}\right\}} \neq \emptyset \text {. }
$$

Escolha então $x \in \bigcap_{\eta<\kappa^{+}} \overline{\left\{x_{\xi}: \eta \leq \xi<\kappa^{+}\right\}}$. Em particular, temos que $x \in \bar{E}$, onde $E=\left\{x_{\xi}: \xi<\kappa^{+}\right\}$. Como $|E \cup\{x\}|=\kappa^{+}, t(E \cup\{x\}) \leq \chi(E \cup\{x\}) \leq \kappa \mathrm{e}$ $x \in \bar{E}^{E \cup\{x\}}$, existe $\theta<\kappa^{+}$tal que $x \in \overline{\left\{x_{\xi}: \xi<\theta\right\}}$. Mas

$$
x \in \overline{\left\{x_{\xi}: \theta \leq \xi<\kappa^{+}\right\}},
$$


portanto a sequência $\left(x_{\xi}\right)_{\xi<\kappa^{+}}$não é livre.

Finalmente, vamos mostrar que $L(A) \leq \kappa$. Fazendo por absurdo, suponha que existe uma cobertura aberta $R$ de $A$ que não admite subcobertura de cardinalidade $\leq \kappa$. Vamos então construir, para cada $\eta<\kappa^{+}, x_{\eta} \in A$ e $R_{\eta} \subset R$, satisfazendo $\left|R_{\eta}\right| \leq \kappa$ e $\overline{\left\{x_{\xi}: \xi<\eta\right\}} \subset \bigcup R_{\eta}$, do seguinte modo:

- Defina $R_{0}=\emptyset$ e escolha qualquer $x_{0} \in A$

- Se $\eta=\xi+1$, escolha qualquer $V_{\eta} \in R$ tal que $x_{\xi} \in V_{\eta}$ e então defina $R_{\eta}=R_{\xi} \cup\left\{V_{\eta}\right\}$. É fácil ver que $\left|R_{\eta}\right| \leq \kappa$ e $\overline{\left\{x_{\xi}: \xi<\eta\right\}} \subset \cup R_{\eta}$. Depois escolha qualquer $x_{\eta} \in A \backslash \bigcup R_{\eta}$, que existe pois $\left|R_{\eta}\right| \leq \kappa$.

- Se $\eta$ for ordinal limite, como $X$ é $\left[\kappa^{+}, 2^{\kappa}\right]$-compacto, $\overline{\left\{x_{\xi}: \xi<\eta\right\}}$ também é. Como $\left|\left\{x_{\xi}: \xi<\eta\right\}\right| \leq \kappa$, temos que $\overline{\left\{x_{\xi}: \xi<\eta\right\}} \subset A$, logo

$$
\left|\overline{\left\{x_{\xi}: \xi<\eta\right\}}\right| \leq|A| \leq 2^{\kappa} \text {. }
$$

Portanto

$$
L\left(\overline{\left\{x_{\xi}: \xi<\eta\right\}}\right) \leq \kappa .
$$

Seja então $Q_{\eta} \in[R]^{\leq \kappa}$ tal que $\overline{\left\{x_{\xi}: \xi<\eta\right\}} \subset \bigcup Q_{\eta}$. Defina $R_{\eta}=Q_{\eta} \cup$ $\bigcup_{\xi<\eta} R_{\xi}$. Neste caso também é fácil ver que $\left|R_{\eta}\right| \leq \kappa$ e $\overline{\left\{x_{\xi}: \xi<\eta\right\}} \subset \bigcup R_{\eta}$. Escolha qualquer $x_{\eta} \in A \backslash \bigcup R_{\eta}$.

Agora, dado qualquer $\eta<\kappa^{+}$e qualquer $x \in \overline{\left\{x_{\xi}: \xi<\eta\right\}}$, temos que $x \in \bigcup R_{\eta}$, existe um aberto $O_{\eta}$ em $X$ tal que $\bigcup R_{\eta}=A \cap O_{\eta}$, e $\left\{x_{\xi}: \xi \geq \eta\right\} \cap O_{\eta}=\emptyset$, $\operatorname{logo} x \notin \overline{\left\{x_{\xi}: \xi \geq \eta\right\}}$. Deste modo, $\left(x_{\eta}\right)_{\eta<\kappa^{+}}$seria uma sequência livre em $X$, contradizendo o fato de que $F(X) \leq \kappa$.

Basta apenas mostrar então que $X=A$. Fazendo por absurdo, seja $y \in X \backslash A$. Para cada $x \in A$, seja $\alpha_{x} \in \kappa$ tal que $y \notin V_{\alpha_{x}, x}$. Deste modo, temos que

$$
A \subset \bigcup_{x \in A} V_{\alpha_{x}, x} \subset X \backslash\{y\}
$$

e como $L(A) \leq \kappa$, existe $T \in[A]^{\leq \kappa}$ tal que

$$
A \subset \bigcup_{x \in T} V_{\alpha_{x}, x} \subset X \backslash\{y\}
$$

Seja $\xi<\kappa^{+}$tal que $T \subset A_{\xi}$. Definindo $C=\left\{V_{\alpha_{x}, x}: x \in T\right\}$, temos que $C \in S_{\xi+1}$, $\operatorname{logo} x_{C} \in A_{\xi+1} \subset A$. Ao mesmo tempo $x_{C} \in X \backslash \bigcup C \subset X \backslash A$, contradição. 
Defina, para espaços $T_{2}$, a seguinte função cardinal $\phi: \phi(X)$ será o menor cardinal infinito $\kappa$ tal que $X$ seja $\left[\kappa^{+}, 2^{\kappa}\right]$-compacto e $\chi(Y) \leq \kappa$ para todo $Y \in$ $[X]^{\leq \kappa^{+}}$. Pelo Teorema 3.3.1, $|X| \leq 2^{\phi(X)}$. Além disso é fácil ver que sempre teremos $\phi(X) \leq L(X) \chi(X)$. Logo o Teorema 3.3.1 estende o Teorema de Arhangel'skiı̌ $|X| \leq \overline{2}^{L(X) \chi(X)}$.

Caso conseguíssemos, no caso $\kappa=\omega$ do Teorema 3.3.1, trocar a hipótese do espaço ser $\left[\omega_{1}, 2^{\omega}\right]$-compacto por $\omega_{1}$-Lindelöf, responderíamos de forma afirmativa a seguinte questão colocada em [6]:

Problema 3.3.2 Se $X$ for um espaço $\omega_{1}$-Lindelöf $T_{2}$ de caráter enumerável, $|X| \leq$ $2^{\omega}$ ?

No resultado a seguir, generalizamos para todos os cardinais, um espaço construído na demonstração do Teorema 3.4.4 em [5]. Para tornar mais natural essa generalização, escolhemos trabalhar com Cubos de Cantor. Assim sendo, no caso $\kappa=\omega$, substituímos um intervalo da reta real por um Conjunto de Cantor, mas sem perder as propriedades desejadas.

Proposição 3.3.3 $\left(2^{\kappa}>\kappa^{+}\right)$Existe um espaço $X, T_{2}$, com $L(X) \leq \kappa$, onde $\chi(Y) \leq \kappa$ para todo $Y \in[X]^{\leq \kappa^{+}}$, mas $\chi(X) \geq \kappa^{+}$.

Demonstração. Escolha qualquer $p$ do cubo de Cantor $2^{\kappa}$, e defina

$$
X=\left(\left(2^{\kappa} \backslash\{p\}\right) \times\{0\}\right) \cup\left(2^{\kappa} \times\{1\}\right),
$$

com a seguinte topologia:

- os pontos em $\left(2^{\kappa} \backslash\{p\}\right) \times\{1\}$ são isolados,

- se $A$ é uma vizinhança aberta de $q \neq p$ no cubo de Cantor $2^{\kappa}$, então

$$
((A \backslash\{p\}) \times\{0\}) \cup((A \backslash\{p, q\}) \times\{1\})
$$

é uma vizinhança aberta de $(q, 0)$ em $X$,

- se $A$ é uma vizinhança aberta de $p$ no cubo de Cantor $2^{\kappa}$, e se

$$
Z \in\left[\left(2^{\kappa} \backslash\{p\}\right) \times\{1\}\right]^{\leq \kappa^{+}},
$$

então $(A \times\{1\}) \backslash Z$ é uma vizinhança aberta de $(p, 1)$ em $X$. 
Vejamos agora que o subespaço $S=2^{\kappa} \backslash\{p\}$ do cubo de Cantor $2^{\kappa}$ satisfaz $L(S) \leq k$. Para cada $\alpha \in \kappa$, defina $F_{\alpha}=\left\{x \in 2^{\kappa}: \pi_{\alpha}(x) \neq \pi_{\alpha}(p)\right\}$. Basta agora notar que cada $F_{\alpha}$ é fechado em $2^{\kappa}$, logo compacto, e que $S=\bigcup_{\alpha \in \kappa} F_{\alpha}$.

Como a topologia de $\left(2^{\kappa} \backslash\{p\}\right) \times\{0\}$ como subespaço de $X$ é a mesma da topologia como subespaço de $2^{\kappa} \times\{0\}$, então qualquer cobertura aberta de $X$ tem uma subcoleção de cardinalidade $\leq \kappa$ que cobre $\left(2^{\kappa} \backslash\{p\}\right) \times\{0\}$, e deixa de cobrir no máximo $\kappa$ pontos de $\left(2^{\kappa} \backslash\{p\}\right) \times\{1\}$. Logo, $L(X) \leq k$.

Dado qualquer $Y \subset X$, é evidente que se $x \in Y \cap\left(\left(2^{\kappa} \backslash\{p\}\right) \times\{1\}\right)$, então $\chi(x, Y) \leq \kappa$; se $x \in Y \cap\left(\left(2^{\kappa} \backslash\{p\}\right) \times\{0\}\right)$, então $\chi(x, Y) \leq \kappa$ pois $\chi\left(2^{\kappa}\right)=\kappa$. Se $|Y| \leq \kappa^{+}$e $(p, 1) \in Y$, então $(p, 1)$ é ponto isolado em $Y, \operatorname{logo} \chi(Y) \leq \kappa$.

Por fim, vejamos que $\chi((p, 1), X) \geq \kappa^{+}$. Fazendo por absurdo, suponha que $\left\{A_{\alpha}: \alpha \in \kappa\right\}$ seja uma base de vizinhanças abertas de $(p, 1)$ em $X$. Para cada $\alpha \in \kappa$, escolha algum $x_{\alpha} \in A_{\alpha} \backslash\{(p, 1)\}$ (existe sob a condição $2^{\kappa}>\kappa^{+}$, pois todo aberto básico em $2^{\kappa}$ tem cardinalidade igual a $\left.2^{\kappa}\right)$. Considere agora $V$ uma vizinhança aberta qualquer de $(p, 1)$ em $X$, e defina $U=V \backslash\left\{x_{\alpha}: \alpha \in \kappa\right\}$. É imediato que $U$ também é uma vizinhança aberta de $(p, 1)$ em $X$. Mas dado qualquer $\alpha \in \kappa$, temos que $A_{\alpha} \nsubseteq U$, contradição.

O espaço construído no exemplo acima não é regular, quando $2^{\kappa}>\kappa^{+}$. De fato, sejam $V=(A \times\{1\}) \backslash Z$ uma vizinhança básica de $(p, 1)$, como descrito acima, e algum $z \in V \backslash\{(p, 1)\}$. Temos que $z=(q, 1)$ para algum $q \in 2^{\kappa} \backslash\{p\}$. Se $U$ for uma vizinhança básica de $(q, 0)$, temos que $|U \cap(A \times\{1\})|=2^{\kappa}$, logo $U \cap V \neq \emptyset$ e portanto $(q, 0) \in \bar{V}$. Mostramos assim que $(p, 1)$ não tem nenhuma vizinhança aberta $V$ tal que $\bar{V} \subset\left(2^{\kappa} \times\{1\}\right)$, logo $X$ não é regular. Não sabemos se este exemplo pode ser modificado de modo a se obter a regularidade.

De acordo com o realizado neste capítulo, podemos agora obter os seguintes resultados.

Teorema 3.3.4 Para qualquer cardinal infinito $\kappa$, são equivalentes, em ZFC:

i) $2^{\kappa}=\kappa^{+}$;

ii) $\chi$ reflete $\kappa^{+}$para a classe dos $L\left(\kappa^{+}\right)$-espaços $T_{2}$;

iii) $\chi$ reflete $\kappa^{+}$para a classe $\left\{X: X\right.$ é $T_{2}$ e $\left.l l(X) \leq \kappa\right\}$;

iv) $\chi$ reflete $\kappa^{+}$para a classe $\left\{X: X\right.$ é $T_{2}$ e $\left.L(X) \leq \kappa\right\}$.

Demonstração. (i) $\Rightarrow$ (ii): Vamos mostrar, sob (i), que se $X$ é um $L\left(\kappa^{+}\right)$-espaço $T_{2}$, e $\chi(Y) \leq \kappa$ para todo $Y \in[X]^{\leq \kappa^{+}}$, então $\chi(X) \leq \kappa$. Como $2^{\kappa}=\kappa^{+}, X$ é $\left[\kappa^{+}, 2^{\kappa}\right]$-compacto, logo pelo Teorema 3.3.1, $|X| \leq 2^{\kappa}=\kappa^{+}$, e portanto $\chi(X) \leq \kappa$.

(ii) $\Rightarrow$ (iii): Se $l l(X) \leq \kappa$, então $X$ é $L\left(\kappa^{+}\right)$-espaço, de acordo com o Corolário 1.2.34. 
(iii) $\Rightarrow($ iv $): l l(X) \leq L(X)$.

(iv) $\Rightarrow($ i): Contra-positiva da Proposição 3.3.3.

Corolário 3.3.5 São equivalentes em ZFC:

i) $\mathrm{CH}$;

ii) $\chi$ reflete $\omega_{1}$ para a classe dos $L\left(\omega_{1}\right)$-espaços $T_{2}$;

iii) $\chi$ reflete $\omega_{1}$ para a classe dos espaços $T_{2}$ linearmente Lindelöf;

iv) $\chi$ reflete $\omega_{1}$ para a classe dos espaços $T_{2}$ Lindelöf.

Este último corolário estende portanto, o Teorema 3.1.3, e responde à questão apresentada no início deste capítulo. 


\section{Capítulo 4}

\section{Funções globais}

\subsection{Introdução}

Neste capítulo estudaremos a propriedade de reflexão em funções cardinais globais como $d$ (densidade), $L$ (grau de Lindelöf) e $l l$ (grau de Lindelöf linear).

Os principais resultados de reflexão para $L$ e $d$ conhecidos anteriormente são os seguintes (extraídos dos Teoremas 2.3, 2.4 e 2.5 de [22], e Teorema 3 de [19]):

\section{Teorema 4.1.1}

1. L reflete todo cardinal sucessor;

2. L reflete todo cardinal limite forte e singular para a classe dos espaços $T_{2}$;

3. (GCH $+\#$ cardinais inacessiveis) $L$ reflete todos os cardinais para a classe dos espaços $T_{2}$;

4. se $\kappa$ for um cardinal singular, existe um contra-exemplo para a afirmação " $L$ tem a propriedade de Darboux em $\kappa$ para a classe dos espaços $T_{1}$ ".

\section{Teorema 4.1.2}

1. Para todo cardinal $\kappa, d$ reflete $\kappa$ se e somente se $d$ tem a propriedade de Darboux em $\kappa$;

2. $d$ reflete todo cardinal regular;

3. $d$ reflete todo cardinal limite forte para a classe dos espaços $T_{2}$; 
4. $(G C H) d$ reflete todos os cardinais para a classe dos espaços $T_{2}$;

5. se $\kappa$ for um cardinal singular, existe um contra-exemplo para a afirmação "d reflete $\kappa$ para a classe dos espaços $T_{1} "$;

6. d reflete todo cardinal de cofinalidade enumerável para a classe dos espaços $T_{2}$.

Considerando os teoremas acima, bem como uma certa similaridade entre os resultados para $L$ e para $d$, surgem naturalmente algumas questões como as seguintes:

Problema 4.1.3 L reflete os cardinais fortemente inacessiveis para a classe de todos os espaços topológicos?

Problema 4.1.4 L reflete os cardinais fracamente inacessiveis para a classe de todos os espaços topológicos?

Problema 4.1.5 $d$ reflete os cardinais singulares para a classe dos espaços $T_{2}$ ?

Nas próximas seções deste capítulo, iremos apresentar respostas completas ou parciais para essas questões. Na última seção apresentaremos um resultado de reflexão para $l l$.

\subsection{Resultados relacionados ao spread}

Alguns resultados de reflexão para $L$ ou $d$, podem ser obtidos usando a função cardinal $s$ (spread). Se $\phi \in\{L, d\}$, e $\kappa$ for um cardinal infinito menor que $\widehat{s}(X)$, então $\phi$ reflete $\kappa$ para $X$, pois existe um discreto $Y \in[X]^{\kappa}$, e $\phi(Y)=\kappa$.

Resultados como os do parágrafo anterior estão relacionados a um problema muito importante da Topologia Geral, que é o de quando o spread é atingido, ou seja, quando um espaço $X$ possui um subespaço discreto de cardinalidade $s(X)$. Esse e outros problemas similares (para funções cardinais definidas por meio de algum sup) são conhecidos na literatura como "problemas sup = max" (ver [21]). Quando o spread é atingido, temos $\widehat{s}(X)=(s(X))^{+}$, caso contrário temos $\widehat{s}(X)=$ $s(X)$.

Para o Problema 4.1.5, obtivemos algumas respostas parciais. Na proposição que segue, um espaço é $d$-separável se tiver algum subespaço denso que seja união enumerável de discretos [27]. 
Proposição 4.2.1 Seja $\kappa$ um cardinal singular. Teremos então que:

1. $d$ reflete $\kappa$ para a classe dos espaços compactos $T_{2}$;

2. $d$ reflete $\kappa$ para a classe dos espaços d-separáveis.

Demonstração. Se $X$ é compacto $T_{2}$, de acordo com [25] temos

$$
d(X) \leq s(X) \cdot \widehat{F}(X),
$$

$\operatorname{logo} d(X) \leq \widehat{s}(X)$. Considerando o item (1) do Teorema 4.1.2, vamos mostrar que $d$ tem a Propriedade de Darboux em $\kappa$ para $X$. Suponha que $d(X)>\kappa$. Então $\kappa<\widehat{s}(X)$, e portanto existe um discreto $Y \in[X]^{\kappa}, \operatorname{com} d(Y)=\kappa$.

Se $X$ for $d$-separável, existe $Y \subset X$, com $\bar{Y}=X$ e $Y=\bigcup_{n \in \omega} D_{n}$, onde cada $D_{n}$ é discreto. Se $d(X)<\aleph_{\omega}$ o resultado é imediato, caso contrário temos que

$$
d(X) \leq|Y|=\sup _{n \in \omega}\left|D_{n}\right| \leq s(X) \leq \widehat{s}(X),
$$

e podemos então proceder como no parágrafo anterior.

Em [21] são apresentados os seguintes resultados mostrando quando o spread atinge ou não um cardinal limite forte:

Teorema 4.2.2 Sejam $X$ um espaço $T_{2}$ e $\kappa$ um cardinal limite forte tais que $s(X)=\kappa$. Então:

1. se $\kappa$ for singular, o spread é atingido;

2. se $\kappa$ for um cardinal "fracamente compacto", o spread é atingido;

3. se $\kappa$ for um cardinal fortemente inacessivel que não seja "fracamente compacto", o spread nem sempre é atingido. Mais precisamente, haverá um exemplo consistente de espaço linearmente ordenado $X$, com $s(X)=\widehat{s}(X)=$ $\kappa$.

A relevância do teorema acima para a reflexão de $L$ se torna clara com a próxima proposição.

Proposição 4.2.3 Seja $\kappa$ um cardinal limite forte onde o spread é atingido para todo espaço $X$ com $s(X)=\kappa$. Então L reflete $\kappa$ para a classe dos espaços $T_{2}$. 
Demonstração. Seja $\kappa$ um cardinal como do enunciado e suponha que $L(X) \geq \kappa$. Como $L(X) \leq|X| \leq 2^{2^{s(X)}}$ (desigualdade de Hajnal-Juhász), temos que $s(X) \geq$ $\kappa$, pois $\kappa$ é limite forte. Se $s(X)>\kappa$, então $\widehat{s}(X)>\kappa$; se $s(X)=\kappa$, como o spread é atingido, então $\widehat{s}(X)=\kappa^{+}$. Em qualquer caso, existe um subespaço $Y$ discreto, com $|Y|=\kappa$, e portanto $L(Y)=\kappa$.

Corolário 4.2.4 L reflete todo cardinal limite forte e singular, e todo cardinal fracamente compacto, para a classe dos espaços $T_{2}$.

Todo cardinal fracamente compacto é fortemente inacessível, mas a recíproca não é verdadeira. Resta então saber o que ocorre com os cardinais fortemente inacessíveis que não são fracamente compactos. Neste caso, considerando o item (3) do Teorema 4.2.2, não poderemos responder a essa questão usando a Proposição 4.2.3. Portanto, uma eventual resposta afirmativa ao Problema 4.1.3 dependerá de alguma demonstração de outra natureza, o que buscaremos na próxima seção.

\subsection{L e cardinais fortemente inacessíveis}

Introduziremos a seguir um novo conceito que consideramos útil para compreender com mais profundidade a função cardinal $L$.

Definição 4.3.1 Seja $X$ um espaço topológico. Para cada cobertura aberta $C$ de $X$ defina

$$
m(C)=\min \{|S|: S \text { é subcobertura de } C\} .
$$

Defina então

$$
\begin{gathered}
l S(X)=\{m(C): C \text { é cobertura aberta de } X\} \text { e } \\
\overline{l S}(X)=\{\lambda: \lambda \leq \mu \text { para algum } \mu \in l S(X)\} .
\end{gathered}
$$

O conjunto de cardinais $l S(X)$ é uma espécie de "espectro" de cardinais relacionado às coberturas abertas "minimais" do espaço. É fácil ver que

$$
L(X)=\omega+\sup l S(X)=\omega+\sup \overline{l S}(X),
$$

que há sempre cardinais finitos em $l S(X)$ (pois $1 \in l S(X)$ ), e que $X$ é compacto se e somente se todo elemento de $l S(X)$ for finito. O espectro $l S(X)$ nos dá mais 
informações sobre $X$ do que apenas o valor de $L(X)$, e isso se tornará mais claro ao longo do capítulo.

Como trivialmente $L$ reflete todos os cardinais infinitos para a classe dos espaços compactos, deste momento em diante, sempre que escolhermos um cardinal em $l S(\cdot)$ ou $\overline{l S}(\cdot)$, este será um cardinal infinito.

Proposição 4.3.2 Se $\lambda \in \bar{l}(X)$ então $X$ tem um subespaço $Y$ separado à direita com $|Y|=\lambda$. Se além disso, $\lambda$ for regular, então teremos ainda que

$$
L(Y)=l l(Y)=\lambda \text {. }
$$

Demonstração. Seja $C$ uma cobertura aberta de $X$ tal que $m(C) \geq \lambda$. Definiremos uma sequência $\left\{p_{\alpha}: \alpha<\lambda\right\}$ de pontos de $X$ e uma sequência $\left\{A_{\alpha}: \alpha<\lambda\right\}$ de elementos da cobertura $C$ como a seguir: para cada $\alpha<\lambda$, supondo já definidos $\left\{p_{\beta}: \beta<\alpha\right\}$ e $\left\{A_{\beta}: \beta<\alpha\right\}$, como $|\alpha|<\lambda \leq m(C)$, podemos escolher um ponto $p_{\alpha} \in X \backslash \bigcup_{\beta<\alpha} A_{\beta}$, e depois escolher qualquer $A_{\alpha} \in C$ tal que $p_{\alpha} \in A_{\alpha}$.

É fácil ver que $Y=\left\{p_{\alpha}: \alpha<\lambda\right\}$ é separado à direita e que $L(Y)=l l(Y)=\lambda$ se $\lambda$ for regular.

A primeira aplicação desta proposição é o seguinte teorema, que afirma algo que já sabíamos sobre reflexão, mas nos diz algo novo sobre Propriedade de Darboux de $L$.

\section{Teorema 4.3.3}

i) L reflete todos os cardinais sucessores;

ii) L tem a propriedade de Darboux em todos os cardinais regulares.

Demonstração. i) Se $\lambda$ é um cardinal infinito e $L(X) \geq \lambda^{+}$, então $\lambda^{+} \in \overline{l S}(X)$, logo pela Proposição 4.3.2 existe $Y \in[X]^{\lambda^{+}} \operatorname{com} L(Y)=\lambda^{+}$.

ii) Se $\kappa$ é um cardinal regular e $L(X)>\kappa$, teremos $\kappa \in \overline{l S}(X)$, e consequentemente $Y \in[X]^{\kappa} \operatorname{com} L(Y)=\kappa$.

Lembrando que $L(X)=\omega+\sup l S(X)$, o próximo resultado mostra que a reflexão dos cardinais regulares é facilmente obtida quando este sup é atingido.

Teorema 4.3.4 Sejam $\kappa$ um cardinal fracamente inacessivel e $X$ um espaço topológico. Se $L(X) \neq \kappa$ ou $\kappa \in l S(X)$, então $L$ reflete $\kappa$ para $X$. 
Demonstração. Se $L(X)<\kappa$, o resultado é trivial, pela definição de reflexão. Se $L(X)>\kappa$ ou $\kappa \in l S(X)$, então $\kappa \in \overline{l S}(X)$ e teremos o resultado pela Proposição 4.3.2.

Para os próximos resultados, precisaremos de alguns elementos de aritmética cardinal, como cofinalidades de ordens parciais, cardinalidade de coberturas e resultados da Teoria PCF de Shelah. Uma referência bastante abrangente é [1], onde encontramos as seguintes definições.

Definição 4.3.5 Dados cardinais $\mu, \chi$ e $\lambda$, com $\mu \geq \chi \geq \lambda \geq \omega, \operatorname{cov}(\mu, \chi, \lambda)$ é $a$ menor cardinalidade de algum $X \subset[\mu]^{<\chi}$ tal que, dado qualquer $a \in[\mu]^{<\lambda}$, existe $b \in X \operatorname{com} a \subset b$.

Definição 4.3.6 Dados cardinais $\mu$ e $\lambda, \operatorname{com} \mu>\lambda \geq \omega, \operatorname{cf}\left([\mu]^{\lambda}, \subset\right)$ é a cofinalidade da ordem parcial $\left([\mu]^{\lambda}, \subset\right)$, que é a menor cardinalidade de algum $X \subset[\mu]^{\lambda}$ tal que, dado qualquer $a \in[\mu]^{\lambda}$, existe $b \in X$ com $a \subset b$.

A partir das definições acima, é fácil ver que $\operatorname{cf}\left([\mu]^{\lambda}, \subset\right)=\operatorname{cov}\left(\mu, \lambda^{+}, \lambda^{+}\right)$.

Enunciaremos algumas propriedades de $\operatorname{cov}(\mu, \chi, \lambda)$ e $c f\left([\mu]^{\lambda}, \subset\right)$ que usaremos nesta e nas próximas seções.

Proposição 4.3.7 [1] Dados cardinais $\mu$ e $\lambda$, com $\mu>\lambda \geq \omega$,

$$
\mu^{\lambda}=\left|[\mu]^{\lambda}\right|=2^{\lambda} \cdot \operatorname{cf}\left([\mu]^{\lambda}, \subset\right) .
$$

Proposição 4.3.8 Se $\lambda$ for um cardinal singular e limite forte, então

$$
2^{\lambda}=c f\left([\lambda]^{c f(\lambda)}, \subset\right) .
$$

Demonstração. Denotando por I a função gimel, como as potências abaixo de $\lambda$ não se tornam eventualmente constantes, temos que

$$
2^{\lambda}=\beth\left(2^{<\lambda}\right)=\beth(\lambda)=\lambda^{c f(\lambda)}=2^{c f(\lambda)} \cdot c f\left([\lambda]^{c f(\lambda)}, \subset\right) .
$$

Mas $2^{c f(\lambda)}<\lambda$ enquanto que $2^{\lambda}>\lambda$, logo

$$
2^{\lambda}=c f\left([\lambda]^{c f(\lambda)}, \subset\right) .
$$


As próximas proposições apresentam propriedades básicas (enunciadas mas não demonstradas em [41] e [37]) sobre $\operatorname{cov}(\mu, \chi, \lambda)$ que utilizaremos frequentemente. As demonstrações podem ser encontradas no Apêndice A.

Proposição 4.3.9 Sejam $\mu, \chi, \lambda, \mu^{\prime}, \chi^{\prime}, \lambda^{\prime}$ cardinais infinitos, com $\mu^{\prime} \geq \mu \geq \chi^{\prime} \geq$ $\chi \geq \lambda^{\prime} \geq \lambda$. Temos então:

1. $\operatorname{cov}(\mu, \chi, \lambda) \leq \operatorname{cov}\left(\mu^{\prime}, \chi, \lambda\right)$;

2. $\operatorname{cov}(\mu, \chi, \lambda) \geq \operatorname{cov}\left(\mu, \chi^{\prime}, \lambda\right)$;

3. $\operatorname{cov}(\mu, \chi, \lambda) \leq \operatorname{cov}\left(\mu, \chi, \lambda^{\prime}\right)$.

Proposição 4.3.10 Sejam $\mu, \chi, \lambda$ cardinais infinitos, com $\mu \geq \chi \geq \lambda$. Se $\mu$ for regular ou $\mu>\chi$, então $\operatorname{cov}(\mu, \chi, \lambda) \geq \mu$.

Proposição 4.3.11 Sejam $\mu$ e $\lambda$ cardinais infinitos, com $\lambda \leq c f(\mu)$. Então $\operatorname{cov}(\mu, \mu, \lambda) \leq c f(\mu)$.

Os dois próximos teoremas estão entre os principais resultados deste trabalho, e poderemos responder de forma afirmativa o Problema 4.1.3. Parte do argumento a seguir é baseado na demonstração do Teorema 3.5 (c) em [31].

Teorema 4.3.12 Sejam $\kappa$ um cardinal fracamente inacessivel e $(X, \tau)$ um espaço topológico. Para que $L$ reflita $\kappa$ para $X$, é suficiente que tenhamos a seguinte condição: para todo $\lambda<\kappa$, existe algum $\mu \in l S(X)$ tal que $\mu>\lambda$ e $\operatorname{cov}\left(\mu, \mu, \lambda^{+}\right) \leq$ $\kappa$.

Demonstração. Para cada $\lambda<\kappa$ e o correspondente $\mu$ dado pela hipótese, seja $C_{\lambda}$ uma cobertura aberta de $X$, com

$$
\left|C_{\lambda}\right|=m\left(C_{\lambda}\right)=\mu \text {. }
$$

Além disso, como $\operatorname{cov}\left(\mu, \mu, \lambda^{+}\right) \leq \kappa$, podemos fixar $Q_{\lambda} \subset\left[C_{\lambda}\right]^{<\left|C_{\lambda}\right|}$, com $\left|Q_{\lambda}\right| \leq \kappa$, tal que dado qualquer $S \in\left[C_{\lambda}\right]^{\leq \lambda}$, existe algum $T \in Q_{\lambda}$ tal que $S \subset T$.

Seja agora $M \prec H_{\theta}$ dado pelo Teorema 1.3.5, $\operatorname{com} A \subset M$ e $|M|=|A|=\kappa$, onde

$$
A=\{X, \tau\} \cup \kappa \cup\{\kappa\} \cup\left\{C_{\lambda}: \lambda<\kappa\right\} \cup\left\{Q_{\lambda}: \lambda<\kappa\right\} .
$$

Note que $Q_{\lambda} \subset M$ para todo $\lambda<\kappa$, pois $\kappa \in M$ e $\kappa \subset M$. Evidentemente $|X \cap M| \leq \kappa$. Vamos provar que $L(X \cap M) \geq \kappa$ mostrando que, para todo $\lambda<\kappa$, 
$L\left(X_{M}\right)>\lambda$, o que implica que $L(X \cap M)>\lambda$ (note que $L(X \cap M) \geq L\left(X_{M}\right)$ pois a topologia de $X \cap M$ é mais fina que a de $\left.X_{M}\right)$.

Para cada $\lambda<\kappa$, o fato de que $C_{\lambda}$ é uma cobertura aberta de $X$, com $m\left(C_{\lambda}\right)=$ $\left|C_{\lambda}\right|$, pode ser expresso por uma fórmula $\varphi\left(C_{\lambda}, X, \tau\right)$, que por sua vez pode ser expandida como

$$
C_{\lambda} \subset \tau \wedge \varphi_{1}\left(C_{\lambda}, X\right) \wedge \varphi_{2}\left(C_{\lambda}, X\right),
$$

onde $\varphi_{1}\left(C_{\lambda}, X\right)$ significa " $C_{\lambda}$ cobre $X$ ", e pode ser explicitado como

$$
\forall x \in X\left(\exists V \in C_{\lambda}(x \in V)\right),
$$

e $\varphi_{2}\left(C_{\lambda}, X\right)$ significa " $C_{\lambda}$ não tem subcobertura de cardinalidade menor", e pode ser explicitado como

$$
\forall E\left(E \in\left[C_{\lambda}\right]^{<\left|C_{\lambda}\right|} \Rightarrow \exists x \in X(x \notin \cup E)\right) .
$$

Agora, como $\left\{C_{\lambda}, X\right\} \subset M$, temos que por elementariedade valem $\left(\varphi_{1}\left(C_{\lambda}, X\right)\right)^{M}$ e $\left(\varphi_{2}\left(C_{\lambda}, X\right)\right)^{M} ;\left(\varphi_{1}\left(C_{\lambda}, X\right)\right)^{M}$ é a expressão

$$
\forall x \in X \cap M\left(\exists V \in C_{\lambda} \cap M(x \in V)\right),
$$

o que implica que

$$
X \cap M \subset \bigcup\left(C_{\lambda} \cap M\right)
$$

assim,

$$
\mathfrak{C}_{\lambda}=\left\{V \cap M: V \in C_{\lambda} \cap M\right\}
$$

é uma cobertura aberta de $X_{M}$.

Agora, $\left(\varphi_{2}\left(C_{\lambda}, X\right)\right)^{M}$ é a expressão

$$
\forall E \in M\left(\left(E \in\left[C_{\lambda}\right]^{<\left|C_{\lambda}\right|}\right)^{M} \Rightarrow \exists x \in X \cap M(x \notin \cup E)^{M}\right)
$$

que por elementariedade é equivalente a

$$
\forall E \in M\left(E \in\left[C_{\lambda}\right]^{<\left|C_{\lambda}\right|} \Rightarrow \exists x \in X \cap M(x \notin \cup E)\right)
$$

Vamos mostrar que $\mathfrak{C}_{\lambda}$ não tem subcobertura de cardinalidade $\leq \lambda$. Dado qualquer $D \in\left[C_{\lambda} \cap M\right]^{\leq \lambda}$, existe algum $E \in Q_{\lambda}$ tal que $D \subset E$. Como $E \in$ $M \cap\left[C_{\lambda}\right]^{<\left|C_{\lambda}\right|}$ e vale $\left(\varphi_{2}\left(C_{\lambda}, X\right)\right)^{M}$, temos que

$$
\mathfrak{D}=\{V \cap M: V \in D\}
$$

não é subcobertura de $\mathfrak{C}_{\lambda}$. 
Teorema 4.3.13 L reflete todos os cardinais fortemente inacessiveis.

Demonstração. Sejam $\kappa$ um cardinal fortemente inacessível e $X$ um espaço topológico. Considerando o Teorema 4.3.4, só há algo a se provar se $L(X)=\kappa \mathrm{e}$ $\kappa \notin l S(X)$. Então, dado qualquer $\lambda<\kappa$, existe algum $\mu \in l S(X)$, com $\lambda<\mu<\kappa$. Temos então que

$$
\mu^{\lambda} \leq \mu^{\mu}=2^{\mu}<\kappa
$$

$\log 0$

$$
\operatorname{cov}\left(\mu, \mu, \lambda^{+}\right) \leq \operatorname{cov}\left(\mu, \lambda^{+}, \lambda^{+}\right)=\operatorname{cf}\left([\mu]^{\lambda}, \subset\right) \leq \mu^{\lambda}<\kappa,
$$

e podemos aplicar o Teorema 4.3.12.

Com isto, podemos reformular os três primeiros itens do Teorema 4.1 .1 como a seguir:

\section{Teorema 4.3.14}

1. L reflete todo cardinal sucessor e todo cardinal fortemente inacessivel;

2. L reflete todo cardinal limite forte para a classe dos espaços $T_{2}$;

3. $(G C H) L$ reflete todos os cardinais para a classe dos espaços $T_{2}$.

\subsection{Reflexão em cardinais fracamente inacessíveis}

Nesta seção, examinaremos a seguinte questão:

Problema 4.4.1 Podemos demonstrar em ZFC que $L$ reflete todos os cardinais fracamente inacessiveis?

De acordo com o que foi feito na seção anterior, podemos é claro, provar esse resultado de reflexão em $\mathrm{ZFC}+\mathrm{GCH}$.

Nesta seção, daremos algumas respostas parciais a esta pergunta, e descreveremos algumas características necessárias a um eventual contra-exemplo (consistente) para o Problema 4.4.1. Vejamos o seguinte resultado: 
Teorema 4.4.2 L reflete todos os cardinais fracamente inacessiveis para a classe

$$
\{X: l l(X)=L(X)\}
$$

Demonstração. Seja $\kappa$ um cardinal fracamente inacessível. Vamos supor que $L(X)=\kappa$ e $\kappa \notin l S(X)$, caso contrário o resultado seria imediato pelo Teorema 4.3.4. Para cada cardinal $\lambda<\kappa$, como $l l(X)=\kappa$, então existe uma cobertura aberta $\widetilde{C_{\lambda}}$ de $X$ tal que $c f(|S|)>\lambda$ para toda subcobertura $S$ de $\widetilde{C_{\lambda}}$. Defina $\mu=m\left(\widetilde{C_{\lambda}}\right)$, e seja $C_{\lambda}$ uma subcobertura de $\widetilde{C_{\lambda}}$ tal que $\left|C_{\lambda}\right|=\mu$. É fácil ver que $\mu \in l S(X) \mathrm{e}$

$$
\lambda<c f(\mu) \leq \mu<\kappa .
$$

Para obter o resultado, basta verificar então que

$$
\operatorname{cov}\left(\mu, \mu, \lambda^{+}\right) \leq \kappa
$$

e aplicar o Teorema 4.3.12. De fato, neste caso teremos pela Proposição 4.3.11 que $\operatorname{cov}\left(\mu, \mu, \lambda^{+}\right) \leq \operatorname{cf}(\mu)$.

De acordo com o teorema acima, em um eventual contra-exemplo deveria ocorrer $l l(X)<L(X)=\kappa$, onde $\kappa$ é fracamente inacessível. Os exemplos que conhecemos da literatura de espaços $X \operatorname{com} l l(X)<L(X)$ são os (poucos) exemplos de espaços linearmente Lindelöf mas não Lindelöf, ou seja, espaços $X$ com $\omega=l l(X)<L(X)$. Entre os exemplos mais conhecidos, figuram o de Miščenko, o de Gruenhage-Buzyakova, e os exemplos localmente compactos de Kunen (ver [39] e [18]). Se $X$ for qualquer um desses exemplos, temos $L(X)=\aleph_{\omega}$ ou $L(X)=\beth_{\omega}$, portanto $c f(L(X))=\omega=l l(X)$. Não conhecemos nenhum exemplo de espaço onde $c f(L(X))>l l(X)$, o que motiva a seguinte questão:

Problema 4.4.3 Podemos demonstrar que para todo espaço topológico $X$,

$$
c f(L(X)) \leq l l(X) ?
$$

Uma resposta afirmativa a esta pergunta, responderia de forma afirmativa também o Problema 4.4.1.

Vejamos agora mais uma condição topológica que também irá implicar a reflexão de $L$ nos cardinais fracamente inacessíveis. Primeiro, não é difícil notar que, para todo espaço topológico $X$, o extent é limitado pelo grau de Lindelöf linear, ou seja, $e(X) \leq l l(X)$. Iremos agora definir a seguinte função cardinal: 
Definição 4.4.4 Dado um espaço topológico $X, m l(X)$ será o menor cardinal infinito $\lambda$ que satisfaça a seguinte condição: toda cobertura aberta $C$ tem algum refinamento aberto $R$ tal que, para todo $x \in X,|\{U \in R: x \in U\}| \leq \lambda$.

Com isso, os espaços metaLindelöf são exatamente os espaços onde a função cardinal $m l$ vale $\omega$. O próximo resultado é uma generalização do Teorema 3.1.4 de $[15]$ :

Teorema 4.4.5 Seja $X$ um espaço topológico $T_{1}$. Então, $L(X)=e(X) \cdot m l(X)$.

Demonstração. A desigualdade $e(X) \cdot m l(X) \leq L(X)$ é evidente. Vamos agora supor que $\lambda=e(X) \cdot m l(X)$ e $L(X)>\lambda$. Sejam $\mu$ o menor cardinal infinito tal que $\mu \in l S(X)$ e $\mu>\lambda$, e $C$ uma cobertura aberta de $X$ tal que $|C|=m(C)=\mu$. Como $m l(X) \leq \lambda, C$ possui um refinamento aberto $R$ tal que, para todo $x \in X$, $\left|\mathcal{W}_{x}\right| \leq \lambda$, onde $\mathcal{W}_{x}=\{U \in R: x \in U\}$. Como $R$ refina $C, m(R) \geq m(C)=\mu$.

Vamos agora construir a seguinte sequência $\left\{x_{\alpha}: \alpha<\mu\right\}$ em $X$ : supondo já escolhidos $x_{\beta}$ para todo $\beta<\alpha$, temos que

$$
\left|\bigcup_{\beta<\alpha} \mathcal{W}_{x_{\beta}}\right|<\mu
$$

logo podemos escolher algum

$$
x_{\alpha} \in X \backslash \bigcup_{\beta<\alpha}\left(\cup \mathcal{W}_{x_{\beta}}\right) .
$$

Agora, $Y=\left\{x_{\alpha}: \alpha<\mu\right\}$ é um subespaço discreto de $X$, pois dados $\alpha<\beta<\mu$, $\mathcal{W}_{x_{\alpha}} \cap \mathcal{W}_{x_{\beta}}=\emptyset$. Além disso $Y$ é fechado em $X$. De fato, se existisse $x \in \bar{Y} \backslash Y$, teríamos um $V \in R$ tal que $x \in V$. Como $X$ é $T_{1}$, existiriam $\alpha<\beta<\mu$ tais que $x_{\alpha}, x_{\beta} \in V, \log V \in \mathcal{W}_{x_{\alpha}} \cap \mathcal{W}_{x_{\beta}}$, um absurdo.

Com isso, teríamos que

$$
e(X) \geq|Y|=\mu>\lambda
$$

contradição.

Corolário 4.4.6 Seja $X$ um espaço topológico $T_{1}$. Então, $L(X)=l l(X) \cdot m l(X)$.

Corolário 4.4.7 Seja $\kappa$ um cardinal fracamente inacessivel. L reflete $\kappa$ para a classe dos espaços topológicos $\left\{X: X\right.$ é $T_{1}$ e $\left.m l(X)<\kappa\right\}$. 
Demonstração. Se $L(X) \neq \kappa$, temos o resultado pelo Teorema 4.3.4. Caso contrário, de acordo com o corolário anterior, devemos ter $l l(X)=\kappa$, e então podemos aplicar o Teorema 4.4.2.

Agora usando Teoria PCF, podemos encontrar mais condições suficientes para que $L$ reflita todo cardinal fracamente inacessível. O resultado desta teoria que usaremos é o Teorema 7.2 de [1]:

Teorema 4.4.8 Se $\aleph_{\delta}$ é um cardinal singular $\operatorname{com} \delta<\aleph_{\delta}$, então

$$
c f\left(\left[\aleph_{\delta}\right]^{|\delta|}, \subset\right)<\aleph_{|\delta|^{++++}} .
$$

Quando $\delta=\omega$, temos o conhecido resultado $c f\left(\left[\aleph_{\omega}\right]^{\omega}, \subset\right)<\aleph_{\omega_{4}}$. Com o teorema acima, podemos mostrar o seguinte resultado.

Teorema 4.4.9 L reflete todos os cardinais fracamente inacessiveis para a classe

$$
\{X: L(X)=\sup (l S(X) \backslash F I X)\} .
$$

Demonstração. Considerando os Teoremas 4.3 .4 e 4.4.2, só há algo a se provar se $L(X)=\kappa, \kappa \notin l S(X)$ e $l l(X)<\kappa$. Para cada cardinal $\lambda<\kappa$, existe algum $\mu \in l S(X) \backslash F I X$ tal que

$$
\mu>\aleph_{\lambda+l l(X)}
$$

Como $\mu \in l S(X)$ não é ponto fixo e $\kappa \notin l S(X), \mu=\aleph_{\delta}$ para algum ordinal $\delta$, com

$$
\lambda<\delta<\mu<\kappa,
$$

$\operatorname{logo} \lambda \leq|\delta|$. Escolha uma cobertura aberta $C_{\lambda}$ de $X$ tal que

$$
\left|C_{\lambda}\right|=m\left(C_{\lambda}\right)=\mu \text {. }
$$

Note que $\mu$ é singular, pois de acordo com o Corolário 1.2.34,

$$
c f(\mu)=c f\left(m\left(C_{\lambda}\right)\right) \leq l l(X)<\mu .
$$

Então, de acordo com o Teorema 4.4.8,

$$
c f\left([\mu]^{|\delta|}, \subset\right)<\aleph_{|\delta|^{++++}}<\kappa ;
$$


$\log \mathrm{O}$

$$
\operatorname{cov}\left(\mu, \mu, \lambda^{+}\right) \leq \operatorname{cov}\left(\mu, \mu,|\delta|^{+}\right) \leq \operatorname{cov}\left(\mu,|\delta|^{+},|\delta|^{+}\right)=\operatorname{cf}\left([\mu]^{|\delta|}, \subset\right)<\kappa,
$$

e temos o resultado pelo Teorema 4.3.12.

Supondo que existam um espaço $X$ e um cardinal fracamente inacessível $\kappa$, tais que $X$ seja um contra-exemplo consistente para o Problema 4.4.1, além de termos $l l(X)<L(X)=\kappa$, o espectro $l S(X)$ deveria ser composto, a partir de algum cardinal $\lambda<\kappa$, apenas de pontos fixos singulares, ou seja, cardinais $\vartheta$ tais que $c f(\vartheta)<\vartheta=\aleph_{\vartheta}$. Além disso, precisaríamos ter, para cada $\vartheta>\lambda$ em $l S(X)$, $\operatorname{cov}\left(\vartheta, \vartheta, \lambda^{+}\right)>\kappa$.

A dificuldade de encontrar um contra-exemplo satisfazendo as condições acima, especialmente a última, reside em parte no fato de que a aritmética cardinal envolvendo pontos fixos é um terreno ainda em parte inexplorado. Por exemplo, a Teoria PCF é capaz de fornecer limitantes superiores para $2^{\lambda}$ sempre que $\lambda$ seja um cardinal singular e limite forte, mas não ponto fixo (Teorema 1.1.15). Quando $\lambda$ é um ponto fixo, em alguns casos há resultados fornecendo limitantes para $2^{\lambda}$. Por exemplo, se $\kappa$ for o primeiro cardinal fracamente inacessível, e $\lambda<\kappa$ qualquer cardinal singular e limite forte, com $c f(\lambda)>\omega$, então $2^{\lambda}<\kappa$, sendo $\lambda$ ponto fixo ou não (ver [33]). Resultados semelhantes foram obtidos para alguns pontos fixos de cofinalidade enumerável (ver [17]). No entanto, para o primeiro ponto fixo

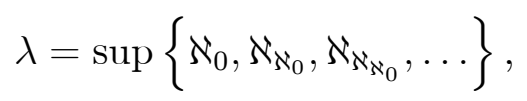

que tem cofinalidade enumerável, Moti Gitik mostrou recentemente em [17] que não há limitantes para $2^{\lambda}$, respondendo uma questão de Shelah em [41]. Mais precisamente, dado qualquer cardinal $\mu>\lambda$, é consistente (supondo-se a existência de determinados grandes cardinais) que $\mathrm{GCH}$ valha para todos os cardinais menores que $\lambda$, mas que $2^{\lambda} \geq \mu$.

Em [33] é citada a seguinte frase de Saharon Shelah a respeito desse tópico, extraída da página 358 de [41]: ${ }^{1}$

... before 1980 we could have thought that for example for strong limit $\lambda$ of cofinality $\aleph_{0}$ there are no restrictions on $2^{\lambda}$... we know now there are some but have less clear idea what to expect for fix points.

\footnotetext{
${ }^{1}$ Foi em 1980 que Shelah mostrou que se $\aleph_{\omega}$ for limite forte, então $2^{\aleph_{\omega}}<\aleph_{\left(2^{\aleph_{0}}\right)^{+}}$, dessa forma estendendo o Teorema de Galvin-Hajnal.
} 
Considerando a Proposição 4.3.8 e o resultado de Gitik sobre o primeiro ponto fixo $\lambda$, temos que não se pode provar em ZFC que exista algum cardinal $\mu$ tal que

$$
c f\left([\lambda]^{c f(\lambda)}, \subset\right)=c f\left([\lambda]^{\omega}, \subset\right)=\operatorname{cov}\left(\lambda, \omega_{1}, \omega_{1}\right)<\mu
$$

(a menos que sejam inconsistentes com ZFC os grandes cardinais assumidos em $[17])$.

Dados cardinais $\nu$ e $\eta$, com $\nu<\eta$, temos que $\operatorname{cov}\left(\eta, \eta, \nu^{+}\right) \leq \operatorname{cov}\left(\eta, \nu^{+}, \nu^{+}\right)$, e portanto em príncipio, apesar do resultado de [17], poderiam sempre existir limitantes para $\operatorname{cov}\left(\eta, \eta, \nu^{+}\right)$, que estruturalmente é o tipo de valor combinatório que aparece nas hipóteses do Teorema 4.3.12. Por exemplo, se $\lambda$ for o primeiro ponto fixo, então $\operatorname{cov}\left(\lambda^{+}, \omega_{1}, \omega_{1}\right) \geq \operatorname{cov}\left(\lambda, \omega_{1}, \omega_{1}\right)$, mas $\operatorname{cov}\left(\lambda^{+}, \lambda^{+}, \omega_{1}\right)=\lambda^{+}$, o que ilustra como $\operatorname{cov}\left(\eta, \eta, \nu^{+}\right)$pode ser muito menor que $\operatorname{cov}\left(\eta, \nu^{+}, \nu^{+}\right)$. A relevância do resultado principal de [17] para o problema de reflexão de $L$ se torna mais clara com o próximo resultado, cuja demonstração se encontra no Apêndice A.

Proposição 4.4.10 Se $\lambda$ for o primeiro ponto fixo da função $\aleph$, e $\vartheta$ for qualquer cardinal menor que $\lambda$, então não é possivel provar em ZFC que há um limitante para $\operatorname{cov}\left(\lambda, \lambda, \vartheta^{+}\right)$(supondo a consistência dos grandes cardinais assumidos em $[17])$.

Iremos prosseguir nossa análise do Problema 4.4.1 por meio de uma hipótese consideravelmente mais fraca que GCH: SSH (Shelah's Strong Hypothesis), que possui a seguinte caracterização equivalente mais conveniente para nossos propósitos [37]:

Teorema 4.4.11 SSH é equivalente à seguinte afirmação: dados cardinais $\mu$ e $\lambda$, $\operatorname{com} \mu \geq \lambda=\operatorname{cf}(\lambda) \geq \omega_{1}, \operatorname{cov}(\mu, \lambda, \lambda)=\mu$ se cf $(\mu) \geq \lambda$, e $\operatorname{cov}(\mu, \lambda, \lambda)=\mu^{+}$ caso contrário.

De acordo com [40], tanto GCH quanto "não existe $0^{\sharp " ~ i m p l i c a m ~ S S H ; ~ e ~ d e ~}$ acordo com [16], SSH também é implicado pela hipótese FRP (Fodor-type Reflection Principle). Ainda de acordo com [37], SSH implica SCH (Singular Cardinal Hypothesis), mas é um problema em aberto se a falha de SSH é equiconsistente com a falha de SCH (e esta última é equiconsistente com a existência de um cardinal mensurável $\kappa$ de ordem de Mitchell $\left.\kappa^{++}\right)$. 
$\mathrm{Na}$ literatura não encontramos uma demonstração do próximo teorema, que demonstramos no Apêndice A. Parte do argumento é baseado na demonstração em [23] (Corolário 18.33) de que se $0^{\sharp}$ não existe, então vale $\mathrm{SCH}$.

Teorema 4.4.12 Se $0^{\sharp}$ não existe, então vale $S S H$.

Podemos também caracterizar SSH em função da existência de certos submodelos elementares, o que é relevante para o estudo realizado em [38] (ver demonstração no Apêndice A):

Proposição 4.4.13 SSH equivale à seguinte afirmação: "Dados cardinais infinitos $\lambda$ e $\mu$, com $\mathrm{cf}(\mu)>\lambda$, existe submodelo elementar $\lambda$-covering de cardinalidade $\mu^{\prime \prime}$.

Vamos introduzir agora duas hipóteses ainda mais fracas que SSH.

Definição 4.4.14 $\boldsymbol{w H 1}$ : se $\kappa$ for um cardinal fracamente inacessível $e \lambda$ e $\mu$ forem cardinais com $\lambda<\mu<\kappa$, então $\operatorname{cov}\left(\mu, \mu, \lambda^{+}\right) \leq \kappa$.

Definição 4.4.15 $\boldsymbol{w H 2 :}$ se $\kappa$ for um cardinal fracamente inacessivel e $\lambda$ um cardinal menor que $\kappa$, então existe algum $\mu$, com $\lambda<\mu<\kappa$, tal que $\operatorname{cov}\left(\beta, \beta, \lambda^{+}\right) \leq \kappa$ sempre que $\mu \leq \beta \leq \kappa$.

É imediato que $\mathrm{SSH} \Rightarrow \mathrm{wH} 1 \Rightarrow \mathrm{wH} 2$. A falha de $\mathrm{SSH}$ pode ser obtida com a falha de $\mathrm{SCH}$, mas esta não implica na falha de wH1: considere a extensão genérica de Woodin (ver Teorema 36.6 de [23]) onde $2^{\kappa}=\kappa^{++}$para todo cardinal $\kappa$ (obtida supondo que exista um cardinal supercompacto). Nessa extensão, não vale SCH, pois caso contrário teríamos $2^{\lambda}=\lambda^{+}$para cada cardinal singular $\lambda$. Mas vale $\mathrm{wH}$, pois dados $\lambda, \mu$ e $\kappa$ como enunciados,

$$
\operatorname{cov}\left(\mu, \mu, \lambda^{+}\right) \leq \operatorname{cf}\left([\mu]^{\lambda}, \subset\right) \leq \mu^{\lambda} \leq 2^{\mu}=\mu^{++}<\kappa .
$$

A falha de wH1 pode ser obtida na extensão genérica de [17], de acordo com a Proposição 4.4.10. Os grandes cardinais assumidos no teorema principal de [17] são mais fortes que aqueles necessários para fazer falhar $\mathrm{SCH}$.

Quanto à wH2, não temos uma resposta para a seguinte questão: 
Problema 4.4.16 $Z F C \vdash w H 2$ ?

A relevância da pergunta acima para a reflexão de $L$ se manifesta nos três próximos resultados, que estão entre os mais importantes deste trabalho.

Teorema 4.4.17 (wH2) L reflete todos os cardinais fracamente inacessiveis.

Demonstração. Sejam $X$ um espaço topológico e $\kappa$ um cardinal fracamente inacessível. Se $L(X) \neq \kappa$ ou $\kappa \in l S(X)$, temos o resultado pelo Teorema 4.3.4. Caso contrário, dado qualquer $\lambda<\kappa$, escolha algum $\beta \in l S(X)$ tal que $\lambda<\mu \leq \beta$, onde $\mu$ é dado por wH2. Podemos então aplicar o Teorema 4.3.12, pois $\operatorname{cov}\left(\beta, \beta, \lambda^{+}\right) \leq$ $\kappa$.

Corolário 4.4.18 (SSH) L reflete todos os cardinais regulares.

Corolário 4.4.19 (não existe $0^{\sharp}$ ) L reflete todos os cardinais regulares.

Suponha que exista algum cardinal fracamente inacessível $\kappa$, e um conjunto $F \subset \kappa, \operatorname{com} \sup F=\kappa$, onde para cada $\alpha \in F$ :

- $\alpha$ é um cardinal com $c f(\alpha)=\omega$ e $\aleph_{\alpha}=\alpha$;

- dado qualquer cardinal $\mu$, existe uma extensão genérica onde $\alpha$ é limite forte e $2^{\alpha} \geq \mu$ (como ocorre para o primeiro ponto fixo em [17]).

Este conjunto de condições seria suficiente para falhar $\mathrm{wH}_{2}$, considerando o Corolário A.1.5. Deve-se notar no entanto que o conjunto $F$ acima não poderia ser simplesmente o conjunto de todos os pontos fixos menores que $\kappa$ de cofinalidade enumerável (ver em [17] o exemplo do primeiro ponto fixo de ordem $\omega$ ).

Para concluir, se existir um contra-exemplo consistente para a afirmação " $L$ reflete todos os cardinais regulares", este contra-exemplo precisaria das seguintes condições, de acordo com o que vimos:

- é necessário assumir a negação de wH2 (se isto for consistente com ZFC), e portanto, a existência de $0^{\sharp}$, ou possivelmente algum outro axioma de grandes cardinais ainda mais forte; 
- nessa axiomática de conjuntos, construir um espaço topológico $X$ com as seguintes características, onde $\kappa$ é um cardinal fracamente inacessível:

$$
\begin{aligned}
& -|X|>\kappa \\
& -L(X)=\kappa \\
& -l l(X)<\kappa \text { (o que implica } \kappa \notin l S(X)) \\
& -m l(X)=\kappa \text { ou } X \text { não ser } T_{1} \\
& \text { - existir algum cardinal } \xi<\kappa \text { tal que, para todo } \vartheta \in l S(X) \operatorname{com} \vartheta>\xi, \\
& \quad \aleph_{\vartheta}=\vartheta \text { e } c f(\vartheta)<\vartheta
\end{aligned}
$$

\subsection{Reflexão de ll}

Nesta seção estudaremos a propriedade de reflexão de cardinais regulares, para a função cardinal $l l$. Os métodos usados são semelhantes aos usados para a função cardinal $L$.

Definição 4.5.1 Se $X$ for um espaço topológico, defina

$$
l l S(X)=\{m(C): C \text { é cobertura aberta de } X \text {, bem ordenada pela inclusão }\} .
$$

Considerando a equivalência entre os itens (a) e (b) da Proposição 1.2.33, é fácil ver que $l l(X)=\sup l l S(X)$.

Dada uma cobertura aberta $C$ de $X$, bem ordenada pela inclusão, e uma subcobertura $S$ de tipo de ordem mínimo, teremos que $m(C)=|S|, \operatorname{logo} m(C)$ é um cardinal regular. Portanto, diferentemente do que ocorre com $l S(X), l l S(X)$ é composto exclusivamente de cardinais regulares. Na verdade, é fácil mostrar que

$$
l l S(X)=\{\lambda \in l S(X): c f(\lambda)=\lambda\} .
$$

Teorema 4.5.2 A função cardinal ll reflete todos os cardinais regulares.

Demonstração. A demonstração é inteiramente análoga ao do Teorema 4.3.12, com as seguintes considerações: 
- dado qualquer $\lambda<\kappa$, existe $\mu \in l l S(X) \operatorname{com} \lambda<\mu \leq \kappa$ e $\operatorname{cov}\left(\mu, \mu, \lambda^{+}\right) \leq$ $\mu \leq \kappa$, pois $l l S(X)$ contém apenas cardinais regulares;

- $l l(X \cap M) \geq l l\left(X_{M}\right)$, à semelhança do que ocorre com $L$;

- se $C_{\lambda}$ for bem ordenado pela inclusão, $\mathfrak{C}_{\lambda}$ também será. 


\section{Apêndice A}

\section{Resultados combinatórios}

Neste apêndice enunciamos e demonstramos resultados sobre cov que são necessários ao capítulo 4, mas que não constituem o objetivo principal do trabalho, que é o de estudar reflexão de funções cardinais.

\section{A.1 Propriedades gerais}

Proposição A.1.1 Sejam $\mu, \chi, \lambda, \mu^{\prime}, \chi^{\prime}, \lambda^{\prime}$ cardinais infinitos, com $\mu^{\prime} \geq \mu \geq \chi^{\prime} \geq$ $\chi \geq \lambda^{\prime} \geq \lambda$. Temos então:

$$
\begin{aligned}
& \text { 1. } \operatorname{cov}(\mu, \chi, \lambda) \leq \operatorname{cov}\left(\mu^{\prime}, \chi, \lambda\right) \text {; } \\
& \text { 2. } \operatorname{cov}(\mu, \chi, \lambda) \geq \operatorname{cov}\left(\mu, \chi^{\prime}, \lambda\right) \text {; } \\
& \text { 3. } \operatorname{cov}(\mu, \chi, \lambda) \leq \operatorname{cov}\left(\mu, \chi, \lambda^{\prime}\right) \text {. }
\end{aligned}
$$

Demonstração. Vamos demonstrar aqui a propriedade (1), as propriedades (2) e (3) são imediatas.

Seja uma família $F^{\prime} \subset\left[\mu^{\prime}\right]^{<\chi}, \operatorname{com}\left|F^{\prime}\right|=\operatorname{cov}\left(\mu^{\prime}, \chi, \lambda\right)$, tal que, para todo $a \in\left[\mu^{\prime}\right]^{<\lambda}$ existe algum $b \in F^{\prime}$ tal que $a \subset b$. Defina então $F=\left\{x \cap \mu: x \in F^{\prime}\right\}$. Teremos que $F \subset[\mu]^{<\chi},|F| \leq \operatorname{cov}\left(\mu^{\prime}, \chi, \lambda\right)$, e para todo $a \in[\mu]^{<\lambda}$ existe algum $b \in F$ tal que $a \subset b$. Portanto, $\operatorname{cov}(\mu, \chi, \lambda) \leq|F|$.

Proposição A.1.2 Sejam $\mu, \chi, \lambda$ cardinais infinitos, com $\mu \geq \chi \geq \lambda$. Se $\mu$ for regular ou $\mu>\chi$, então $\operatorname{cov}(\mu, \chi, \lambda) \geq \mu$. 
Demonstração. Se $\mu$ for regular, então $\operatorname{cov}(\mu, \chi, \lambda) \geq \mu$ pois caso contrário $\mu$ seria uma união de menos do que $\mu$ subconjuntos de cardinalidade $<\chi$.

Se $\mu$ for singular e maior que $\chi$, para cada cardinal $\nu \in[\chi, \mu)$,

$$
\operatorname{cov}(\mu, \chi, \lambda) \geq \operatorname{cov}\left(\nu^{+}, \chi, \lambda\right) \geq \nu^{+} .
$$

Portanto, $\operatorname{cov}(\mu, \chi, \lambda) \geq \mu$.

Proposição A.1.3 Sejam $\mu$ e $\lambda$ cardinais infinitos, com $\lambda \leq c f(\mu)$. Então $\operatorname{cov}(\mu, \mu, \lambda) \leq c f(\mu)$.

Demonstração. Como $\lambda \leq c f(\mu)$, todo elemento de $[\mu]^{<\lambda}$ é limitado em $\mu$. Desta forma, tomando uma sequência crescente de ordinais $\left(\gamma_{\alpha}: \alpha<c f(\mu)\right)$ cofinal em $\mu$, a família $\left\{\gamma_{\alpha}: \alpha<c f(\mu)\right\}$ testemunhará que $\operatorname{cov}(\mu, \mu, \lambda) \leq c f(\mu)$.

A demonstração da próxima proposição segue um argumento sugerido em [36].

Proposição A.1.4 Dados cardinais $\eta$, $\nu$ e $v$, com $\eta \geq \nu \geq v$,

$$
\operatorname{cov}(\eta, \nu, v)=\sum_{\beta \in[\nu, \eta]} \operatorname{cov}(\beta, \beta, v) .
$$

Demonstração. Primeiramente, só há algo a se demonstrar, se $\eta>\nu$.

Defina

$$
\zeta=\sum_{\beta \in[\nu, \eta]} \operatorname{cov}(\beta, \beta, v) .
$$

Temos pela Proposição A.1.2 que

$$
\operatorname{cov}(\eta, \nu, v) \geq \eta \geq|[\nu, \eta]| \text {. }
$$

Dado qualquer $\beta \in[\nu, \eta]$,

$$
\operatorname{cov}(\eta, \nu, v) \geq \operatorname{cov}(\beta, \beta, v)
$$

$\log 0 \operatorname{cov}(\eta, \nu, v) \geq \zeta$.

Para mostrar que $\operatorname{cov}(\eta, \nu, v) \leq \zeta$, vamos construir uma árvore $(T, \leq)$ como a seguir:

- cada elemento de $T$ será um $S \subset \eta$;

- T terá uma raiz, que será $\eta$;

- as folhas de $T$ serão exatamente os elementos $S \operatorname{com}|S|<\nu$; 
- para cada elemento $S$ de $T$ com $|S| \geq \nu$, os sucessores de $S$ serão os elementos de uma família $F_{S} \subset[S]^{<|S|}$, com $\left|F_{S}\right|=\operatorname{cov}(|S|,|S|, v)$, tal que para todo $a \in[S]^{<v}$, existe algum $b \in F_{S}$ tal que $a \subset b$.

Para cada elemento que não seja uma folha, a quantidade de sucessores é $\operatorname{cov}(|S|,|S|, v) \leq \zeta$. Cada ramo da árvore é finito, pois está associado a uma sequência estritamente decrescente de cardinais. Portanto, se $\xi$ for a quantidade de ramos da árvore, que nesse caso é também a quantidade de folhas, então $\xi \leq \zeta$.

Por fim, mostraremos que $\operatorname{cov}(\eta, \nu, v) \leq \xi$, exibindo como obter uma família $F \subset[\eta]^{<\nu}, \operatorname{com}|F| \leq \xi$, tal que para todo $a \in[\eta]^{<v}$, existe algum $b \in F$ tal que $a \subset b$. A família $F$ será o conjunto de todas as folhas de $T$, pois dado qualquer $a \in[\eta]^{<v}$, basta escolher um ramo da árvore tal que $a$ esteja contido em todos os elementos desse ramo; $b \in F$ será então a folha desse ramo.

Corolário A.1.5 Se $\lambda$ for um cardinal singular e limite forte, então

$$
2^{\lambda}=\operatorname{cov}\left(\lambda, \lambda,(c f(\lambda))^{+}\right) .
$$

Demonstração. Pela Proposição 4.3.8,

$$
2^{\lambda}=c f\left([\lambda]^{c f(\lambda)}, \subset\right)=\operatorname{cov}\left(\lambda,(c f(\lambda))^{+},(c f(\lambda))^{+}\right) .
$$

Pela proposição anterior, teremos que:

$$
\begin{gathered}
\operatorname{cov}\left(\lambda,(c f(\lambda))^{+},(c f(\lambda))^{+}\right)=\sum_{\beta \in\left[(c f(\lambda))^{+}, \lambda\right]} \operatorname{cov}\left(\beta, \beta,(c f(\lambda))^{+}\right)= \\
\operatorname{cov}\left(\lambda, \lambda,(c f(\lambda))^{+}\right)+\sum_{\beta \in\left[(c f(\lambda))^{+}, \lambda[\right.} \operatorname{cov}\left(\beta, \beta,(c f(\lambda))^{+}\right) .
\end{gathered}
$$

Para cada $\beta \in\left[(c f(\lambda))^{+}, \lambda[\right.$,

$$
\operatorname{cov}\left(\beta, \beta,(c f(\lambda))^{+}\right) \leq \operatorname{cf}\left([\beta]^{c f(\lambda)}, \subset\right) \leq \beta^{c f(\lambda)}<\lambda,
$$

pelo fato de $\lambda$ ser limite forte. Com isso,

$$
\sum_{\beta \in\left[(c f(\lambda))^{+}, \lambda[\right.} \operatorname{cov}\left(\beta, \beta,(c f(\lambda))^{+}\right) \leq \lambda,
$$

$\log \mathrm{O}$

$$
2^{\lambda} \leq \lambda+\operatorname{cov}\left(\lambda, \lambda,(c f(\lambda))^{+}\right)
$$

o que implica que

$$
2^{\lambda}=\operatorname{cov}\left(\lambda, \lambda,(c f(\lambda))^{+}\right) .
$$


Corolário A.1.6 Se $\lambda$ for o primeiro ponto fixo da função $\aleph$, e $\vartheta$ for qualquer cardinal menor que $\lambda$, então não é possivel provar em ZFC que há um limitante para $\operatorname{cov}\left(\lambda, \lambda, \vartheta^{+}\right)$(supondo a consistência dos grandes cardinais assumidos em $[17])$.

Demonstração. No resultado principal de [17], dado qualquer cardinal $\mu, \lambda$ é limite forte e

$$
\operatorname{cov}\left(\lambda, \lambda, \vartheta^{+}\right) \geq \operatorname{cov}\left(\lambda, \lambda, \omega_{1}\right)=\operatorname{cov}\left(\lambda, \lambda,(\operatorname{cf}(\lambda))^{+}\right)=2^{\lambda} \geq \mu .
$$

\section{A.2 Propriedades de SSH}

Para o próximo resultado, vamos precisar da seguinte definição [37]:

Definição A.2.1 Dados cardinais $\mu, \chi, \lambda e \sigma$, com $\mu \geq \chi \geq \lambda \geq \omega e \lambda \geq \sigma \geq 2$, $\operatorname{cov}(\mu, \chi, \lambda, \sigma)$ é a menor cardinalidade de algum $X \subset[\mu]^{<\chi}$ tal que, para qualquer $a \in[\mu]^{<\lambda}$, existe um $Q \in[X]^{<\sigma}$ com $a \subset \cup Q$.

É fácil ver que

$$
\operatorname{cov}(\mu, \chi, \lambda)=\operatorname{cov}(\mu, \chi, \lambda, 2)
$$

e que

$$
\operatorname{cov}(\mu, \chi, \lambda, \sigma) \geq \operatorname{cov}\left(\mu, \chi, \lambda, \sigma^{\prime}\right)
$$

quando $\sigma \leq \sigma^{\prime}$.

Teorema A.2.2 Se $0^{\sharp}$ não existe, então vale $S S H$.

Demonstração. Sejam cardinais $\mu$ e $\lambda$ tais que $\mu \geq \lambda=c f(\lambda) \geq \omega_{1}$.

Primeiramente, $\operatorname{cov}(\mu, \lambda, \lambda) \geq \mu$ de acordo com a Proposição A.1.2, e se $c f(\mu)<\lambda$,

$$
\operatorname{cov}(\mu, \lambda, \lambda) \geq \operatorname{cov}\left(\mu, \mu,(\operatorname{cf}(\mu))^{+}\right) \geq \operatorname{cov}\left(\mu, \mu,(\operatorname{cf}(\mu))^{+}, \operatorname{cf}(\mu)\right) \geq \mu^{+}
$$

(Fato 1 em [36]).

Se $\lambda \geq \omega_{2}, \operatorname{cov}(\mu, \lambda, \lambda) \leq \mu^{+}$, pois de acordo com o Teorema 18.30 de [23] (Jensen's Covering Theorem) todo $a \in[\mu]^{<\lambda}$ está contido em algum conjunto construtível $b \in[\mu]^{\omega_{1}+|a|}$, e $\left|\mathcal{P}^{L}(\mu)\right| \leq \mu^{+}$. 
Com isso, e usando o item (1) do Lema 5.10 de [1], $\operatorname{cov}\left(\mu, \omega_{1}, \omega_{1}\right)=\operatorname{cf}\left([\mu]^{\omega}, \subset\right) \leq c f\left([\mu]^{\omega_{1}}, \subset\right) \cdot \operatorname{cf}\left(\left[\omega_{1}\right]^{\omega}, \subset\right) \leq \mu^{+} \cdot \omega_{1}=\mu^{+}$.

Finalmente, se $c f(\mu) \geq \lambda$, pela Proposição A.1.4,

$$
\begin{gathered}
\operatorname{cov}(\mu, \lambda, \lambda)=\sum_{\beta \in[\lambda, \mu]} \operatorname{cov}(\beta, \beta, \lambda)=\operatorname{cov}(\mu, \mu, \lambda)+\sum_{\beta \in[\lambda, \mu[} \operatorname{cov}(\beta, \beta, \lambda) \\
\leq \operatorname{cf}(\mu)+\sum_{\beta \in[\lambda, \mu[} \operatorname{cov}(\beta, \lambda, \lambda) \leq \mu+\sum_{\beta \in[\lambda, \mu[} \beta^{+}=\mu .
\end{gathered}
$$

Proposição A.2.3 SSH equivale à seguinte afirmação: "Dados cardinais infinitos $\lambda$ e $\mu$, com $c f(\mu)>\lambda$, existe submodelo elementar $\lambda$-covering de cardinalidade $\mu "$.

Demonstração. Primeiro vamos provar a afirmação a partir de $\mathrm{SSH}$. Como $c f(\mu)>$ $\lambda$, então

$$
c f\left([\mu]^{\lambda}, \subset\right)=\operatorname{cov}\left(\mu, \lambda^{+}, \lambda^{+}\right)=\mu .
$$

Podemos então proceder como na demonstração do Teorema 3.1.26 de [38], construindo uma sequência crescente $\left(M_{\alpha}: \alpha<\lambda^{+}\right)$de submodelos elementares, com $\left|M_{\alpha}\right| \leq \mu$ para todo $\alpha<\lambda^{+}$.

Agora suponha que vale a afirmação. Considerando a demonstração do Teorema A.2.2, basta mostrarmos que, se $\mu \geq \lambda=\operatorname{cf}(\lambda) \geq \omega_{1}, \operatorname{cov}(\mu, \lambda, \lambda) \leq \mu$ se $c f(\mu) \geq \lambda$, e $\operatorname{cov}(\mu, \lambda, \lambda) \leq \mu^{+}$se $c f(\mu)<\lambda$.

Se $c f(\mu) \geq \lambda$, dado qualquer $\eta<\lambda$, temos que $c f(\mu)>\eta$, então existe um submodelo elementar $\eta$-covering de cardinalidade $\mu$, logo $\operatorname{cov}\left(\mu, \eta^{+}, \eta^{+}\right) \leq \mu$. Para cada $\eta<\lambda$, seja $C_{\eta} \subset[\mu]^{\eta}$ a família que testemunhe que $\operatorname{cov}\left(\mu, \eta^{+}, \eta^{+}\right) \leq \mu$; então $C=\bigcup_{\eta<\lambda} C_{\eta}$ irá testemunhar que $\operatorname{cov}(\mu, \lambda, \lambda) \leq \mu$.

Finalmente, dados quaisquer $\mu$ e $\lambda \operatorname{com} \mu \geq \lambda=c f(\lambda) \geq \omega_{1}$,

$$
\operatorname{cov}(\mu, \lambda, \lambda) \leq \operatorname{cov}\left(\mu^{+}, \lambda, \lambda\right) \leq \mu^{+},
$$

de acordo com o parágrafo anterior. 


\section{Referências Bibliográficas}

[1] U. Abraham e M. Magidor, Cardinal Arithmetic, Handbook of Set Theory, Springer, 2010, 1149-1227.

[2] O. T. Alas, V. V. Tkachuk e R. G. Wilson, Closures of discrete sets often reflect global properties, Topology Proceedings 25 (2000), 27-44.

[3] A. V. Arhangel'skiı̌ e R. Z. Buzyakova, Convergence in compacta and linear Lindelöfness, Commentationes Mathematicae Universitatis Carolinae 39 (1998), 159-166.

[4] A. V. Arhangel'skiı̌ e R. Z. Buzyakova, On linearly Lindelöf and strongly discretely Lindelöf spaces, Proceedings of the American Mathematical Society 127 (1999), 2449-2458.

[5] L. F. Aurichi, Sobre a hipótese do contínuo algumas aplicações e equivalências, Dissertação de Mestrado. IME-USP, 2005.

[6] R. Z. Buzyakova, Cardinalities of some Lindelöf and $\omega_{1}$-Lindelöf $T_{1} / T_{2}$ spaces, Topology and its Applications 143 (2004), 209-216.

[7] F. Casarrubias-Segura e A. Ramírez-Páramo, Reflection theorems for some cardinal functions, Topology Proceedings 31 (2007), 51-65.

[8] S. Christodoulou, Espaços inicialmente $\kappa$-compactos, Tese de Doutorado. IME-USP, 1994.

[9] R. R. Dias, Reflexão de funções cardinais e da metrizabilidade, Dissertação de Mestrado. IME-USP, 2008.

[10] A. Dow, An introduction to applications of elementary submodels to topology, Topology Proceedings 13 (1988), 17-72.

[11] A. Dow, An empty class of nonmetric spaces, Proceedings of the American Mathematical Society 104 (1988) 999-1001. 
[12] F. W. Eckertson, Images of not Lindelöf spaces and their squares, Topology and its Applications 62 (1995), 255-261.

[13] B. A. Efimov, Mappings and imbeddings of dyadic spaces, Math USSR Sbornik 32 (1977), 45-57.

[14] R. Engelking, General topology, Heldermann, Berlin, 1989.

[15] R. A. Figueiredo, Espaços linearmente Lindelöf, Dissertação de Mestrado. IME-USP, 2010.

[16] S. Fuchino, A. Rinot, Openly generated Boolean algebras and the Fodor-type reflection principle, Fundamenta Mathematicae 212 (2011), 261-283.

[17] M. Gitik, No bound for the first fixed point, Journal of Mathematical Logic 5 (2005), 193-246.

[18] I. Gorelic e I. Juhász, AB-compacta, Commentationes Mathematicae Universitatis Carolinae 49 (2008), 141-146.

[19] A. Hajnal e I. Juhász, Some remarks on a property of topological cardinal functions, Acta Mathematica Academiae Scientiarum Hungaricae 20 (1969), $25-37$.

[20] A. Hajnal e I. Juhász, Having a small weight is determined by the small subspaces, Proceedings of the American Mathematical Society 79 (1980) 657658.

[21] R. E. Hodel, Cardinal functions I, Handbook of set-theoretic topology, NorthHolland, Amsterdam, 1984, 1-61.

[22] R. E. Hodel e J. E. Vaughan, Reflection theorems for cardinal functions, Topology and its Applications 100 (2000), 47-66.

[23] Thomas Jech. Set theory. Springer Monographs in Mathematics. SpringerVerlag, Berlin, 2003. The third millennium edition, revised and expanded.

[24] I. Juhász, Cardinal functions in topology - ten years later, Mathematical Centre Tracts $n^{o} 123$, Amsterdam, 1980.

[25] I. Juhász e Z. Szentmiklóssy, Two results concerning cardinal functions on compact spaces, Proceedings of the American Mathematical Society 90 (1984), 608-610.

[26] I. Juhász, Cardinal functions and reflection, Topology Atlas Preprint $\mathrm{n}^{\circ} 445$, 2000 . 
[27] I. Juhász e Z. Szentmiklóssy, On d-separability of powers and $C_{p}(X)$, Topology and its Applications 155 (2008), 277-281.

[28] I. Juhász, P. Koszmider e L. Soukup, A first countable, initially $\omega_{1}$-compact but non-compact space, Topology and its Applications 156 (2009), 1863-1879.

[29] I. Juhász e W. A. R. Weiss, On the convergence and character spectra of compact spaces, Fundamenta Mathematicae 207 (2010), 179-196.

[30] L. R. Junqueira e F. D. Tall, The topology of elementary submodels, Topology and its Applications 82 (1998), 239-266.

[31] L. R. Junqueira, Upwards preservation by elementary submodels, Topology Proceedings 25 (2000), 225-249.

[32] A. Kanamori, The Higher Infinite. Large Cardinals in Set Theory from Their Beginnings. Springer Monographs in Mathematics. Springer, Berlin, 2003. Second edition.

[33] M. Kojman, Singular Cardinals: from Hausdorff's gaps to Shelah's PCF Theory, Sets and Extensions in the Twentieth Century, volume 6 of Handbook of the History of Logic, Elsevier, 2011, 509-558.

[34] K. Kunen. Set theory. An introduction to independence proofs, volume 102 of Studies in Logic and the Foundations of Mathematics. North-Holland Publishing Co., Amsterdam, 1980.

[35] K. Kunen, Locally compact linearly Lindelöf spaces, Commentationes Mathematicae Universitatis Carolinae 43 (2002), 155-158.

[36] A. Liu, Bounds for Covering Numbers, The Journal of Symbolic Logic 71 (2006), 1303-1310.

[37] P. Matet, Large cardinals and covering numbers, Fundamenta Mathematicae 205 (2009), 45-75.

[38] M. D. Passos, Extensões de submodelos elementares por forcing, Tese de Doutorado. IME-USP, 2007.

[39] E. Pearl, Linearly Lindelöf problems, Open problems in topology II, Elsevier Science, 2007.

[40] S. Shelah, Cardinal Arithmetic for skeptics, Bulletin of the American Mathematical Society 26 (1992), 197-210. 
[41] S. Shelah. Cardinal Arithmetic, volume 29 of Oxford Logic Guides. Oxford University Press, New York, 1994.

[42] R. M. Stephenson, Jr., Initially $\kappa$-Compact and Related Spaces, Handbook of set-theoretic topology, North-Holland, Amsterdam, 1984, 603-632.

[43] M. G. Tkačenko, Chains and cardinals, Soviet Math. Dokl. 19 (1978) 382-385.

[44] V. V. Tkachuk, Spaces that are projective with respect to classes of mappings, Transactions of the Moscow Mathematical Society 50 (1988), 139-156.

[45] J. van Mill, An Introduction to $\beta \omega$, Handbook of set-theoretic topology, North-Holland, Amsterdam, 1984, 503-567. 


\section{Índice Remissivo}

$0^{\sharp}, 5$

$\beta X, 7$

$\beth, 2$

$\chi(x, X), 10$

$\chi(X), 10$

CARD, 1

$|X|, 1$

$c(X), 9$

$c f(\alpha), 1$

cf $\left([\mu]^{\lambda}, \subset\right), 52$

$A \rightarrow x, 24$

$x_{\alpha} \rightarrow x, 24$

$\operatorname{cov}(\mu, \chi, \lambda), 52$

$\operatorname{cov}(\mu, \chi, \lambda, \sigma), 68$

$d(X), 8$

$\chi S(p, X), 25$

$\chi S(X), 25$

$c S(p, X), 25$

$c S(X), 25$

$d c S(p, X), 25$

$d c S(X), 25$

$e(X), 9$

FIX, 4

$F(X), 9$

$\widehat{F}(X)$, veja $\widehat{\phi}(X)$

I, 3

$h \phi, 11$

$H_{\theta}, 16$

Hus $(x, X), 29$

Hus (X), 29

$\kappa^{\lambda}, 2$ $\kappa^{<\lambda}, 2$

$\lambda+\mu, 2$

$\lambda \cdot \mu, 2$

]$\lambda, \mu[, 3$

]$\lambda, \mu], 3$

$[\lambda, \mu[, 3$

$[\lambda, \mu], 3$

$L(X), 9$

$l S(X), 50$

$\overline{l S}(X), 50$

ll $(X), 9$

llS $(X), 63$

$m(C), 50$

$m l(X), 57$

$\models, 15$

ORD, 1

ot $(W), 1$

$\mathcal{P}(X), 1$

$\varphi^{M}, 15$

$\widehat{\phi}(X), 11$

$\pi_{S}, 7$

$\pi_{\delta}, 7$

$\psi(x, X), 10$

$\psi(X), 10$

$\psi_{c}(x, X), 11$

$\psi_{c}(X), 11$

psw $(X), 10$

REG, 1

$s(X), 9$

$\widehat{s}(X)$, veja $\widehat{\phi}(X)$

$M \prec N, 15$ 


$$
\begin{aligned}
& t(x, X), 10 \\
& t(X), 10 \\
& w(X), 8 \\
& X_{M}, 17 \\
& {[X]^{\kappa}, 3} \\
& {[X]^{\leq \kappa}, 3} \\
& {[X]^{<\kappa}, 3}
\end{aligned}
$$

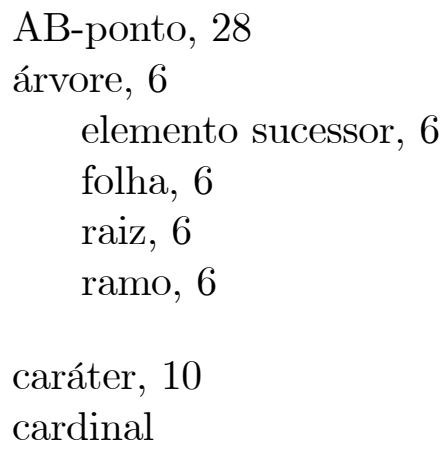

fortemente inacessível, 5 fracamente compacto, 5 fracamente inacessível, 5 limite, 1 limite forte, 1 regular, 1 singular, 1 sucessor, 1

celularidade, 9

$\mathrm{CH}, 5$

cofinalidade, 1

compactificação, 7

de Alexandroff, 7

de Stone-Cech, 7

compactum, 6

AB-compactum, 28

K-compactum, 28

Conjunto de Cantor, 7

convergência

de um subconjunto, 24

de uma sequência, 24

Cubo de Cantor, 7

densidade, 8 espaço

compacto, 6

$d$-separável, 48

de pointwise countable type, 11

diádico, 7

finalmente $\lambda$-compacto, 8

inicialmente $\kappa$-compacto, 8

$\kappa$-Lindelöf, 8

$[\lambda, \kappa]$-compacto, 8

linearmente Lindelöf, 8

separado à direita, 6

separado à esquerda, 6

espectro

de caráter, 25

de convergência, 25

de convergência discreto, 25

extent, 9

freeness, 9

função

beth, 2

gimel, 3

potência, 3

função cardinal, 8

monótona, 11

GCH, 5

grau de Lindelöf, 9

grau de Lindelöf linear, 9

Hipótese

do Contínuo, 5

do Contínuo Generalizada, 5

para Cardinais Singulares, 5

K-ponto, 28

$L(\kappa)$-espaço, 8

peso, 8

point separating weight, 10

ponto de acumulação completo, 6

propriedade de Darboux, 18 
propriedade $I U(\kappa), 17$

pseudocaráter, 10

pseudocaráter fechado, 11

reflexão de funções cardinais, 17

reflexão forte, 17

relativização de uma fórmula, 15

$S C H, 5$

sequência livre, 9

spread, 9

SSH, 60

submodelo elementar, 15

$\kappa$-covering, 16

tightness, 10

tipo de ordem, 1 